NASA/TM-2005-213559

\title{
Realizing "2001: A Space Odyssey": Piloted Spherical Torus Nuclear Fusion Propulsion
}

Craig H. Williams, Leonard A. Dudzinski, Stanley K. Borowski, and Albert J. Juhasz Glenn Research Center, Cleveland, Ohio 
Since its founding, NASA has been dedicated to the advancement of aeronautics and space science. The NASA Scientific and Technical Information (STI) Program Office plays a key part in helping NASA maintain this important role.

The NASA STI Program Office is operated by Langley Research Center, the Lead Center for NASA's scientific and technical information. The NASA STI Program Office provides access to the NASA STI Database, the largest collection of aeronautical and space science STI in the world. The Program Office is also NASA's institutional mechanism for disseminating the results of its research and development activities. These results are published by NASA in the NASA STI Report Series, which includes the following report types:

- $\quad$ TECHNICAL PUBLICATION. Reports of completed research or a major significant phase of research that present the results of NASA programs and include extensive data or theoretical analysis. Includes compilations of significant scientific and technical data and information deemed to be of continuing reference value. NASA's counterpart of peerreviewed formal professional papers but has less stringent limitations on manuscript length and extent of graphic presentations.

- TECHNICAL MEMORANDUM. Scientific and technical findings that are preliminary or of specialized interest, e.g., quick release reports, working papers, and bibliographies that contain minimal annotation. Does not contain extensive analysis.

- CONTRACTOR REPORT. Scientific and technical findings by NASA-sponsored contractors and grantees.
- CONFERENCE PUBLICATION. Collected papers from scientific and technical conferences, symposia, seminars, or other meetings sponsored or cosponsored by NASA.

- SPECIAL PUBLICATION. Scientific, technical, or historical information from NASA programs, projects, and missions, often concerned with subjects having substantial public interest.

- TECHNICAL TRANSLATION. Englishlanguage translations of foreign scientific and technical material pertinent to NASA's mission.

Specialized services that complement the STI Program Office's diverse offerings include creating custom thesauri, building customized databases, organizing and publishing research results ... even providing videos.

For more information about the NASA STI Program Office, see the following:

- Access the NASA STI Program Home Page at http://www.sti.nasa.gov

- E-mail your question via the Internet to help@sti.nasa.gov

- Fax your question to the NASA Access Help Desk at 301-621-0134

- Telephone the NASA Access Help Desk at 301-621-0390

- Write to:

NASA Access Help Desk

NASA Center for AeroSpace Information 7121 Standard Drive

Hanover, MD 21076 
NASA/TM-2005-213559

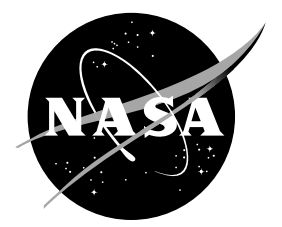

\section{Realizing "2001: A Space Odyssey": Piloted Spherical Torus Nuclear Fusion Propulsion}

Craig H. Williams, Leonard A. Dudzinski, Stanley K. Borowski, and Albert J. Juhasz Glenn Research Center, Cleveland, Ohio

Prepared for the

37th Joint Propulsion Conference and Exhibit

cosponsored by the AIAA, SAE, AIChE, and ASME

Salt Lake City, Utah, July 8-11, 2001

National Aeronautics and

Space Administration

Glenn Research Center 


\section{Acknowledgments}

The authors wish to thank many who have been very helpful in providing guidance and expertise: Ben Clark, Lockheed Martin Corporation, in the area of artificial gravity payload design; John Santarius, University of Wisconsin, in the areas of synchrotron radiation management and first wall design; Martin Peng, Princeton Plasma Physics Lab (PPPL), in the areas of bootstrap current overdrive, Coaxial Helicity Ejection, and high harmonic fast wave heating; David Dombrowski, Brush Wellman, Inc., Michael Ulrickson, Sandia National Lab, and Glen Longhurst, Idaho National Engineering Lab, in the area of fusion applications of beryllium alloys; Dave Plachta, NASA Glenn Research Center (NASA Glenn) and Peter Kittel, NASA Ames Research Center, in the area of spacequalified cryogenic refrigeration; Gerald Hale, Los Alamos National Lab (LANL), in the area of spin polarized $\mathrm{D}^{3} \mathrm{He}$ fuel; Stanley Kaye, PPPL, in the area of fusion confinement time scaling laws; Joseph Warner, NASA Glenn, in the area of YBCO superconductor material properties and prospects; Ronald Moses, LANL, in the area of D-shape magnet designs and forces; Lawrence Green, Westinghouse Science and Technology Center and Daniel Driemeyer, Boeing Corporation, in the area of ITER divertor design drivers; Peter Turchi and Hani Kamhawi, Ohio State University, in the area of magnetic nozzle design and state conditions; Richard Gerwin, LANL, in the area of theory development characterizing plasma resistivity; Tom Goodnight, NASA Glenn, in the area of structural loading; Joe Roche, NASA Glenn, in the area of carbon-graphite composite material properties; David Swain, Oak Ridge National Lab (ORNL), in the area of RF heating mass properties; Richard Kunath, NASA Glenn, in the area of Ka band space communications; Robert Cataldo, NASA Glenn, in the area of start-up NiH batteries; John Sankovic, NASA Glenn, and R. Joseph Cassady, Primex Technologies Corporation, in the area of high power hydrogen arcjets; Mellissa McGuire, Analex Corporation, in the area of reaction control; Jim Dolce, NASA Glenn, in the area of startup torque/momentum control flywheels; Judith Watson, NASA Langley Research Center, in the area of Space Station truss network; and Mohamed Bourham, North Carolina State University, and Stanley Milora, ORNL, in the area of fuel injectors.

\footnotetext{
Note that at the time of writing, the NASA Lewis Research Center was undergoing a name change to the

NASA John H. Glenn Research Center at Lewis Field. Both names may appear in this report.
}

Available from

NASA Center for Aerospace Information 7121 Standard Drive

Hanover, MD 21076
National Technical Information Service 5285 Port Royal Road Springfield, VA 22100

Available electronically at http:/ /gltrs.grc.nasa.gov 


\title{
REALIZING "2001: A SPACE ODYSSEY": PILOTED SPHERICAL TORUS NUCLEAR FUSION PROPULSION
}

\author{
Craig H. Williams, Leonard A. Dudzinski, Stanley K. Borowski, and Albert J. Juhasz \\ National Aeronautics and Space Administration \\ Glenn Research Center \\ Cleveland, Ohio 44135
}

\begin{abstract}
A conceptual vehicle design enabling fast, piloted outer solar system travel was created predicated on a small aspect ratio spherical torus nuclear fusion reactor. The initial requirements were satisfied by the vehicle concept, which could deliver a $172 \mathrm{mt}$ crew payload from Earth to Jupiter rendezvous in 118 days, with an initial mass in low Earth orbit of 1,690 mt. Engineering conceptual design, analysis, and assessment was performed on all major systems including artificial gravity payload, central truss, nuclear fusion reactor, power conversion, magnetic nozzle, fast wave plasma heating, tankage, fuel pellet injector, startup/re-start fission reactor and battery bank, refrigeration, reaction control, communications, mission design, and space operations. Detailed fusion reactor design included analysis of plasma characteristics, power balance/utilization, first wall, toroidal field coils, heat transfer, and neutron/x-ray radiation. Technical comparisons are made between the vehicle concept and the interplanetary spacecraft depicted in the motion picture 2001: A Space Odyssey.
\end{abstract}

\section{INTRODUCTION}

The impetus for this effort was three fold. First: to guide the long range National Aeronautics and Space Administration (NASA) goal of human expansion throughout the solar system, a rational approach for long term research and development must be clearly articulated. Second: currently funded nuclear fusion space propulsion research must be on the critical path for enabling order-of-magnitude improvements in future space transportation capability. Third: a conceptual vehicle design incorporating the proposed design philosophies and related results of a recent series of NASA Glenn (formerly Lewis) Research Center (GRC) papers was the next step in the process. ${ }^{1,2,3,4,5,6}$
The findings of these earlier papers emphasized that for piloted, outer solar system missions expected within the $21^{\text {st }}$ century, adequate payload mass fraction (5\% to $15 \%)$ and multi-month trip times would require specific impulses $\left(\mathrm{I}_{\mathrm{sp}}\right)$ and specific powers $(\alpha)$ of 20,000 to $50,000 \mathrm{lb}_{\mathrm{f}} \mathrm{sec} / \mathrm{bb}_{\mathrm{m}}$ and 5 to $50 \mathrm{~kW} / \mathrm{kg}$ respectively ${ }^{1,4,5,6}$. It is the judgment of the authors that direct nuclear fusion space propulsion is the leading technology that can reasonably be expected to offer this capability.

Nuclear fusion reactors can be broadly classified into at least three groups: closed magnetic (such as tokamaks, small aspect ratio toroids, spheromaks, field reversed, etc.), open magnetic (mirrors), and inertial concepts. Based in part on the results of previous studies ${ }^{4,7}$ of the attributes and shortcomings of these reactor groups towards space propulsion, a closed magnetic system was chosen for this vehicle concept. The high power density achievable in closed systems, improved confinement, spin polarization of fuel, density and temperature profile peaking provided advantages in application towards space propulsion. While large aspect ratio tokamaks have been the predominant focus of the fusion research community for many years, their great size and mass render them unappealing for space propulsion, where lightweight is paramount. At the other end of the closed reactor spectrum, spheromak and field reversed concepts offer tremendous hope for compact, lightweight propulsion-oriented systems. However, the dearth of experimental data on their operation and indeed their engineering feasibility render serious engineering assessment difficult. As a result, a compromise was struck between existing, extrapolatable experimental databases from tokamaks and the largely conceptual compact toroids. The small aspect ratio spherical torus, a concept midway between these two groups, was thus chosen to serve as the basis for the vehicle concept. 
Consistent with the "top-down" requirementsdriven approach documented elsewhere, the vehicle design was initiated by first establishing a simple set of mission requirements, then producing a consistent engineering design that satisfied those requirements. This meant that current state of the art systems, along with experimental results, were used as the basis for extrapolation to what could be technologically available to a human presence solar system-wide of the not too distant future-30 years from now. All system engineering analysis was performed using existing computer programs, open literature engineering sources, and basic engineering calculations. The preponderance of the nuclear fusion engineering data was obtained from U.S. Department of Energy's (DOE) terrestrial power and scientific research programs, while much of the propulsion system engineering data was derived from NASA expendable launch vehicle and conceptual nuclear thermal rocket (NTR) design studies. Only limited NASA fusion space propulsion system data exists beyond what was accomplished since the termination of the 20 year nuclear fusion program at NASA GRC (LeRC) in 1978.

\section{1: A SPACE ODYSSEY}

At the end of this paper, a brief discussion is included on a topic outside the norm. It is worthy to note in this year of 2001, the similarities between the spacecraft in that highly acclaimed motion picture epic and best selling novel 2001: A Space Odyssey and this NASA vehicle concept. That landmark film by the celebrated director Stanley Kubrick and the legendary science fiction author Arthur C. Clarke was perhaps the most thoroughly and accurately researched film in screen history with respect to aerospace engineering. The technology depicted was extracted from numerous NASA and industry engineers and their design studies. Dr. Frederick Ordway, staff to Dr. Wernher Von Braun, was the primary NASA consultant to the film. Much of his team's expertise went into the technical accuracy implicit in the propulsion systems depicted in the film. The mission of 2001's Discovery spaceship, an Earth to Jupiter rendezvous (in the novel: Earth to Saturn), was chosen as the design reference mission for this vehicle concept. To realize 2001's dream, a vehicle similar to what is described here will be necessary. In addition to furthering NASA's long range goals, this year, this conceptual design is also a modest attempt to honor the technical excellence and enlightened leadership that so characterized that generation of NASA leaders and visionaries of the Arts. We have therefore christened this NASA vehicle concept: the Discovery II.

\section{MISSION REQUIREMENTS}

The top-down, mission requirements-driven design process began with specifying the desired mission, trip time, and payload mass fraction. These requirements were then used to define operation parameters: mission distance, specific power, specific impulse, and nozzle jet efficiency. These operation parameters are directly related to four primary system characteristics: structure mass, power out of reactor, jet power, and thrust. The primary system characteristics focused the engineering design effort and were iterated on until mission requirements were satisfied.

The reference missions selected were to destinations in the outer solar system, where dozens of scientifically interesting worlds will compel human exploration in the future. The missions were to provide a logical progression to the considerable mission planning NASA has conducted over the years to more near term, inner solar system destinations (i.e. the Moon, Mars). Outer solar system distances are $\sim 1$ to 2 orders of magnitude greater than those in the inner solar system and thus more likely to require revolutionary improvements in propulsion. The design reference mission was a Jupiter rendezvous, with a more demanding alternate mission to Saturn.

The Discovery II was to be able to perform a rendezvous (one way) mission to either Jupiter or Saturn, piloted by a crew of six to twelve, with a $>5 \%$ payload mass fraction, and a trip time of less than one year. The specific destinations were Jupiter's moon Europa and Saturn's moon Titan. These two moons were selected because of their: demanding performance requirements, scientifically interesting possibility of life on their surfaces requiring human presence for investigation, dominant size among other moons, expected abundance of hydrogen for propulsion application, abundance of fusion fuels $\mathrm{D}_{2}$ and ${ }^{3} \mathrm{He}$ in their planet's atmosphere. Based primarily on existing humans to Mars mission studies, a minimum crew size of six was chosen. The piloted nature of the mission also drove the requirement for relatively fast trip times. The one-year maximum was somewhat arbitrary, but was representative of long duration human experience in low Earth orbit and consistent with some current Mars mission studies. The intent was to force the Discovery II to perform multi-month (vs. multi-year) missions, despite interplanetary distances that dwarf the more commonly thought of Earth to Mars transfers. The mission distance was predicated on optimal planetary orientation, though performance requirements for more demanding planetary positions were also evaluated. 


\section{VEHICLE OVERVIEW}

Figures 1, 2, and 3 illustrate the overall layout of the Discovery II. The rotating crew payload was forward of the propulsion system. It was linked to the central truss through a fixed central hub, which also attached to the avionics suite and truss booms supporting the communication antennas. The forward central truss supported the two, co-planar, low and high temperature heat rejecting radiators. Along the outside of the mid-central truss were four slush hydrogen propellant tanks. Within the mid-central truss was the $\mathrm{D}^{3} \mathrm{He}$ fuel tank and refrigeration system for all propellant/fuel tankage. Throughout the central truss were also various data, power, coolant, and propellant lines. Within the aft central truss was the Brayton power conversion system. Also within the aft central truss were the power management and distribution system, the refrigeration system, the start/re-start reactor and battery bank. Running the entire length of the central truss was the fuel pellet injection system. Aft of the central truss were the spherical torus nuclear fusion reactor, fast wave heating, and the magnetic nozzle. The overall vehicle length was $240 \mathrm{~m}$. The longest deployed system dimensions were the $203 \mathrm{~m}$ central truss and the $25 \mathrm{~m}$ heat rejection (radiator) systems. The maximum stowed diameter for any individual system, however, was limited to $10 \mathrm{~m}$ so as to fit within the envisioned payload fairing, facilitating launch and on-orbit assembly. The fully tanked initial mass in low Earth orbit (IMLEO) was 1,690 mt.
Table 1: Vehicle Mass Property Summary

\begin{tabular}{|c|c|c|c|}
\hline \multirow[t]{5}{*}{ Payload } & & & 172 \\
\hline & Structure & 38 & \\
\hline & Shielding & 69 & \\
\hline & Crew systems & 25 & \\
\hline & Weight growth contingency & 40 & \\
\hline \multirow[t]{15}{*}{ Structure } & & & 646 \\
\hline & Central truss & 6 & \\
\hline & Fusion reactor & 310 & \\
\hline & Magnetic nozzle and divertor & 6 & \\
\hline & Reaction control & 16 & \\
\hline & Power conversion & 30 & \\
\hline & Coolant system & 11 & \\
\hline & Fast wave plasma heating & 5 & \\
\hline & Propellant cryo-tankage & 88 & \\
\hline & Refrigeration & 2 & \\
\hline & Fuel tankage and injector & 6 & \\
\hline & Startup/re-start fission reactor & 10 & \\
\hline & Battery bank & 5 & \\
\hline & Avionics and communication & 2 & \\
\hline & Weight growth contingency & 149 & \\
\hline $\mathrm{D}^{3} \mathrm{He}$ fuel & & & 1 \\
\hline \multirow[t]{5}{*}{ Hydrogen } & propellant & & 861 \\
\hline & Main impulse & 807 & \\
\hline & Reaction control & 20 & \\
\hline & Flight performance reserve & 8 & \\
\hline & Residuals/losses & 26 & \\
\hline IMLEO & $(\mathrm{mt})$ & & 1,69 \\
\hline
\end{tabular}

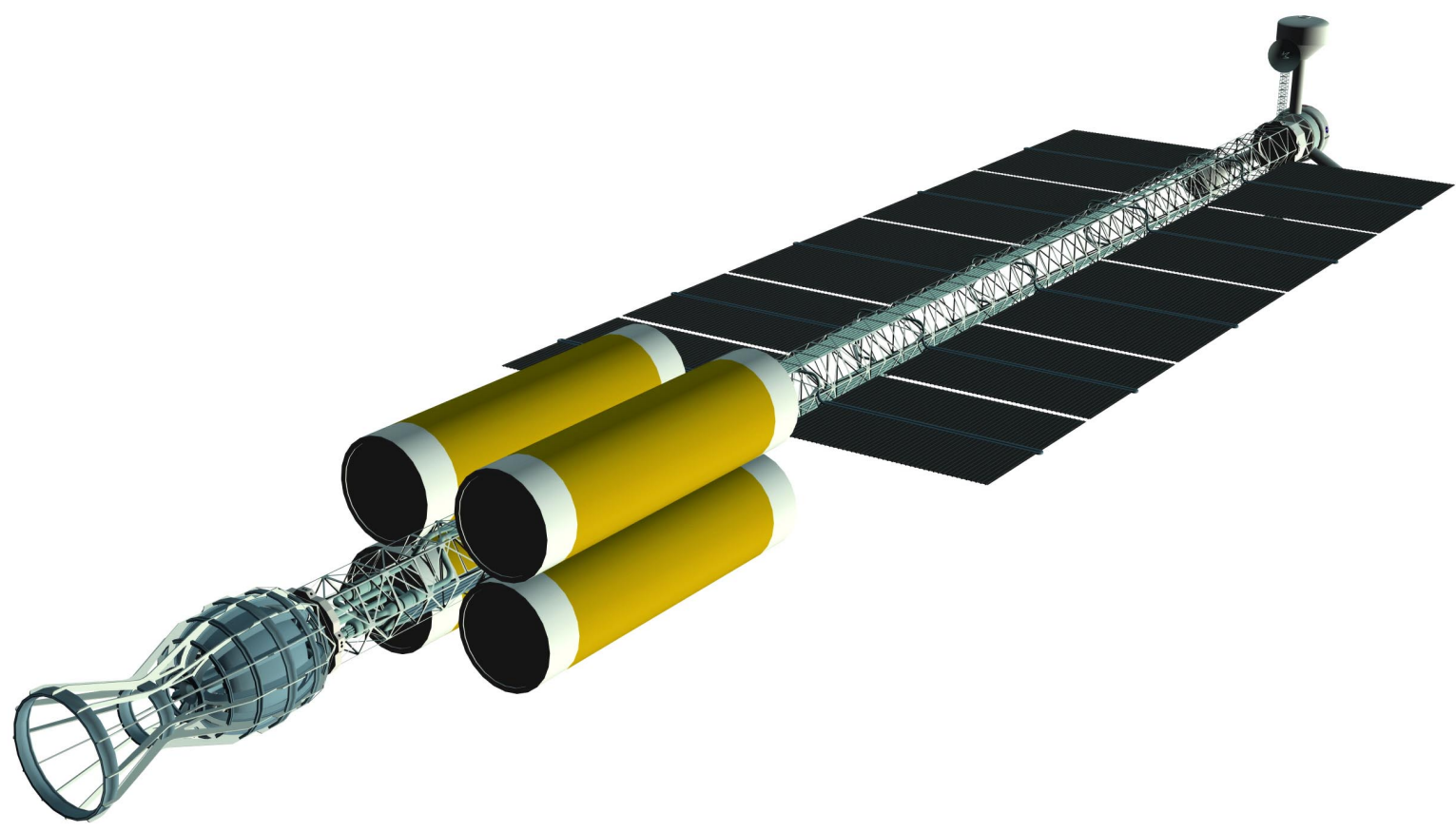

Figure 1: The Discovery II (aft view) 


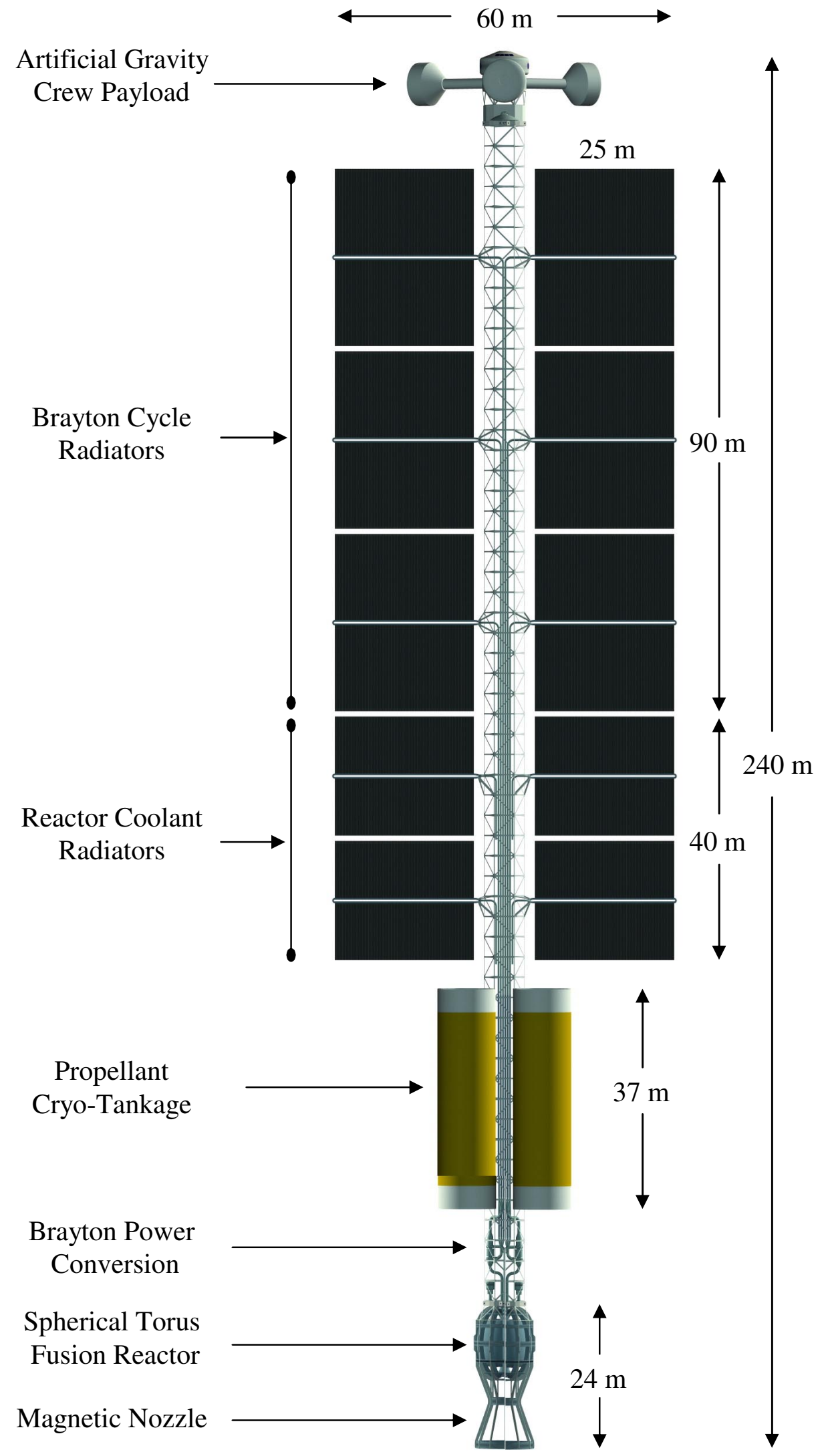

Figure 2: The Discovery II (top view) 


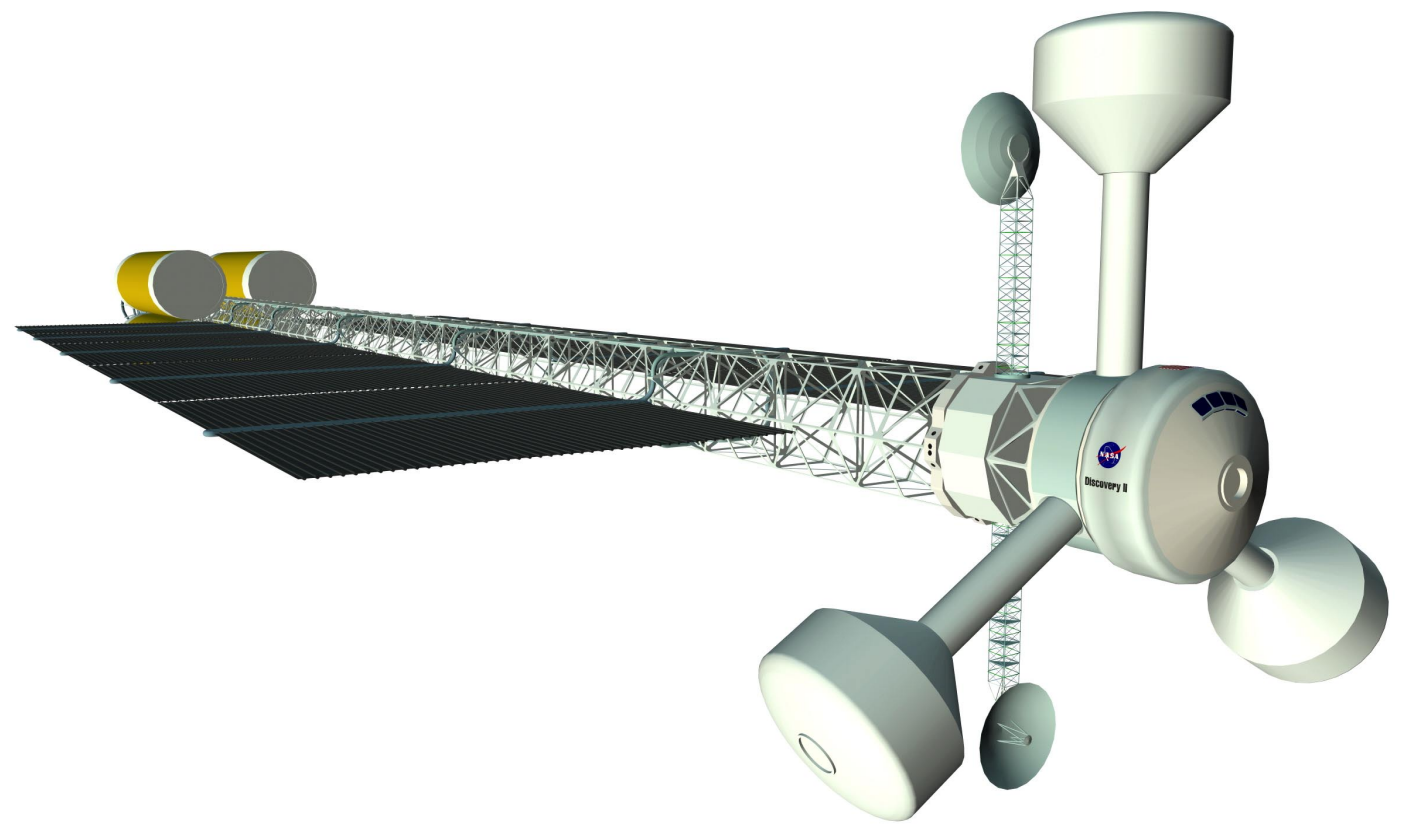

Figure 3: The Discovery II (front view)

The design philosophy followed was to locate as much of the vehicle mass (power conversion, reactor, and propellant) as close to and forward of the thrust vector as possible to facilitate steering control authority. Although shielded and emitting only laterally directed radiation, the reactor was kept as far aft of the crew payload as possible. The modular vehicle layout, system packaging, launch operations, and in-space assembly sequence was expected to be conducted in a way that maximized docking maneuvers rather than labor intensive Extra Vehicular Activity (EVA). Since the vehicle was designed for interplanetary cruise, only a docking port was provided to accommodate surfacebased landing craft.

Table 1 illustrates the mass property summary for the design reference mission's fully loaded stack. The "payload mass" was $172 \mathrm{mt}$ and consisted of useful payload only (crew, habitat, consumables, shielding, weight growth contingency, etc.) The "fuel" was $11 \mathrm{mt}$ of $\mathrm{D}^{3} \mathrm{He}$ for the nuclear fusion reactor. The slush hydrogen "propellant mass" was $861 \mathrm{mt}$ and was used for main impulse, reaction control, reserves, and losses. It did not include system or tankage mass. (For the purposes of calculating the velocity ratio only, the propellant mass $\left(\mathrm{M}_{\mathrm{p}}\right)$ was taken to be the main impulse propellant and fuel. The balance of slush hydrogen (reserves, etc.) was book-kept with the structure mass.) The total structure mass $\left(\mathrm{M}_{\mathrm{s}}\right)$ was $646 \mathrm{mt}$ and referred to all mass required to operate the propulsion system, including weight growth contingency.

\section{MISSION ANALYSIS}

Fusion propulsion systems are expected to operate at high enough $I_{\mathrm{sp}}$ and $\alpha$ to produce accelerations greater than the local acceleration due to solar gravity at Earth's orbit (0.6 milli-g; where 1 milli-g = $\left.32.173910^{-3} \mathrm{ft} / \mathrm{sec}^{2}\right){ }^{1,4}$ The normally thought of conics of minimum energy trajectories followed by today's chemical systems degenerate into nearly straight line, radial transfers at these high acceleration levels with continuous thrust. A "field-free space" approximation can be invoked to greatly simplify the usually complex orbital mechanics. Gravity losses and optimum steering concerns can be neglected without introducing too much error, obviating the need for computationally intensive, numerically integrated solutions to support preliminary analysis. In addition, a "launch at anytime" approach to mission design is a luxury that can usually be assumed for fusion systems so long as the thrust to weight is great enough compared to the local acceleration due to solar gravity. As will be shown, despite an initial thrust to weight of only 1.68 milli-g, the Discovery II's trajectory was reasonably close to that of a radial transfer.

At the initiation of and throughout the design effort, a simple high $\mathrm{I}_{\mathrm{sp}} /$ high thrust algorithm $^{6}$ for radial transfers was used to initiate and guide the design process and monitor convergence towards study requirements. This analytic, closed-form solution previously published by the co-author was used for the initial trip time and performance analysis. The governing relations were based on the classic rocket 


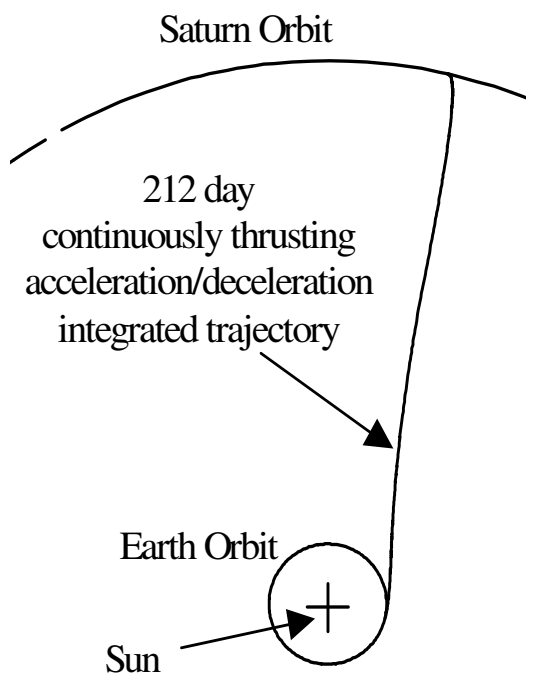

Figure 4: Integrated Earth to Saturn Trajectory (Alternate Mission)

equation and auxiliary relations for high (constant) thrust constant mass flow rate (variable acceleration) travel through field-free space.

At the conclusion of the design effort, the high fidelity, variational calculus-based trajectory optimization program VARITOP was used to verify vehicle performance and overall mission design. VARITOP is a two body, heliocentric transfer computer program for modeling low thrust space propulsion systems. ${ }^{8}$ It is well known throughout the preliminary design community for its good accuracy in solving the two point boundary value problem and integrating state variables. Good agreement was found between the two trajectory design computer programs. Figure 4 illustrates a heliocentric view of the integrated VARITOP trajectory from Earth to Saturn.

A few definitions of operational characteristics and system parameters are as follows. Specific power $(\alpha)$ is calculated differently throughout the space propulsion community. Here we will use the common, though perhaps not universal, definition of the ratio of power out from the reactor system $\left(\mathrm{P}_{\text {out }}\right)$ (and sent into the thrust generating device) divided by $\mathrm{M}_{\mathrm{s}}$ (including reaction control propellant, reserves, and residuals) (Equation (1)). The $\alpha$ for the concept was $8.62 \mathrm{~kW} / \mathrm{kg}$.

$$
\alpha \equiv \frac{P_{o u t}}{M_{s}}
$$

The nozzle jet efficiency $\left(\eta_{\mathrm{j}}\right)$ is the effectiveness of converting transport power out of the reactor into directed jet power in the thrust exhaust as defined in Equation (2). The $\eta_{J}$ for this class of systems remains largely conjecture, consequently a value of 0.8 (i.e. $80 \%$ ) was assumed based on known low power electric propulsion systems and analytically derived minimum fusion system efficiencies ${ }^{9,10}$.

$$
\eta_{J}=\frac{P_{\text {jet }}}{P_{\text {out }}}
$$

Using these definitions and constant total mass flow rate (m-dot $t_{\text {total }}$, the total flow rate of propellant and reactor fuel and $\left.\mathrm{g}_{\mathrm{c}}=32.1739 \mathrm{lb}_{\mathrm{m}} \mathrm{ft} /\left(\mathrm{lb}_{\mathrm{f}} \sec ^{2}\right)\right)$, the jet thrust power $\left(\mathrm{P}_{\mathrm{jet}}\right)$, thrust $(\mathrm{F}), \mathrm{I}_{\mathrm{sp}}$, and exhaust velocity (c) can all be solved for using the familiar Equation (3):

$$
P_{\text {jet }}=\eta_{J} P_{\text {out }}=\frac{F g_{c} I_{s p}}{2}=\frac{F^{2} g_{c}}{2 \dot{m_{\text {total }}}}=\frac{\dot{m_{\text {total }} c^{2}}}{2 g_{c}}
$$

Table 2 contains the overall performance analysis results for the Discovery II. All vehicle mass properties were fixed for both missions (with only a minor adjustment for fuel loading). In addition, other properties were fixed: $P_{\text {out }}, \eta_{\mathrm{J}}, \alpha$, and velocity ratio. The $\mathrm{I}_{\mathrm{sp}}, \mathrm{F}$, and $\mathrm{m}$-dot $\mathrm{dtat}_{\text {tal }}$ were mission peculiar. The Earthto-Jupiter rendezvous mission thrust was $6,250 \mathrm{lb}_{\mathrm{f}}, \mathrm{I}_{\mathrm{sp}}$ of $35,435 \mathrm{lb}_{\mathrm{f}} \mathrm{sec} / \mathrm{lb}_{\mathrm{m}}$, and had a propellant flow rate of $0.079 \mathrm{~kg} / \mathrm{sec}$. The Earth-to-Saturn rendezvous mission thrust was $4,690 \mathrm{lb}_{\mathrm{f}}, \mathrm{I}_{\mathrm{sp}}$ of $47,205 \mathrm{lb}_{\mathrm{f}} \mathrm{sec} / \mathrm{lb}_{\mathrm{m}}$, and had a propellant flow rate of $0.044 \mathrm{~kg} / \mathrm{sec}$. Rendezvous missions were integrated for the optimal departure dates. The payload modules for the Jupiter and Saturn missions were both $172 \mathrm{mt}$. The 118 day ( 4 month) trip time to Jupiter and 212 day ( $\sim 7$ month) trip time to Saturn were rapid compared to those of representative alternate concepts, where similar rendezvous mission trip times using chemical or even nuclear thermal propulsion would be measured in years. An analytic approximation was made of the trip time for opposition orientation of the planets. The Earth-to-Saturn trip time was lengthened by only $15 \%$, illustrating the relative insensitivity to launch date due to the high thrust to weight capability. These results suggest that the Discovery II could accomplish fast interplanetary trip times with significant payloads over broad launch opportunities. Further improvements in payload and trip time could be achieved if fusion technology advances permitted optimal propellant mass loadings. 
Table 2: Performance Analysis Results

\begin{tabular}{lcc}
\hline Destination & $\begin{array}{c}\text { Jupiter } \\
\text { Rendezvous }\end{array}$ & $\begin{array}{c}\text { Saturn } \\
\text { Rendezvous }\end{array}$ \\
Trassion type & 4.70 & 9.57 \\
Specific power $(\mathrm{kW} / \mathrm{kg})$ & 8.62 & $\sim$ same \\
Specific impulse $\left(\mathrm{lb}_{\mathrm{f}} \mathrm{sec} / \mathrm{lb}_{\mathrm{m}}\right)$ & 35,435 & 47,205 \\
Payload mass $(\mathrm{mt})$ & 172 & same \\
IMLEO (mt) & 1,690 & 1,699 \\
Trip time $(\mathrm{days})$ & 118 & 212 \\
Jet power $(\mathrm{MW})$ & 4,830 & same \\
Jet efficiency & 0.8 & same \\
Thrust $\left(\mathrm{lb}_{\mathrm{f}}\right)$ & 6,250 & 4,690 \\
Total flow rate $(\mathrm{kg} / \mathrm{sec})$ & 0.080 & 0.045 \\
Exhaust velo/char velo & 0.92 & $\sim$ same \\
Initial thrust/mass (milli-g) & 1.68 & 1.25 \\
\hline
\end{tabular}

The Discovery II was designed to carry a suboptimal payload mass with a non-optimal propellant loading, contrary to earlier study recommendations. ${ }^{4}$ Nuclear fusion technology offers perhaps the greatest useful power out for high thrust/high $I_{\text {sp }}$ propulsion technology appropriate for interplanetary mission requirements. But fusion's range of $\alpha$ 's for credible propulsion system designs (coupled to large $\mathrm{M}_{\mathrm{s}}$ ) are still generally too low for optimum IMLEO's of less than $1,000 \mathrm{mt}$. Simply put, most projected fusion propulsion systems remain massive despite the large jet power they produce. The ratio of exhaust to characteristic velocities $\left(\mathrm{V}_{\mathrm{c}}\right)\left(\mathrm{c} / \mathrm{V}_{\mathrm{c}}\right.$, a convenient measure of optimal propellant loading with respect to $\mathrm{M}_{\mathrm{s}}$ ) vs. payload mass fractions for representative mission difficulty factors $\left(\Delta \mathrm{V} / \mathrm{V}_{\mathrm{c}}\right)$ (where velocity increment $(\Delta \mathrm{V})$ ) are illustrated in Figure 5, with the Discovery II operating point also

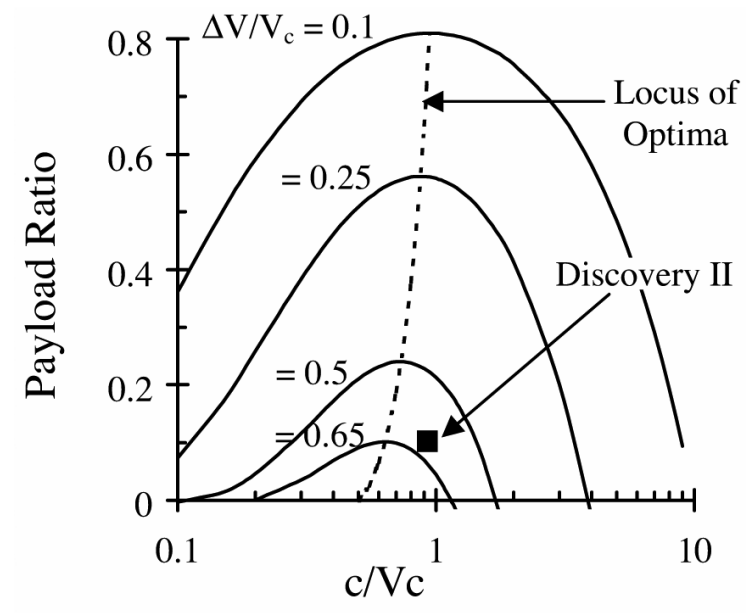

Figure 5: Payload Mass Fraction vs. Velocity Ratio plotted. It shows that the Discovery II (with mission difficulty $\Delta \mathrm{V} / \mathrm{V}_{\mathrm{c}}=0.61$ ) was not optimally loaded with propellant and considerably removed from the maximum potential payload fraction. An optimal propellant loading for the same propulsion system mass and $\Delta \mathrm{V} / \mathrm{V}_{\mathrm{c}}$ would have been twice the design amount (i.e. eight cryo-tanks). Alternatively, fewer than four propellant cryo-tanks would have further increased $\mathrm{c} / \mathrm{V}_{\mathrm{c}}$, while decreasing payload ratio, lengthening trip time, and driving the concept further from the optimal value of $\mathrm{c} / \mathrm{V}_{\mathrm{c}}=0.66$. Thus a four cryo-tank configuration was chosen, yielding a non-optimal, but reasonable $\mathrm{c} / \mathrm{V}_{\mathrm{c}}=0.92$.

\section{SPACE OPERATIONS}

Space operations issues pertain to IMLEOdriven Earth to orbit (ETO) launch, assembly, departure/arrival park orbit basing, and rendezvous vs. round trip propellant loading modus operandi. These issues are interrelated and have a profound influence on total system viability.

The large $\mathrm{M}_{\mathrm{s}}$-driven IMLEO represented a fundamental obstacle to viable space operations. The Discovery II's IMLEO was $\sim 17$ times what could be delivered to LEO by a launch system in the Space Shuttle-class (that is: $80 \mathrm{mt}$ (orbiter) $+22 \mathrm{mt}$ (payload) $=102 \mathrm{mt}$ to a $140 \mathrm{nmi}$ circular orbit inclined $\left.28.5^{\circ}\right) .^{11}$ This could be accomplished with the so-called "ShuttleC" booster, a long proposed derivative of the existing system that would operate without the Shuttle orbiter. More likely, a new heavy lift launch vehicle (HLLV) would be required. Sized for the greatest single payload masses and volumes, the HLLV payload capability could be as great as $250 \mathrm{mt}$ to LEO. This throw-weight capability of almost 2 "equivalent three stage Saturn V's", was at the upper range of past design studies ${ }^{12}$ and would be required to deliver a single, fully loaded propellant tank to LEO. Even with this class of launch vehicle, 7 would be required for the initial mission, and 4 for subsequent missions (propellant re-supply).

The first launch would deliver the central truss, auxiliary power system (nuclear fission reactor), reaction control system (with limited propellant), battery bank, communications, avionics, power conversion, main radiators, refrigeration, PF coils, fully loaded fuel tankage, injector, and other systems. After its deployment, auxiliary power on a stable truss with computer command/control would be in orbit to enable rendezvous by subsequent HLLV's. The second launch would deliver the fusion reactor and magnetic nozzle. The third launch would deliver the entire artificial gravity payload. Launches \#4 through \#7 would be fully loaded propellant tankage. A preliminary manifest was 
prepared to ensure that each HLLV launch could accommodate the mass and dimensions of aggregate payloads. Table 3 illustrates assembly characteristics.

Table 3: Assembly Characteristics

\begin{tabular}{lc}
\hline $\begin{array}{c}\text { Number of HLLV launches } \\
\text { Vehicle }\end{array}$ & 3 \\
Propellants & 4 \\
Assembly sequence & \\
Flight 1 & truss, aux power, RCS, etc. \\
Flight 2 & fusion reactor, nozzle, etc. \\
Flight 3 & payload, etc. \\
Flights 4, 5, 6, & propellant \\
HLLV throw weight (mt) & 251 \\
HLLV payload fairing & 10 \\
Diameter (m) & 37 \\
Length (m) & \\
Assembly orbit & 260 circular \\
Altitude (nmi) & 28.5 \\
Inclination (deg) & Rendezvous, dock \\
Assembly method & dedicated vehicle \\
Crew transport & 1,690 \\
IMLEO (mt) &
\end{tabular}

On-orbit assembly would be a necessity for this concept. Individual systems would be configured to maximize simpler rendezvous and docking techniques as opposed to telerobotics or labor intensive EVA. A significant amount of on-orbit operations will still nonetheless require human presence despite the significant complexity, cost, and human factor issues surrounding EVA. Major systems (payload, central truss, heat rejection, etc.) would be assembled/deployed in orbit sequentially, facilitating these operations rather than attempting to minimize gross vehicle size by maximizing final vehicle configuration packing density. Assembly orbit altitude will most likely be no higher than $260 \mathrm{nmi}$ due to human radiation exposure limits and HLLV performance limits, while minimum orbits much below $140 \mathrm{nmi}$ are unlikely due to long term atmospheric drag and monatomic oxygen effects. Expensive launch and operations, coupled with expensive fusion technology, will mandate the design requirement for long life and re-use. If fusion concepts actually prove to be as massive as the current design suggests, limits on on-orbit assembly, launch availability, and maximum practical HLLV performance and volume capability could prove development-lethal for fusion propulsion.

Even with a new HLLV, a serious viability issue would still exist regarding launch availability. The ability to launch seven HLLV's within a reasonable time period (months) will remain a dubious proposition for the foreseeable future. And launch costs associated with that many HLLV's would represent a significant percentage of total mission costs. Thus, dramatic reductions in launch processing and increases in robustness in launch availability would be mandatory for such a launch campaign to be viable.

Rendezvous missions were selected as the modus operandi due to their enabling of dramatic reductions in propellant requirements, vehicle size, and improved performance compared to carrying sufficient propellant for round trip missions. ${ }^{1,6}$ The implied requirement of a planetary refueling capability is of great concern, but is consistent with a solar system-class transportation system regularly journeying to and between large outer planets with atmospheres and moons rich in $\mathrm{H}_{2}, \mathrm{D}_{2}$, and ${ }^{3} \mathrm{He}$. Sources of available propellant near high departure/arrival orbits, such as water ice at the lunar poles, minor moons, outermost major moons, and even asteroids, would greatly facilitate refueling without entering into deep gravity wells, provided the facilities could be established and maintained at a sufficiently low cost. It is reasonable to assume that in the time frame of fusion propulsion systems, other technologies and infrastructure (such as semi-robotic mining encampments) would be available. $\mathrm{D}^{3} \mathrm{He}$ fuel would be acquired by either collecting solar wind deposited or scavenging in situ major planetary atmospheric deposits (if cost effective), which would alleviate the ${ }^{3} \mathrm{He}$ supply issue. Processing $40 \mathrm{~km}^{2}$ of lunar regolith to a depth of $2 \mathrm{~m}$, for example, could yield 1 and $6,100 \mathrm{mt}$ of ${ }^{3} \mathrm{He}$ and $\mathrm{H}_{2}$ respectively ${ }^{13}$. If these high orbit basing facilities were not available (or prove too expensive) and all propellant had to be supplied from terrestrial sources for entire round trip missions, then operations could become overwhelming. Little consideration was given at this time to Earthsupplied propellants and fuel after initial assembly due to the perceived greater complexity of operations.

\section{Table 4: Space Operation Characteristics}

\begin{tabular}{lc}
\hline Mission type & rendezvous \\
Maximum range (AU) & $\sim 50$ \\
Maximum duration (months) & 12 \\
Departure/arrival planetary orbits (C3) & $\sim 0$ \\
Infrastructure required per planet & \\
$\quad$ Crew transport (low orbit to C3 0) & 1 \\
$\quad$ Propellant/deuterium ISRU plant & 1 \\
$\quad$ Propellant tankage (autonomous) & 4 \\
$\quad$ Atmospheric miner ( $\left.{ }^{3} \mathrm{He}\right)$ & 1 \\
Number of propellant re-supply launches & 4 \\
Propellant/deuterium ISRU source & major moon \\
Vehicle staging or drop tanks & no \\
Vehicle life & reusable \\
\hline
\end{tabular}




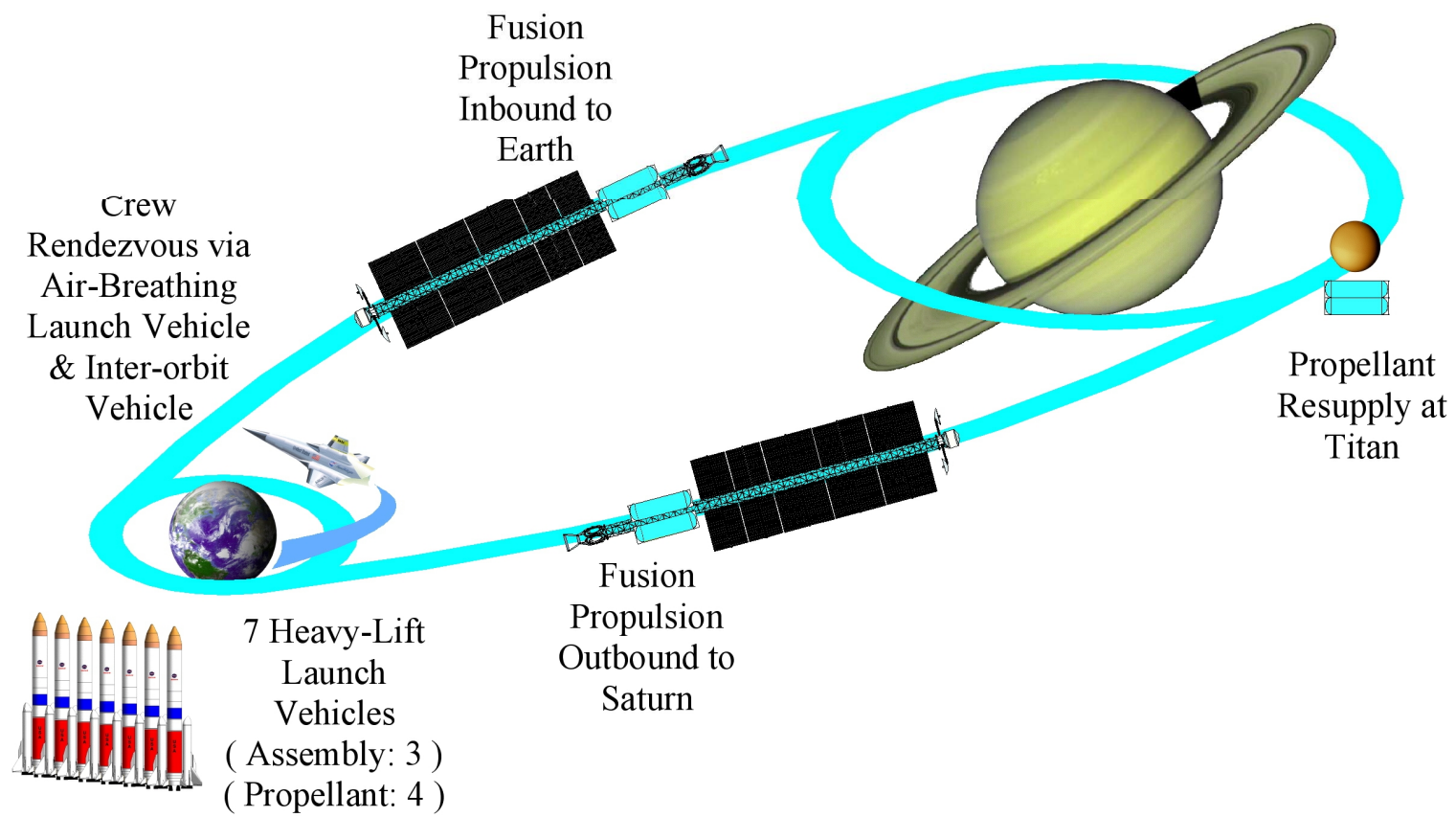

Figure 6: Space Operations Scenario (Alternate Mission)

The Discovery II was thus designed for interplanetary cruise only. Table 4 illustrates selected space operation characteristics. A high altitude, subparabolic orbit $(\mathrm{C} 3 \sim 0)$ space basing obviated the need for multi-week spiral escapes and captures at its origin and destination. This also lessened the operation limits that would otherwise be imposed on a vehicle that is a source of high-energy radiation and neutrons, particularly near populated areas such as a space station. These departure/arrival orbits could be low lunar, lunar-altitude, or Lagrange orbits at the Earth, and at high major moon or sub-parabolic orbit at the major planet destinations. It was envisioned that steady state operation (following initial assembly in LEO and pre-deployment of space infrastructure) would consist of direct flights to the outer planets, with only refueling operations taking place prior to return (Figure 6).

Space basing necessitated pre-deploying considerable infrastructure at each destination. (Designs for this infrastructure have not yet been examined, represent a significant issue for consideration for all such space based transportation systems, and could also prove to be development-lethal for fusion propulsion.) In-situ resource utilization (ISRU) plants on the waterice rich major moons of the outer solar system would generate the slush hydrogen propellant and the deuterium needed for fuel. Autonomous propellant tankage, the same systems used by the Discovery II, would also transport the propellant from the ISRU plants to the Discovery II. These same vehicles would initially transport propellant via HLLV's from Earth. The crew would use a dedicated, air breathing propulsion vehicle for transport from Earth's surface to a space station in LEO. From there, a small, high thrust inter-orbit shuttle would quickly transport the crew from LEO to the Discovery II.

Robotic atmospheric vehicles for mining ${ }^{3} \mathrm{He}$ would be needed should deposits on the surfaces of major moons prove insufficient. Few conceptual designs for such facilities exist, and their technical viability and cost effectiveness remain largely speculative. One study proposed a ${ }^{3} \mathrm{He}$ mining facility suspended on a balloon within Jupiter's atmosphere. ${ }^{14}$ This concept was capable of producing $\sim 1 \mathrm{gm} / \mathrm{sec}$ of ${ }^{3} \mathrm{He}$ and deuterium each by processing $680 \mathrm{~kg} / \mathrm{sec}$ (i.e. $3,600 \mathrm{~m}^{3} / \mathrm{sec}$ ) of atmosphere. This concept had a mass of $200 \mathrm{mt}$ and also required $10 \mathrm{MWe}$ of power. Ingenious in its design, it nevertheless illustrated the considerable difficulty in using in situ resources even though they may be plentiful.

Although the mission analysis assumed starting and stopping outside the effective gravity wells of the origin and destination planets, the sub-parabolic $\Delta \mathrm{V}$ propellant requirements were calculated for the Saturn mission. For an earlier version of the Discovery II to go out of and in to deep gravity wells, the additional propellant mass of $51 \mathrm{mt}$ for Earth escape and $22 \mathrm{mt}$ for Saturn orbit capture at Titan was calculated. The 
corresponding spiral out and spiral in trip times were estimated to be 10 days and 5 days respectively. Thus, Discovery II performing escape and capture maneuvers would have minor, though significant, effect on propellant loading and trip time.

\section{PAYLOAD}

\section{Overview}

In recent years, NASA's Human Exploration and Development of Space organization has begun to focus on two of the most difficult obstacles to long duration human interplanetary travel: the detrimental effects of weightlessness and radiation on the human body. These two areas will have significant impacts on human payload design studies, and are the main reason for the Discovery II's new payload system. In addition, the nominal crew size of six (with sufficient accommodations and supplies for twelve) necessitated a crew payload larger than most current concepts.
The crew modules were new designs, with subsystems scaled from a recent NASA Mars study. ${ }^{16}$ The design was compared to the International Space Station (ISS) Laboratory Module. This permitted a reasonable check of the mass properties for subsystems by way of habitable volume-to-mass scaling. The new module designs were found to be conservative by up to a factor of 3 when compared to ISS flight hardware. Other scalings were developed, such as volume-to-crew number and trip time-to-crew number-to-consumables, and used to develop estimates for the mass properties. Modifications were made, such as graphite-epoxy (GrEp) IM7/977-2 in lieu of aluminum to reduce mass due to GrEp's up to $\sim 55 \%$ lower density and $\sim 50 \%$ greater strength. Also, aluminum is well known as one of the least attractive structural materials for piloted interplanetary flight due to its greater propensity to generate secondary radiation products from solar and deep space radiation sources. All of the newly designed modules were sized on HLLV throw weight and corresponding payload fairing dimensions.

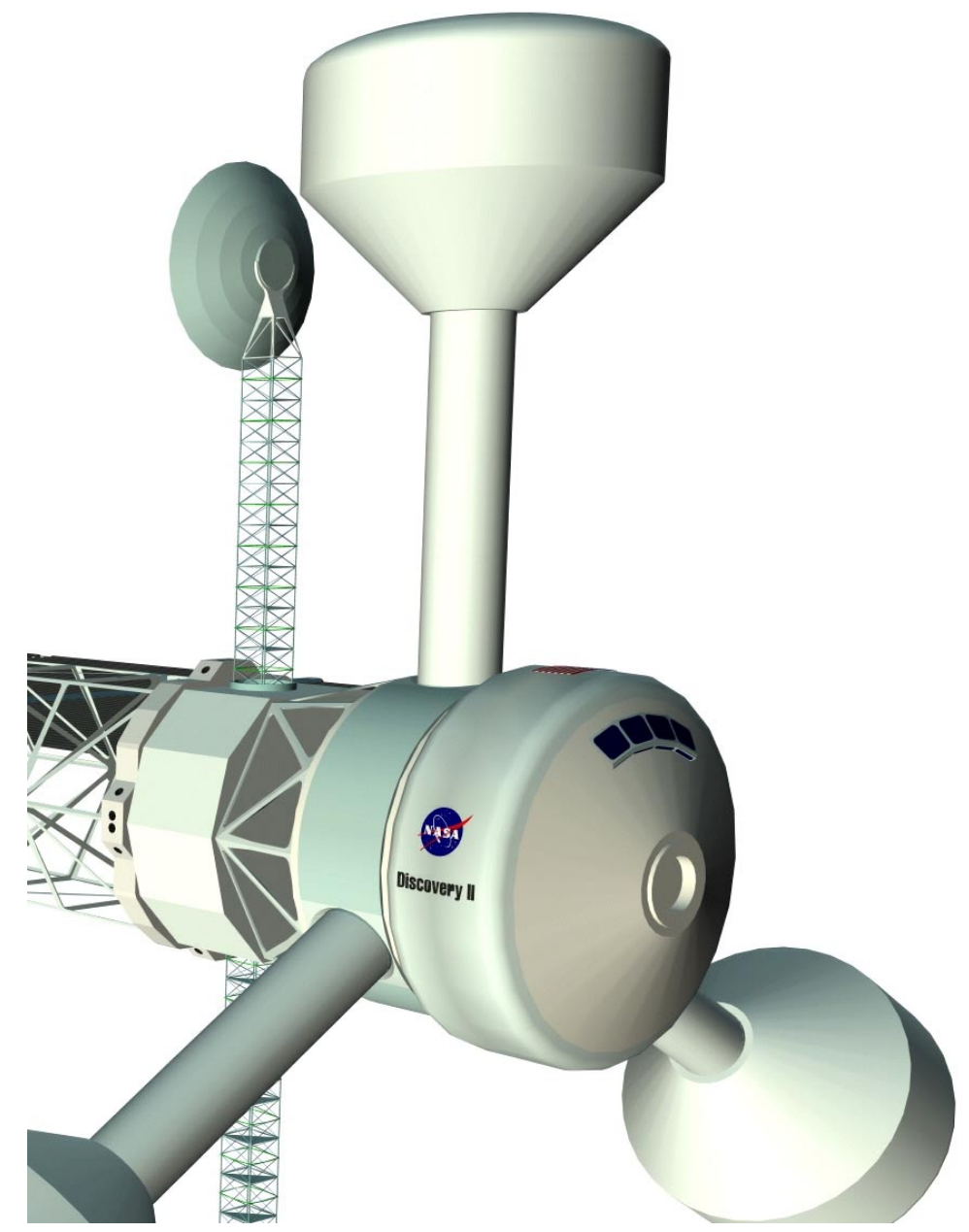

Figure 7: Artificial Gravity Crew Payload 
The crew payload was comprised of three rotating Laboratory/Habitation (Lab/Hab) Modules attached to the fixed Central Hub via three connecting Tunnels (Figure 7). A three-module configuration was recommended to reduce dynamic instability in the rotating structure. ${ }^{15}$ The Lab/Hab Modules were the primary laboratory and habitation facilities for the crew, and where most of the astronauts' time would be spent in a constant $0.2 \mathrm{~g}$ artificial gravity environment. They were $71 / 2 \mathrm{~m}$ in diameter, of sufficient height to permit a two-story layout, and each contained crew accommodations for at least four crewmembers, as well as scientific, health care, and recreation equipment. They also had independent life support, vehicle status, thermal, power, communication, and data systems. Consumables included sufficient air, water, and food for up to one year. ${ }^{16}$ For safety, each module was required to have two physically separate means of crew egress. For the Lab/Hab Modules, this meant the addition of an airlock outfitted with space suits.

Table 5: Payload Mass Properties

\begin{tabular}{|c|c|c|}
\hline \multirow[t]{12}{*}{ Structure } & & \\
\hline & Lab/Hab modules (3) & 7 \\
\hline & Central hub & 4 \\
\hline & Tunnels (3) & 2 \\
\hline & Airlocks (3) & 1 \\
\hline & Structural beef-up & 3 \\
\hline & Power management & 2 \\
\hline & Avionics & 1 \\
\hline & Life support & 12 \\
\hline & Thermal control & 4 \\
\hline & Rotation start/stop RCS & 2 \\
\hline & Payload adapter & $\sim 0$ \\
\hline \multirow[t]{6}{*}{ Shielding } & & \\
\hline & Storm shelter & 47 \\
\hline & Nominal radiation & 13 \\
\hline & Thermal & 1 \\
\hline & Micrometeoroid & 1 \\
\hline & Containment hull & 7 \\
\hline \multirow[t]{6}{*}{ Crew systems } & & \\
\hline & Accommodations & 8 \\
\hline & Consumables & 10 \\
\hline & Crew/suits & 3 \\
\hline & EVA equipment & 2 \\
\hline & Science & 2 \\
\hline Weight growth & Contingency & \\
\hline Total (mt) & & \\
\hline
\end{tabular}

The Central Hub served as the fixed interface for both the crew transport vehicle docking port (fore) and the fusion propulsion system (aft). These parts were inertially fixed, with the middle serving as the rotating link with the three Tunnels that provided access to the three Lab/Habs. The Central Hub was also heavily shielded to serve as the storm shelter for ambient high radiation events. The Tunnel internal diameters $(1.2 \mathrm{~m})$ permitted two crewmembers to pass in shirtsleeves (or single file in spacesuits).

The total habitable volume was $872 \mathrm{~m}^{3}$, of which the three Lab/Hab Modules provided $495 \mathrm{~m}^{3}$. Power required for nominal usage was $30 \mathrm{kWe}$. The total mass of the payload was $172 \mathrm{mt}$., which included a $30 \%$ weight growth contingency $(40 \mathrm{mt})$. (Table 5)

Defining and engineering the interface between the payload and the vehicle represents one of the most important, time consuming, and complex tasks in preparing for launching today's space missions. Customarily, the payload interface (adapter) mass is generally chargeable to the payload in terms of performance. However, in advanced concepts such as this, the concept of a payload adapter is somewhat ambiguous, and had a negligibly small relative impact on mass and power requirements anyway. As a result, the adapter's structure was envisioned as a simple truss assembly of negligible mass. Interface hardware (power connections, sensors, etc.) was also of negligible mass.

\section{Artificial Gravity}

There have been many thorough engineering studies of spacecraft for piloted interplanetary missions. The overwhelming majority have not included artificial gravity so as to simplify design and reduce development cost. There have been, however, some rotating concepts (where either a part or the entire vehicle rotated, contained an internal centrifuge, or used deployed tethers) that provided the centripetal acceleration to enable at least partial artificial gravity.

The detrimental effects of weightlessness on the human body have been observed for decades. Bone de-calcification and muscle atrophy represent leading threats to the health and effectiveness of astronauts on interplanetary missions, with other concerns still receiving medical speculation. It would be counterproductive if, after considerable effort to journey to their destination, the astronauts found themselves unable to perform their duties, even on a $<1 \mathrm{~g}$ body. Various exercise, diet, and medicinal countermeasures have been tried, but have usually proved unsatisfactory in mitigating the detrimental effects of weightlessness. ${ }^{17}$

The artificial gravity concept adopted for the Discovery II was a derivative of a previously created and published concept by the Martin Marietta Astronautics Corporation. ${ }^{18}$ In that design, two Space Station Habitation Modules were docked to a central 
hub by way of connecting tunnels. The entire vehicle (main propulsion, tankage, payload, etc.) was spun-up by a small RCS system to generate up to $1 \mathrm{~g}$ of artificial gravity. It had many advantages: varying rotational spin (thus gravity) to an appropriate amount throughout the journey; modular design facilitating ETO launch and assembly via in-space docking maneuvers; long rotation arm mitigating radial and tangential Coriolis force effects experienced by the crew; and avoidance of complexity and cost of a spun/de-spun central hub.

\section{Table 6: Payload System Characteristics}

\begin{tabular}{|c|c|}
\hline Nominal crew size & 6 to 12 \\
\hline Consumables (months) & 12 \\
\hline Number of habitable modules & 7 \\
\hline Module structural material & GrEp \\
\hline Lab/Hab, Central Hub diameter (m) & 7.5 \\
\hline Lab/Hab height (2 story) (m) & 5.6 \\
\hline Storm shelter height (m) & 7.8 \\
\hline Total habitable volume $\left(\mathrm{m}^{3}\right)$ & 872 \\
\hline Artificial gravity (g's) & 0.2 \\
\hline Rotation rate (rpm) & 3.25 \\
\hline Rotation arm (m) & 17 \\
\hline Maximum walking-to-rim speed ratio & 0.17 \\
\hline Radial gravity gradient (milli-g's/m) & 12 \\
\hline Nominal GCR maximum dose (rem/yr) & $\sim 55$ \\
\hline Storm shelter GCR maximum dose (rem/yr) & $\sim 30$ \\
\hline Radiation shielding (nominal) $\left(\mathrm{cm}\right.$ of $\left.\mathrm{H}_{2} \mathrm{O}\right)$ & 2 \\
\hline Radiation shielding(storm shelter) $\left(\mathrm{cm}\right.$ of $\left.\mathrm{H}_{2} \mathrm{O}\right)$ & 20 \\
\hline MLI thermal shielding (in) & 2 \\
\hline Micrometeoroid shielding (mm) & 0.5 \\
\hline Power consumption (steady state) (kWe) & 30 \\
\hline Mass (mt) & 172 \\
\hline
\end{tabular}

The amount of artificial gravity required for crew health has been the subject of considerable study. To date, however, insufficient experimental data exists to answer with certainty even the most basic design questions affecting human physiological wellbeing. It is this lack of basic human health effects data that drives spacecraft designers to consider other effects, such as locomotion, in order to at least bound the design problem. In general, it is thought that a minimum of $0.2 \mathrm{~g}$ ( lunar gravity) might be sufficient to at least facilitate locomotion based on the Apollo experience. Radial Coriolis forces can become a problem when the maximum walking speed is greater than $\sim 1 / 4$ of the maximum rim speed (if the motions are collinear). ${ }^{17}$ Tangential Coriolis forces can become a problem when an astronaut moves (or moves another object) in the radial direction (i.e., from the artificial gravity region towards the zero-g hub) when the gravity gradient changes significantly with respect to the height of the astronaut. Thus acceptable rotation speed and radius parameters are interrelated and require more assessment of human physiology and locomotion requirements. Human subjects have shown to be readily adaptable to rotation rates up to $6 \mathrm{rpm}$ (up to $10 \mathrm{rpm}$ if given specialized training). ${ }^{17}$ To minimize Coriolis effects, the corresponding minimum rotation arm was also thought to be at least $15 \mathrm{~m}^{17}$ It was thus decided that the Discovery II would retain the Martin Marietta design parameter of a $17 \mathrm{~m}$ rotation arm, though a lower rotation rate of $3.25 \mathrm{rpm}$ was used to produce an artificial gravity level at the Lab/Hab floor of only $0.2 \mathrm{~g}$. This combination satisfied the suggested guidelines (maximum walking-to-rim speed ratio of 0.17 and radial gravity gradient of $\sim 12$ milli-g's/m)(see Table 6 ), while satisfying reasonable ETO launch mass, volume, and other payload fairing constraints.

One-sixth-scale arcjet RCS thrusters, with two opposing thrusters mounted on each Lab/Hab Module, accomplished the spin-up (and any spin-down if ever required) following assembly. The fission reactor and primary RCS supplied the power and propellant for the RCS spin-up thrusters respectively. It was anticipated that once set in motion, the rotating payload would remain rotating indefinitely (except for repairs). Further definition of the spin-up sequence was deferred.

\section{Shielding from Ambient Radiation}

Multi-month trip times through interplanetary space have the concomitant danger of exposure to lethal doses of ambient radiation unless the crew payload is adequately shielded. The most serious concerns lie with galactic cosmic rays (GCR) and solar proton flares (both ordinary and large). GCR's are stripped atomic nuclei (primarily $\mathrm{Z}=1,2$ at $\mathrm{GeV}$ energies) that originate in interstellar space and penetrate our sun's magnetosphere. ${ }^{19}$ They can vary in strength by several orders of magnitude, are isotropic, and are somewhat periodic in time due to modulation by the sun's cycle. Flares are high-energy protons ejected from the sun throughout its 11 year solar cycle (55 occurred during the solar cycle XXI (1975-86)). " "Ordinary flares" have been classified as having at least an integral fluence of $10^{7} / \mathrm{cm}^{2}$ with energies above $10 \mathrm{MeV}$. Large solar proton flares (referred to as "anomalously large events"), however, can occur during the peak years of the solar cycle and produce more protons than all ordinary flares in the cycle combined. ${ }^{19}$ The seriousness of radiation exposure is a function of probability of occurrence, energies of primary radiation, exposure time, shielding material, interaction with body parts, personal factors, and others factors. 
The National Council on Radiation Protection and Measurements (NCRP) has recommended that an astronaut not receive a depth-dose equivalent greater than $50 \mathrm{rem} / \mathrm{yr} .^{20}$ Further, total lifetime limits have been set to between 100 and 400 rem, depending on several personal factors. But NCRP has recommended that these standards apply to LEO only, and would review any piloted interplanetary mission in the future in order to establish appropriate standards. Thus, LEO values were used as design guidelines only.

The ambient radiation shielding design for the Discovery II was adopted from a recent NASA Mars mission design study. ${ }^{19}$ That 500-day mission study included two anomalously large (solar proton flare) events occurring at less than 1 AU (i.e. during a Venus swing-by). It therefore represented a harsh environment, certainly when compared to trajectories largely confined to the outer solar system. The resultant radiation doses for that mission and shield design were found to be $<70$ rem. However it should be noted that the analysis ground rules included $1 / 3$ of the crew's time (presumably during sleep periods) to be spent in a heavily shielded storm shelter.

Because the Discovery II operated outside of Earth's protective magnetosphere, it was assumed considerable shielding would be required. The nominal radiation shield for the Discovery II consisted of a layer of liquid water surrounding all modules, enveloped by a GrEp containment hull wrapped by 2 inch multi-layer insulation (MLI) thermal protection and $0.5 \mathrm{~mm}$ aluminum micrometeoroid shielding. Water was chosen due to the low atomic mass of hydrogen and the resultant low generation of secondary radiation products. $2 \mathrm{~g} \mathrm{H}_{2} \mathrm{O} / \mathrm{cm}^{2}$ was used for nominal shielding on all habitable parts of the payload except the storm shelter. This provided $\sim 55 \mathrm{rem} / \mathrm{yr} 5-\mathrm{cm}$ depth dose equivalent for GCR (at solar minimum), which slightly exceeded the LEO guideline (see Table 6). ${ }^{20}$ The storm shelter had robust shielding $\left(20 \mathrm{~g} \mathrm{H}_{2} \mathrm{O} / \mathrm{cm}^{2}\right)$, which provided $\sim 30 \mathrm{rem} / \mathrm{yr} 5-\mathrm{cm}$ depth dose equivalent for GCR (at solar minimum), but was achieved at a considerable mass penalty of $>47 \mathrm{mt}$ (see Table 5).

Others have proposed potential ancillary benefits of this design. ${ }^{21}$ Even without a separate micrometeoroid shield, a water layer could provide selfsealing protection from meteoroids by freezing and plugging punctures. Also, low quality waste heat from electronics, human use, or possibly low temperature refrigeration systems might be effectively dumped into a water shield rather than rejected to space through inefficient, low temperature radiators.

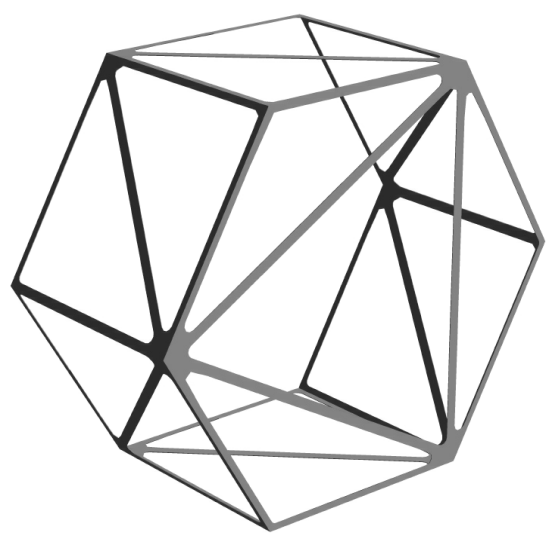

Figure 8: Central Truss Section

\section{CENTRAL TRUSS}

The primary structure linking the major systems of the vehicle was chosen to be a truss network in order to minimize mass while retaining strength. The lightweight truss material was Aluminum Graphite/Epoxy (Al GrEp). This material and the strut/joint/node design were adopted from a tested, earlier concept for the International Space Station. The truss cross section was changed from a square to a hexagon in order to accommodate various numbers and arrangements of propellant tanks. A structural strut from a prior design was used, where its length had been reduced from $5 \mathrm{~m}$ to $3.5 \mathrm{~m}$, yet the overall cross-bracing arrangement (one diagonal per section) was retained. Figure 8 illustrates one truss section. Table 7 contains various characteristics of the truss network ${ }^{22}$. Despite a central truss length of $203 \mathrm{~m}$ (58 sections), the total mass was only $4.4 \mathrm{mt}$. Axial and lateral loading, however, necessitated a modest redesign.

The axial loading was assumed to be greatest at the aft end, where the propulsive thrust would have to be applied to the vehicle. A separate thrust structure that was designed to take an earlier designed 5,567 $\mathrm{lb}_{\mathrm{f}}$ thrust load and distribute it axially into the six longitudinal struts was still considered adequate. Its mass was minimal, with the 18 truss struts of radii 1.36 times greater than the baseline.

Table 7: Central Truss Characteristics

\begin{tabular}{lc}
\hline Material & Al GrEp \\
Total length (m) & 203 \\
Length per section (m) & 3.5 \\
Number of sections & 58 \\
Number of struts, nodes, joints per section & 97 \\
Mass (mt) & 6 \\
\hline
\end{tabular}


Given the low bending moment limit of the struts (scaled from the original $5 \mathrm{~m}$ strut cantilever failure at $\left.1,024 \mathrm{ft} \mathrm{lb}_{\mathrm{f}}\right)^{22}$, the lateral loads were of more concern. An earlier structural loading design was retained, since its assumptions were conservative with respect to the current, smaller radiator panel. In that assessment, each radiator had a mass of $\sim 26 \mathrm{mt}$, at an average total moment arm of $50 \mathrm{~m}$, had to be attached at each node and at each strut midpoint to reduce the bending moment to a level comparable to the scaled limit value. This case was of greatest concern at the maximum acceleration, 1.9 milli-g's, encountered at end of mission. The other systems produced lower moments due to lower masses and/or small moment arms. Analysis of torsion loads into the central truss due to unbalanced torques generated by start-up of the power system was deferred. A mass estimate for a flywheel momentum storage system was included, however, to arrest those loads and is discussed later. The mass of the central truss network was $6 \mathrm{mt}$, which included ample allowance for attachment hardware (set equal to onehalf that of the entire central truss.)

\section{FUSION REACTOR}

\section{Overview}

Unlike today's terrestrial reactors that must be safely isolated from the biosphere, a largely skeletal design was used for the Discovery II. Smaller in size than current research reactors, the design philosophy was to minimize mass, maximize useful charged power out, and facilitate direct radiation of waste power to space without a containment vessel. An ignited plasma operating mode was chosen, as well as high bootstrap current, in order to minimize the re-circulating power fraction required and the concomitant conversion system mass for generating injection power. It was thought that a continuously thrusting propulsion system would be better served by this mode of reactor operation, where charged transport power was used exclusively for propulsion purposes. A small major radius $(2.48 \mathrm{~m})$ and small aspect ratio (2.0) reactor geometry was chosen to minimize size and mass. The scaling for toroidal beta $\left(\beta_{\mathrm{T}}\right)$ favored elongated $(3: 1)$, compact devices with large plasma currents $(\sim 66 \mathrm{MA})$

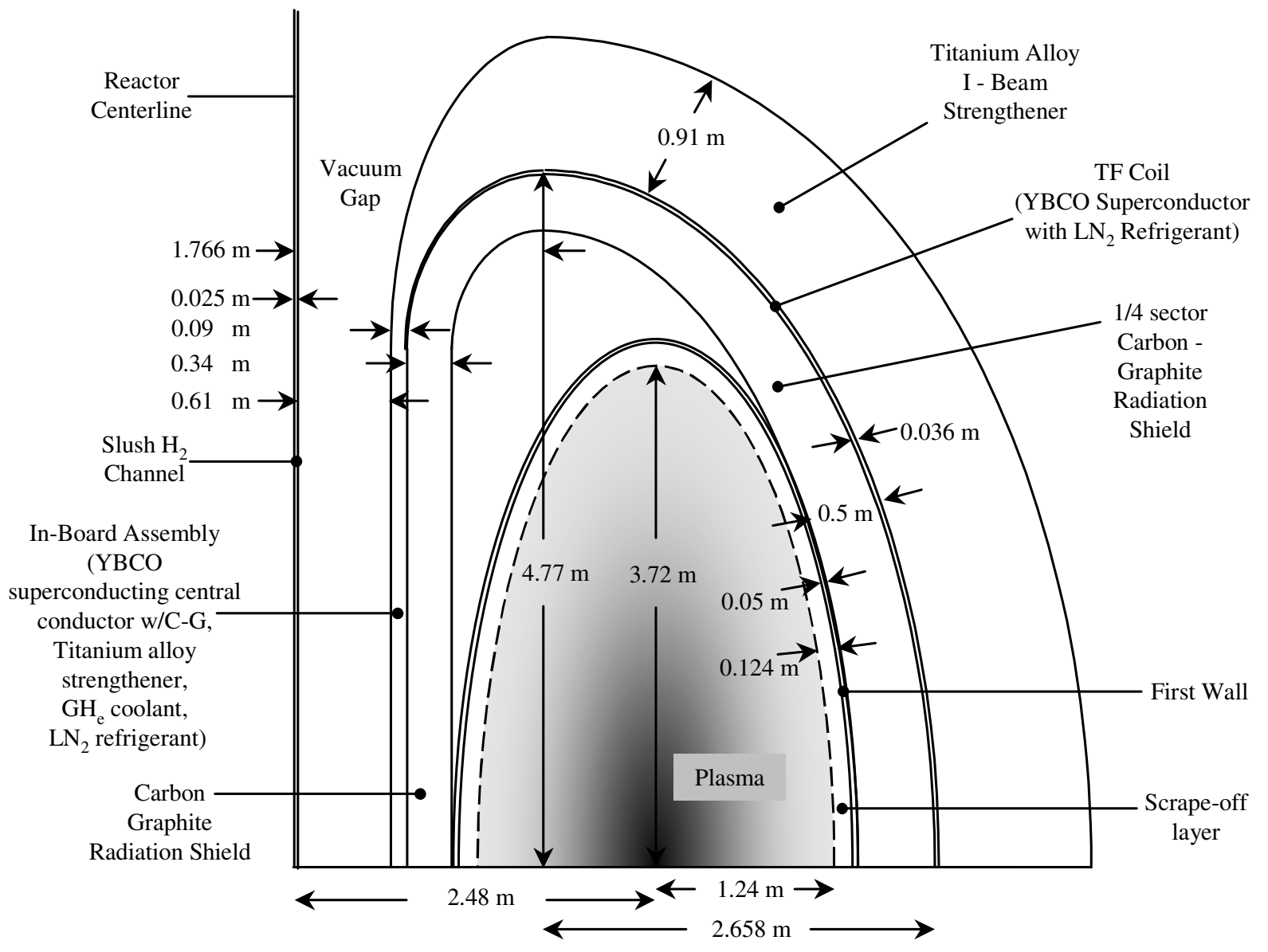

Figure 9: Fusion Reactor Radial Build 
and moderately large centerline magnetic fields (8.9 T). These magnetic field requirements led to very large toroidal field (TF) coil currents (9.2 MA). Twelve TF coils were used to generate the toroidal magnetic field; seven poloidal field (PF) coils were used to provide plasma stability. Existing materials were used with occasional, extrapolations of physical properties, evaluated at operating temperatures.

Figure 9 illustrates the radial build, including an upper half of one TF "D-shape" coil and its inboard assembly. Beginning with the reactor centerline and working outboard, an annulus along the centerline provided a flow channel for the slush hydrogen propellant. A vacuum gap separated it from a Titanium alloy compression structure that provided support for the current-induced coil loads. A high temperature superconductor coating on a composite structure supported the large current that generated the toroidal magnetic field. Several coolant and refrigeration channels provided heat transfer for absorbed radiation and maintained cryogenic temperatures. A carbon composite $/ \mathrm{W}_{2} \mathrm{~B}_{5}$ shield attenuated most of the radiation that was transmitted through the first wall. A double hull, carbon composite first wall was coated with optical grade beryllium on the plasma-facing surface. A scrape off layer of ash was assumed to be $10 \%$ of the $1.24 \mathrm{~m}$ minor radius. The outboard structures were similar. A slightly thicker, quarter sector radiation shield was used to shadow the TF coil from the core. Behind it was a channel for heat transfer. The TF coil was jacketed with refrigerant to maintain cryogenic conditions. A large titanium alloy I-beam strengthener was used to counter the tension loads generated by the TF coil currents. Further outboard (not shown) were the PF coils and supporting equipment (including support for the high harmonic fast wave heating system).

\section{Plasma Modeling and Characteristics}

Modeling of the plasma conditions was performed through a 1-D plasma power balance computer program ${ }^{23}$. It was designed to analyze generic, small-to-large aspect ratio, tokamak fusion reactors, inductive and non-inductive heating, driven and ignited operation, burning DT, DD, or $\mathrm{D}^{3} \mathrm{He}$ fuels. By pursuing peaked temperature and number density profiles within the core of a plasma, a relatively small fusion-producing region was established, satisfying Lawson and ignition criteria without necessitating large beta $(\beta)$ throughout the plasma. The lower temperature and number density outer regions would contribute to a volume-averaged $\beta$ value within MHD stability limits. This approach was tremendously attractive for space propulsion applications where compact size, thus reduced mass, is of paramount importance. Profile shape factors $(\delta)$ for temperature (T), number density (n), and current density (J) were of the functional form given in Equation (4), and were integrated along the minor radius ( $\mathrm{r}$ ) (where 0 $\leq \mathrm{r} \leq \mathrm{a})$ where a concentric ellipse approximation was used.

$$
\begin{gathered}
x(r)=<x>f(r) \quad \text { where } \\
x=(T, n, J) \\
f(r)=\left(1+\delta_{T, n, J}\right)\left(1-r^{2} / a^{2}\right)^{\delta_{T, n, J}} \\
x=\frac{2}{a^{2}} \int_{0}^{a} x(r) r d r
\end{gathered}
$$

Charged particle and neutron power density, including DD side reactions, were integrated as functions of number density and radius (along with temperature dependent reactivities). Bremsstrahlung and synchrotron radiation power densities were also integrated as functions of temperature and number density (and thus radius). From these quantities, other primary reactor characteristics such as plasma current, magnetic field, confinement time scaling, etc., were solved for while satisfying constraints such as critical beta and plasma power balance. Volume averaged quantities such transport power and radiation loss were used to determine initial available charged power for propulsion and available waste power for conversion to auxiliary electrical power respectively. Plasma transport loss power, which included convection and conduction loss, represented the primary source of fusion reaction charged products for propulsion application and thus the quantity to be maximized.

$\mathrm{D}^{3} \mathrm{He}(1: 1$ ratio) was chosen as the reactor fuel in order to maximize the charged transport power output and minimize neutron output power fraction. It was decided that in the time frame of this concept, reactor operation at a plasma temperature of $50 \mathrm{keV}$ would represent only an incremental technological challenge over that of a DT-based concept operating at $10 \mathrm{keV}$ (and a fuel significantly more conducive to space propulsion application). Also, as was discussed earlier, solar system-class operation presupposed propellant and fuel supply availability in the hydrogen and helium-rich outer planet atmospheres and satellites, mitigating supply issues surrounding ${ }^{3} \mathrm{He}$.

$\mathrm{D}^{3} \mathrm{He}$ fuel with a spin vector polarized parallel to the magnetic field was used to capitalize on the up-to $50 \%$ enhancement in fusion reactivity cross section, ${ }^{24,25}$ 
tremendously improving the charged output power. The methods of creating and utilizing polarized fuel have been developed through theory (DT and $\mathrm{D}^{3} \mathrm{He}$ ) and experiment $(\mathrm{DT})^{24,25}$. Although much work remains on maintaining fuel polarization, it is well known that ionization and atomic collision processes cannot result in depolarization ${ }^{25}$. However, there are potentially significant design impacts to the first wall and fuel injection that will be discussed later.

Table 8: Fusion Reactor Characteristics

\begin{tabular}{lc}
\hline Major radius $(\mathrm{m})$ & 2.48 \\
Minor radius $(\mathrm{m})$ & 1.24 \\
Aspect ratio & 2.0 \\
Elongation & 3.0 \\
Plasma volume $\left(\mathrm{m}^{3}\right)$ & 225.8 \\
Safety factor $(\mathrm{edge})$ & 2.50 \\
Safety factor $(\mathrm{axis})$ & 2.08 \\
Fuel ion density $\left(10^{20} / \mathrm{m}^{3}\right)$ & 5.0 \\
Electron density $\left(10^{20} / \mathrm{m}^{3}\right)$ & 7.5 \\
Plasma temperature $(\mathrm{keV})$ & 50 \\
Volume averaged beta & 0.318 \\
Confinement time $(\mathrm{sec})$ & 0.552 \\
Average neutron wall load $\left(\mathrm{MW} / \mathrm{m}^{2}\right)$ & 1.03 \\
Average radiation wall load $\left(\mathrm{MW} / \mathrm{m}^{2}\right)$ & 5.20 \\
Ignition margin & 1.235 \\
Toroidal magnetic field (centerline) $(\mathrm{T})$ & 8.9 \\
Maximum magnetic field (coil surface) $(\mathrm{T})$ & 32.3 \\
Gain factor $(\mathrm{Q})$ & 73.1 \\
Plasma current $(\mathrm{MA})$ & 66.22 \\
Bootstrap current fraction (overdriven) & 1.16 \\
Wall reflectivity (effective) & 0.98 \\
Number density profile shape factor & 1.0 \\
Temperature profile shape factor & 2.0 \\
\hline
\end{tabular}

Table 8 illustrates selected reactor and plasma characteristics. No energy confinement time $(\tau)$ scaling law exists based on experimental data for an ignited, steady state thermonuclear device. However, an attempt was made to calibrate the reactor's operating characteristics with known total energy confinement time relations. A $\tau=0.552 \mathrm{sec}$ was in good agreement with the 1992 International Thermonuclear Experimental Reactor (ITER) H-mode scaling law $(0.565 \mathrm{sec})$ that is a function of plasma current $\left(\mathrm{I}_{\mathrm{p}}\right)$, toroidal magnetic field $\left(\mathrm{B}_{\mathrm{T}}\right)$, number density $(\mathrm{n})$, total plasma heating power $\left(\mathrm{P}_{\mathrm{L}}\right)$, average atomic mass $(\mathrm{A})$, major radius $(\mathrm{R})$, minor radius $(\mathrm{a})$, and elongation $(\kappa)$ (Equation (5)) ${ }^{26}$.

$$
\tau=0.021 I_{p}^{0.55} B_{T}^{0.91} n^{0.17} P_{L}^{-0.55} A^{0.5}(R / a)^{-0.19} R^{2.3} \kappa^{0.7}
$$

A somewhat shorter confinement time was obtained from the 1997 ITER L-mode scaling $\operatorname{law}^{27}$ of $\tau=$ $0.216 \mathrm{sec}$. The primary driving terms in the $\tau$ scaling were found to be the large values of plasma current, applied magnetic field, total plasma heating power, and major radius. Although it is not clear which sub-ignited, experimental database-derived scaling law would be more representative of ignited plasma conditions for a propulsion system, the proposed concept appeared consistent with current relationships.

A density weighted, volume averaged plasma $\mathrm{T}$ of $50 \mathrm{keV}$ and $\mathrm{a} \mathrm{n}_{\mathrm{i}}$ of $5 \cdot 10^{20} \mathrm{~m}^{-3}$ were chosen. Representative $\mathrm{n}$ and $\mathrm{T}$ profile shape factors of 1.0 and 2.0 respectively were chosen to enhance fusion power production. A sufficiently high critical beta $\left(\beta_{\text {crit }}\right)$ $(\sim 30 \%)$ was chosen to efficiently use the strong magnetic field and reduce synchrotron losses. An edge safety factor of 2.5 was chosen.

$$
\begin{gathered}
I_{p}=\frac{\beta_{c r i t} a B_{T}}{\left(\frac{\beta_{N}}{100}\right)} \\
P_{\text {inject }}=\frac{I_{p}\left|f_{b s}-1\right|}{\eta_{R F}}
\end{gathered}
$$

The $\beta_{\text {crit }}$ constraint was satisfied by requiring the total plasma current to be given by Equation (6) (with the Troyon coefficient $\left(\beta_{\mathrm{N}}\right)$ of 5 , based on a recent analytic study $^{28}$ ) for a low aspect ratio tokamak.) The large $\beta_{\text {crit }}$ and magnetic field required for space propulsion application suggested that great leverage existed with maximizing $\beta_{\mathrm{N}}$ in order to minimize large $\mathrm{I}_{\mathrm{p}}$ and its impact on reactor design.

An injected power $\left(\mathrm{P}_{\text {inject }}\right)$ of $108 \mathrm{MW}$ on startup was used in Equation 7. This was consistent with a Bootstrap (plasma) current overdrive fraction $\left(f_{b s}\right)$ of 1.16 and an external current drive efficiency $\left(\eta_{R F}\right)$ of 0.1 A (driven)/W (injected). Though self-consistent, the interrelationship between bootstrap current overdrive, fueling rate, and Coaxial Helicity Ejection is yet to be quantitatively understood and is the subject of a related, future experiment campaign.

\section{Bootstrap Current Overdrive}

The significantly greater fusion product $\mathrm{nT} \tau$ for a $\mathrm{D}^{3} \mathrm{He}$ fueled reactor led to the requirement for large plasma current $(\sim 66 \mathrm{MA})$ in order to obtain sufficient confinement. To provide for this current, a 
diffusion-driven bootstrap current, first experimentally observed in large tokamaks, was relied upon. The large $T_{i}$ and $n_{i}$ present in the core of $D^{3}$ He fueled reactors could benefit from significant, arbitrarily large $(90 \%+)^{29}$, bootstrap fractions where only modest seed currents provided by external heating would be needed. Bootstrap current overdrive (where the selfdriven plasma current exceeds the required current) appeared to be an attractive means to facilitate operational control of the plasma current. By adjusting fueling rates, bootstrap current overdrive can assist in adjusting exhausted kinetic power by controlling plasma current. $^{30}$ Without "2-D equilibrium calculations to show how bootstrap current overdrive works, [and] since the profile effects dominate," 30 a simple, average bootstrap current fraction was recommended to be a value between 1.1 and $1.2,{ }^{30}$ slightly greater than predicted assuming an already demonstrated $\beta_{\mathrm{N}} \geq 6$. $^{31}$

Because the overdriven plasma current's radial profile usually will not align with the desired equilibrium profile, supplementary heating is required to both augment and negate regions of the plasma current. It was assumed that this heating could be minimized or even eliminated through the careful adjustment of bootstrap current overdrive, fueling rate, and Coaxial Helicity Ejection (described later) operating points. As a result, no external, steady state plasma heating was assumed to be necessary. This was a tremendous advantage to a propulsion system, since the otherwise required $\sim 100$ MWe steady state injection power would necessitate large, heavy, power conversion and waste heat rejection systems.

\section{Power Generation and Utilization}

Figure 10 illustrates the fusion power output and utilization. (The auxiliary fission reactor and its power flow are described separately.) Of the 7,895 MW of fusion power produced, $96 \%$ was in the form of charged particles with the remainder in (largely $2.45 \mathrm{MeV}$ ) neutrons. More than $3 / 4$ of the power out of the reactor $(6,037 \mathrm{MW})$ was charged transport power, (D and He ions, protons, and electrons) used solely for direct propulsion via the magnetic nozzle system. Synchrotron power (535 MW) was either absorbed by the first wall or reflected out the divertor channel to space. Much of the Bremsstrahlung (1,016 MW) and neutron radiation (307 MW) was absorbed throughout the reactor. Most of the heat generated by absorbed radiation $(1,119 \mathrm{MW})$ was transferred through a fancirculated, gaseous helium $(\mathrm{GHe})$ coolant system to a high temperature radiator. The remaining heat from absorbed radiation (96 MW) was converted to electrical power for onboard use ( 29 MW). No steady state

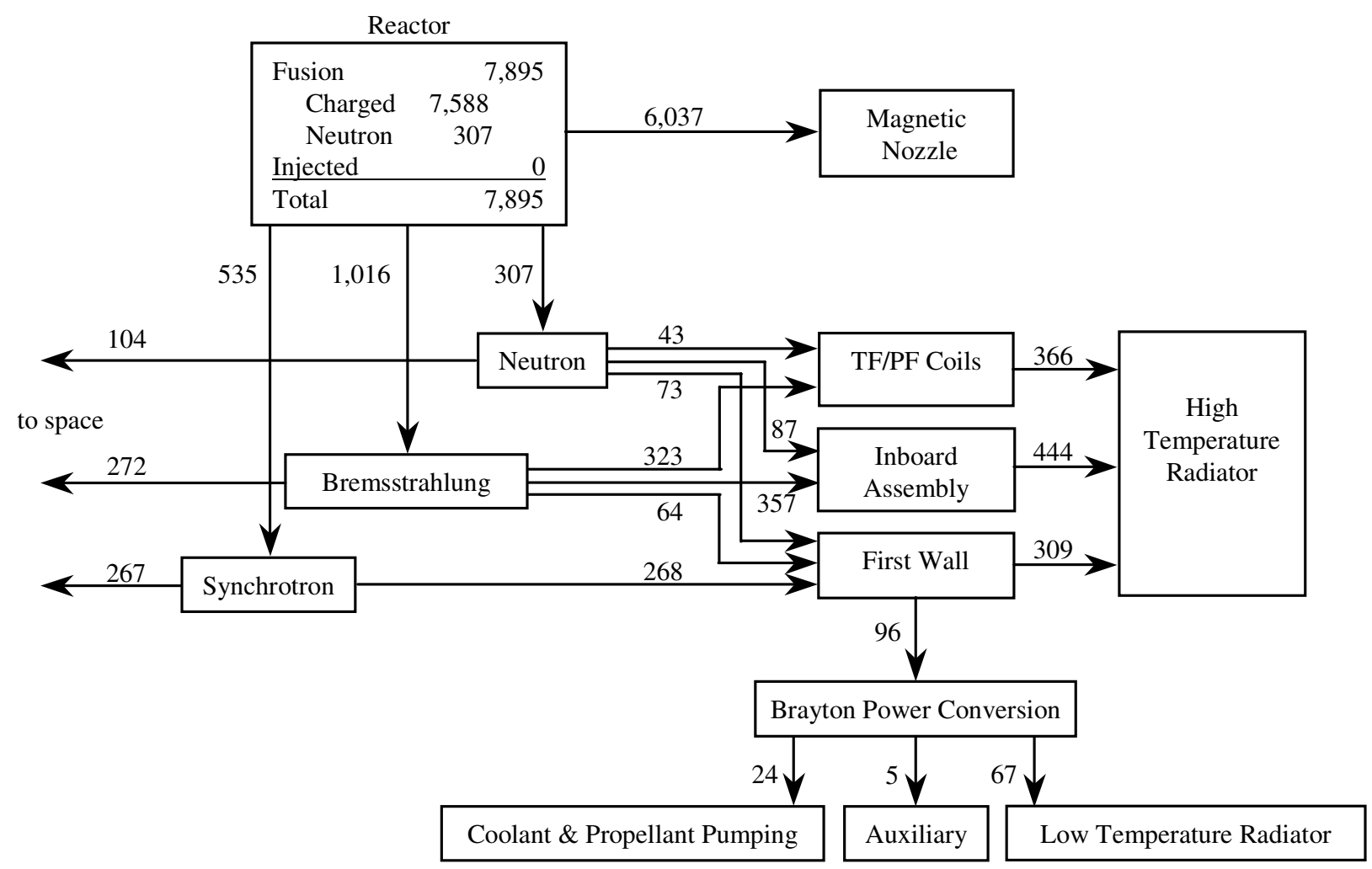

Figure 10: Fusion Power (MW) Output and Utilization 
injection power was necessary due to the ignited, overdriven bootstrap current operation of the reactor system. The Q-value (fusion power/input power) based on the $108 \mathrm{MW}$ of High Harmonic Fast Wave (HHFW) start-up heating was $>73$. Bremsstrahlung/synchrotron and neutron radiation wall loadings were 5.20 and $1.03 \mathrm{MW} / \mathrm{m}^{2}$ respectively.

Table 9: Auxiliary Power Usage (Nominal (fusion) and Startup (fission))

\begin{tabular}{|c|c|c|}
\hline & Nom & Startup \\
\hline Electron cyclotron resonance heating & 0 . & 1. \\
\hline $\mathrm{TF} / \mathrm{PF} /$ diver/mag noz coil refrigeration & 3.95 & 0.39 \\
\hline Fuel injector & 0.507 & 0.102 \\
\hline Battery recharge & 0.16 & 0.125 \\
\hline Communications & 0.2 & same \\
\hline Propellant/fuel tankage refrigeration & 0.133 & same \\
\hline Payload & 0.03 & same \\
\hline Avionics & 0.02 & same \\
\hline Total (MWe) & 5. & 2. \\
\hline
\end{tabular}

The choice of fuel and design of some of the reactor components was intended to maximize the fraction of useful, charged power-out while minimizing the fraction of power lost to (and the mass devoted to managing) radiation. This permitted the dedication of all charged power to propulsion while scavenging only absorbed waste radiation to produce electrical power through a heat cycle. Since the electrical power that could otherwise be produced at high efficiency was in excess of requirements, the reactor was deliberately designed to permit as much radiation as possible to escape directly to space $(643 \mathrm{MW})$. Electrical power requirements were largely for the motor/fan-circulated GHe coolant system for the reactor (24 MW). The remaining power was consumed by propellant pumping and other auxiliary usage (superconducting coil and propellant tankage refrigeration, startup/re-start battery recharge, fuel injector, avionics, communications, and payload). Table 9 summarizes the nominal auxiliary power usage provided by the fusion reactor $\left(5 \mathrm{MW}_{\mathrm{e}}\right)$ and startup/emergency re-start power usage provided by the fission reactor $\left(2 \mathrm{MW}_{\mathrm{e}}\right)$.

\section{First Wall}

The design philosophy for the first wall was two fold. First: minimize the absorption of Bremsstrahlung and neutron power to permit direct radiation to space for the outward-bound radiation. Second: provide a highly polished surface to enable multiple reflections of synchrotron radiation thereby facilitating re-absorption within the plasma. This approach reduced the magnitude of mass-intensive heat rejection equipment as well as synchrotron losses associated with burning advanced fuels at high $\mathrm{T}_{\mathrm{e}}$. Critical to the success of this approach was the choice of beryllium coating on carbon-graphite composite.

A metal conductor of low atomic number and capable of being polished to a high degree of reflectivity was essential. Optical grade beryllium was chosen for the plasma-facing surface due to its low mass absorption coefficient to $\mathrm{x}$-ray/gamma radiation, greater than $99 \%$ reflectivity at synchrotron radiation wavelengths ${ }^{32}$, existing manufacturing experience, and application within fusion research. The highest yield strength optical grade was selected: Brush Wellman $\mathrm{I}-250(97.5 \% \mathrm{Be}, 2.5 \% \mathrm{BeO}){ }^{33,34}$

The amount of synchrotron radiation absorbed by the first wall was calculated through an algorithm that related the physical properties of the wall material to those of the plasma. ${ }^{35}$ The angular, polarization, and spectrum frequency of the incident radiation was coupled to the response of the metallic reflector. Under plasma conditions satisfied by the Discovery II, the ratio of absorbed-to-generated synchrotron radiation $\left(\mathrm{K}_{\mathrm{c}}\right)$ was shown to be a function of $T_{e}, n_{e}, B_{T}$, the perpendicular distance across the reflector (1), the electrical resistivity $(\rho)$, and the geometry of the wall. $\mathrm{K}_{\mathrm{c}}$ was calculated using Equations 8, 9, and 10.

$$
\begin{gathered}
K_{c}=e^{a_{0}} T_{e}^{\left(a_{1}+a_{2} \ln \left(T_{e}\right)\right)} \\
a_{i}=\sum_{n=0}^{4} C_{n} \eta^{n} \\
\eta=\log _{10}\left(\frac{n_{e} l}{B_{T} \sqrt{B_{T} \rho}}\right)-19
\end{gathered}
$$

The wall reflectivity was calculated to be in excess of $99.5 \%$, which was consistent with published data for beryllium I-250. ${ }^{32}$ Thus, the first wall absorbed $268 \mathrm{MW}$ of synchrotron power. In addition, the total projected plasma-facing frontal area of the divertor channel and other systems was estimated to be $\sim 5 \%$. Assuming all synchrotron radiation incident on the divertor passed through the largely skeletal structure and out the magnetic nozzle with little probability of back reflection, an approximately equal amount of synchrotron power was lost through this process. Thus the total synchrotron power lost was $535 \mathrm{MW}$.

The large quantity of power absorbed by the plasma facing material necessitated heat transfer by a gaseous helium (GHe) coolant for transport to a radiator. GHe was chosen due to its inertness (thus benign to turbomachinery) and very high temperature capability. Its low heat capacity due to its low density necessitated high-pressure operation (7.5 MPa). 


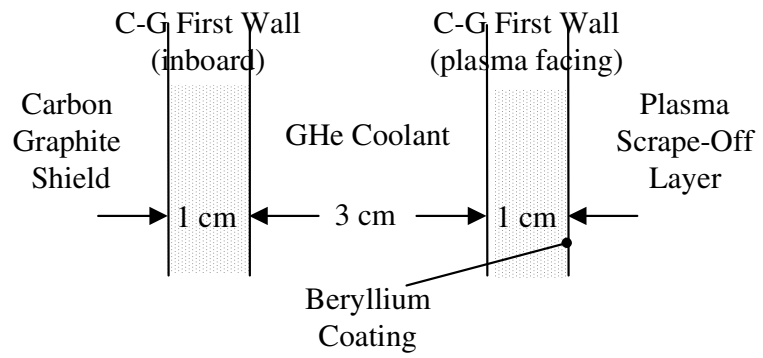

Figure 11: First Wall

Table 10: First Wall Characteristics

\begin{tabular}{lc}
\hline Configuration & $\begin{array}{c}\text { Double hull } \\
\text { Carbon-Graphite } \\
\text { Structural material }\end{array}$ \\
Plasma facing material & $>99.5$ \\
Reflectivity $(\%)$ & 1,824 \\
Structure temperature $\left({ }^{\circ} \mathrm{K}\right)$ & 405 \\
Power absorbed $(\mathrm{MW})$ & 5.20 \\
Wall loading (radiation) $\left(\mathrm{MW} / \mathrm{m}^{2}\right)$ & 1.03 \\
Wall loading (neutron) $\left(\mathrm{MW} / \mathrm{m}^{2}\right)$ & 0.034 \\
Maximum thermal stress $(\mathrm{GPa})$ & 1.060 \\
Maximum pressure stress $(\mathrm{GPa})$ & 5 \\
Reflecting surface area loss $(\%)$ & 14 \\
Mass (mt) & \\
\hline
\end{tabular}

To accommodate high pressure, a high strength material at high temperature and low density made carbon-graphite (C-G) composite an attractive first wall structural material, facilitating efficient heat transfer. C-G also served to minimize Bremstrahlung and neutron absorption, by enabling a thin wall design. The composite material would require a suitable coating due to its porosity, but this issue was deferred to future study. Today's multidirectional and unidirectionally reinforced carbon-graphite matrix composites have high tensile strength ( $\sim 0.4$ to $1.1 \mathrm{GPa}$ respectively) at high temperature $\left(\sim 1,900\right.$ to $2,300{ }^{\circ} \mathrm{K}$ respectively). ${ }^{36}$ Given the rapid improvement and usage of composites, it was assumed that in the time frame of the Discovery II, multidirectionally reinforced C-G matrix composites would be available with tensile strengths comparable to today's unidirectionally-reinforced C-G matrix composites (i.e. a factor of 3 improvement). Thus a multidirectionally reinforced $\mathrm{C}-\mathrm{G}$ matrix composite material was used to fabricate a "double hull", elongated toroidal shell first wall, since this material could be designed to accommodate high loads in any orientation. The inner and outer hulls were $1 \mathrm{~cm}$ thick separated by a $3 \mathrm{~cm}$ GHe coolant channel. Figure 11 illustrates the first wall design. Thermal and hoop stresses, radiation power absorbed, and heat transfer were evaluated. The resulting design characteristics and others are summarized in Table 10.
The thickness of the first wall structure satisfied thermal stress $\left(\sigma_{\text {thermal }}\right)$ limits and pressure limits $\left(\sigma_{\text {hoop }}\right)$ (approximated by thin-wall criteria). These limits were evaluated through Equations 11 and 12 (where coefficient of thermal expansion $(\alpha)$, Young's Modulus (E), thermal conductivity (k), Poisson ratio $(v)$, surface and volumetric heating rates $\left(\mathrm{W}_{\mathrm{s}}, \mathrm{W}_{\mathrm{n}}\right)$, wall thickness $(\mathrm{x})$, coolant pressure $(\mathrm{P})$, and radius $(\mathrm{r}))^{51}$ The maximum thermal stress within the first wall vessel was calculated to be $0.034 \mathrm{GPa}$, where pure volumetric heating was assumed $\left(\mathrm{W}_{\mathrm{s}}=0\right)$ and calculated to be over $50 \mathrm{MW} / \mathrm{m}^{3}$. This thermal stress was well below the limit even for today's multi-directional C-G composites. The maximum pressure stresses for the first wall and inboard assembly coolant channel inner wall were calculated to be 1,060 and 1,043 $\mathrm{MPa}$ respectively, which were less than tensile limits for current unidirectional C-G composite materials. The total mass of the double hull first wall was calculated to be $14 \mathrm{mt}$ (Table 10).

$$
\begin{gathered}
\sigma_{\text {thermal }}=\left(\frac{\alpha E}{2 k(1-v)}\right)\left(W_{s}+\frac{W_{n} x}{2}\right) x \\
\sigma_{\text {hoop }}=\frac{P r}{x}
\end{gathered}
$$

Several concerns regarding the use of beryllium within a fusion reactor environment were considered. Although I-250 had one of the highest $\mathrm{BeO}$ contents of all grades, the otherwise lack of an oxygen environment (with steady state as opposed to cycling operation) was thought to minimize potential cracking. ${ }^{37,38}$ Thermal stresses from one-sided heating should be less due to the lower energy $(2.45 \mathrm{MeV})$ neutron's effect on thermal expansion. ${ }^{39}$ Lastly, $2.45 \mathrm{MeV}$ neutrons should not induce significant swelling through helium generation. ${ }^{37,40}$ In a related issue, beryllium could have a detrimental effect on spin-polarized fuel. It has been suggested from today's research reactors that recycling of fuel off a metallic first wall surface could cause significant depolarization ${ }^{25}$. This issue must be resolved should the effect is verified.

\section{Radiation and Shielding Assessment}

The amount of Bremstrahlung radiation deposited within each material of the first wall was evaluated using Equation 13 (where power incident into a layer $\left(\mathrm{P}_{\text {in }}\right)$, power transmitted out $\left(\mathrm{P}_{\text {out }}\right)$, mass absorption coefficient ${ }^{41}\left(\mu_{\mathrm{a}} / \rho\right)$ (sometimes calculated), scrape off layer thickness $(\Delta s)$, density $(\rho)$, and total thickness of each material (x)). All $\mu_{\mathrm{a}} / \rho$ values were 
evaluated at $50 \mathrm{keV}$ and were sometimes calculated from summed weight percents of constituent element mass absorption coefficients. The neutron absorptions were similarly found through Equation 13, where the mean free paths were calculated assuming bi-level energy distributions (2/3 of macroscopic cross sections evaluated at $2.45 \mathrm{MeV}$ ). Carbon cross section and mass absorption data were used to calculate neutron and $\mathrm{X}$-ray mean free paths for the $\mathrm{C}-\mathrm{G}$ matrix composite. ${ }^{41,42}$

$$
P_{\text {out }}=P_{\text {in }}\left(\frac{a+\Delta s+x}{a+\Delta s}\right) e^{-\left(\frac{\mu_{a}}{\rho}\right) \rho x}
$$

All radiation that did not pass directly to space was absorbed by the first wall and shield structures, converted to heat, and transferred by the GHe coolant. For the inboard assembly, opacity, high temperature operation, and low density were needed for shielding the central superconductor from all Bremstrahlung and neutron radiation. Carbon-graphite was chosen to attenuate the inboard-bound neutrons, while the refractory tungsten boride $\left(\mathrm{W}_{2} \mathrm{~B}_{5}\right)$ was used to coat the shield's inboard side (to minimize mass) to completely absorb all Bremstrahlung radiation transmitted through the first wall. $\mathrm{W}_{2} \mathrm{~B}_{5}$ has high temperature $\left(\sim 2,600{ }^{\circ} \mathrm{K}\right)$ capability, lower density $\left(11 \mathrm{~g} / \mathrm{cm}^{3}\right)$, and relatively short radiation mean free paths making it an attractive shielding material. ${ }^{43}$ The results of the radiation assessment are shown in Figure 10.

A small amount of the inboard assemblybound residual neutron power, $\sim 88 \mathrm{~kW}$ and $\sim 45 \mathrm{~kW}$, was absorbed by the YBCO and its $\mathrm{C}-\mathrm{G}$ substrate structure respectively. This heat was captured and rejected by the $\mathrm{LN}_{2}$ refrigeration system at $64^{\circ} \mathrm{K}$.

\section{Inboard Assembly}

The inboard assembly consisted of radiation shielding, GHe coolant channel, superconducting YBCO central conductor magnet, $\mathrm{LN}_{2}$ refrigeration channel, Titanium alloy strengthener, slush hydrogen propellant channel, and various $\mathrm{C}-\mathrm{G}$ walls and vacuum insulation gaps (Figure 12). The radiation shielding consisted of $\sim 34 \mathrm{~cm}$ of C-G composite with $\sim 1 \mathrm{~mm}$ of $\mathrm{W}_{2} \mathrm{~B}_{5} \mathrm{x}$-ray attenuation coating. The GHe coolant channel was $3 \mathrm{~cm}$ wide and was bounded by the shielding and the $0.5 \mathrm{~cm}$ thick $\mathrm{C}-\mathrm{G}$ inboard wall. Its flow of coolant provided heat transfer for almost all of the $444 \mathrm{MW}$ of radiation power. A $1 \mathrm{~cm}$ vacuum insulation gap mitigated conduction/convection of heat from the $1,912{ }^{\circ} \mathrm{K}$ C-G wall (with a low emissivity coating) to the superconducting magnet at $65^{\circ} \mathrm{K}$. The magnet was a $0.5 \mathrm{~cm}$ thick $\mathrm{C}-\mathrm{G}$ substrate coated with $0.2 \mathrm{~cm}$ thick YBCO superconductor. A $0.5 \mathrm{~cm}$ thick layer of $\mathrm{LN}_{2}$ refrigerant adjacent to the magnet/C-G established cryogenic temperatures prior to reactor ignition and maintained those temperatures by removing residual neutron heat. Another $1 \mathrm{~cm}$ vacuum insulation gap separated this layer from the Titanium alloy strengthener. This $2.35 \mathrm{~cm}$ strengthener was a compression structure that accommodated the loads generated by the central conductor's magnetic field and the tension loads of the attached TF coils. Another vacuum insulation gap $(61 \mathrm{~cm})$ separated the Titanium alloy strengthener from the slush hydrogen propellant channel that ran along the reactor's centerline. Its thickness was merely the residual radial distance and could be used for additional shielding, coolant flow, etc. should that prove necessary. Lastly, a $2.5 \mathrm{~cm}$ radius propellant channel ran along the reactor centerline.

A cylindrical central conductor with demountable TF return leg coil design was chosen because of certain attractive features compared to discrete coil designs. ${ }^{44}$ It permitted a more efficient use of cross sectional area for superconducting current flow. The relative thinness of the YBCO magnet layer also minimized the amount of material intercepting residual neutron flux, thereby reducing the heavy, powerintensive refrigeration system required for heat rejection at low temperature. Adverse inter-coil magnetic fields

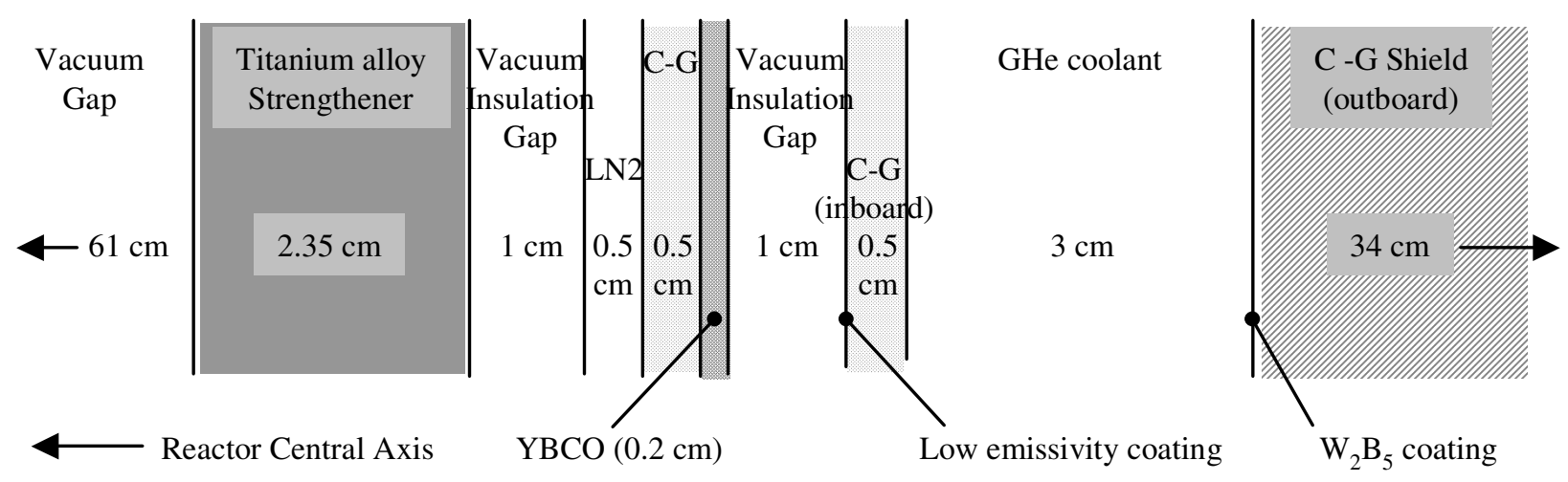

Figure 12: Inboard Assembly 
that could limit critical superconducting currents were also reduced or eliminated. In addition, on-orbit reactor servicing should be simplified by the ability to demount and replace individual TF coils if necessary. At each end of the central conductor were truncatedconical, current carrying structures that served as the transitions from the central conductor to the TF coils, where each coil was attached at both ends.

$$
-\sigma=\frac{-2 r_{o}^{2} p}{\left(r_{o}+r_{i}\right) t}
$$

The cylindrical titanium alloy strengthener, inboard of the central conductor, countered the large compression forces generated by the conductor's current. A thick-wall cylinder relation was used to estimate the strengthener's thickness (Equation 14; thickness $(\mathrm{t})$, outer and inner radius $\left(\mathrm{r}_{\mathrm{o}}\right.$ and $\left.\mathrm{r}_{\mathrm{i}}\right)$, external pressure force $(\mathrm{p})$, and compressive limit stress $(\sigma))$. The pressure was calculated through the same loads assessment algorithm used for the TF coils. The material compression stress limit was taken to be half of the tensile limit at temperature $\left(9.5 \cdot 10^{8} \mathrm{~Pa}\right)$, and yielded a required thickness $(\mathrm{t})$ of $2.35 \mathrm{~cm}$.

\section{Toroidal/Poloidal Field Coils}

A twelve toroidal field (TF) coil and seven poloidal field (PF) coil configuration was used. The coils' crossectional area was $75 \% \quad \mathrm{YBa}_{2} \mathrm{Cu}_{3} \mathrm{O}_{7}$ (YBCO) surrounded by a liquid nitrogen refrigerant, followed by an aluminum lithium casing. A summary of selected TF coil magnet parameters is given in Table 11. YBCO was chosen for its extremely high critical current density at moderate to large external magnetic fields, even at temperatures as high as $77{ }^{\circ} \mathrm{K}{ }^{45}$. Recent thin film experiments using a chemical vapor deposition process have demonstrated that the critical current density $\left(\mathrm{J}_{\text {crit }}\right)$ is largely a function of magnetic field orientation at right angles to the material c-axis. ${ }^{46}$ Critical current densities as great as 3,000 MA/m $\mathrm{m}^{2}$ were measured for samples at $\sim 65^{\circ} \mathrm{K}$ and magnetic fields of $\sim 2 \mathrm{~T}$ with such magnetic field orientations. ${ }^{46}$ In addition, irradiation experiments have measured enhancements in critical current density of up to a factor 4 for fields parallel to the c-axis and a factor of 2 increase for fields at right angles to the c-axis. These results were obtained from melt textured YBCO exposed to inelastic scattering by fast (1-2 MeV) neutrons, with fluences of 2 to $6 \cdot 10^{21} / \mathrm{m}^{2}{ }^{47,48}$ These results suggest that critical current densities of the order $12,000 \mathrm{MA} / \mathrm{m}^{2}$ might be achievable in monolithic structures in the time frame of the Discovery II. Such high densities permitted greatly reduced central coil thickness compared to Type II superconductors, minimized heat absorption of attenuated neutrons, more efficient use of central core volume for heat removal, and integration of a central propellant flow channel.

Table 11: TF Coil Characteristics

\begin{tabular}{|c|c|}
\hline Number of coils & 12 \\
\hline Superconductor material & YBCO \\
\hline Length (w/o central conductor or trans) (m) & 12.13 \\
\hline Radius (cm) & 1.8 \\
\hline Hydraulic diameter (w/ 5:1 fin ratio) (m) & 0.04 \\
\hline Cross sectional area (superconductor) $(\%)$ & 75 \\
\hline Cross sectional area (refrigerant) $(\%)$ & 20 \\
\hline Current per coil (MA) & 9.2 \\
\hline Current density $\left(\mathrm{MA} / \mathrm{m}^{2}\right)$ & 12,000 \\
\hline Toroidal magnetic field (centerline) (T) & 8.9 \\
\hline Maximum magnetic field (self at coil) $(\mathrm{T})$ & 32.3 \\
\hline Maximum magnetic field (external) (T) & 1.6 \\
\hline Operating temperature $\left({ }^{\circ} \mathrm{K}\right)$ & 65 \\
\hline Refrigerant & $\mathrm{LN}_{2}$ \\
\hline Fluence $\left(10^{23} / \mathrm{m}^{2}\right)$ & 2.25 \\
\hline Coil strengthener material & Ti-6Al-4V \\
\hline Strengthener configuration (I-beam) & $\mathrm{W}-36$ \\
\hline Maximum tensile stress $\left(65^{\circ} \mathrm{K}\right)\left(\mathrm{N} / \mathrm{m}^{2}\right)$ & $19 \cdot 10^{8}$ \\
\hline Section modulus $\left(\mathrm{m}^{3}\right)$ & 0.052 \\
\hline Force per unit length (midplane) (N/m) & $3.22 \cdot 10^{7}$ \\
\hline Force per unit length (top) (N/m) & $7.38 \cdot 10^{7}$ \\
\hline Maximum bending moment $(\mathrm{Nm})$ & $1.08 \cdot 10^{8}$ \\
\hline \multicolumn{2}{|l|}{ Total mass of $12 \mathrm{TF}$ coils } \\
\hline magnets (mt) & 1.5 \\
\hline strengtheners (mt) & 127.1 \\
\hline
\end{tabular}

The TF coil radius was determined by calculating the resultant external magnetic field from one coil on to the adjacent coil, deducing the greatest critical superconducting current permitted by that field, and then solving for the TF coil dimensions. The resultant external magnetic field was estimated through the short current element approximation (Equation 15). ${ }^{49}$ The magnetic induction (B) was thus a function only of coil current (I), the angles between the perpendicular and the effective end of the current element $\left(\theta_{1}\right.$ and $\left.\theta_{2}\right)$, the distance between adjacent TF coils at the point of closest approach $(\mathrm{y})$, and magnetic constant $\left(\mathrm{k}_{\mathrm{m}}\right)$. A total central conductor current of 110.4 MA (9.2 MA per TF coil) was required to drive the large $8.9 \mathrm{~T}$ on-axis field. A separation distance of less than one meter and an effective current element angle taken to be $45^{\circ}$ yielded an external magnetic field of $\sim 1.6 \mathrm{~T}$ at the surface of each $\mathrm{TF}$ coil where they attach to the central conductor. This became the constraint on the critical current for the superconducting material. 


$$
B=\frac{k_{m} I}{y}\left(\sin \theta_{1}+\sin \theta_{2}\right)
$$

With a $J_{\text {crit }}$ of $12,000 \mathrm{MA} / \mathrm{m}^{2}$, the total coil radius and central conductor thickness were $1.8 \mathrm{~cm}$ and $0.2 \mathrm{~cm}$ respectively. The fast $(1-2 \mathrm{MeV})$ neutron fluence was calculated to be $\sim 2 \cdot 10^{23} / \mathrm{m}^{2}$, which was two orders of magnitude greater than current observations, signaling a need for either more central conductor shielding or further experimentation to discover the upper limit to $\mathrm{J}_{\text {crit }}$ enhancing irradiation.

A load carrying assessment was made by utilizing an analytic approximation for sizing constant tension D-shape magnets for fusion reactors. ${ }^{50}$ By calculating the force per unit length $\left(f_{1}\right)$ given by Equation (16) (where radius of curvature angle $(\phi)$ and conductor radius $\left(\mathrm{c}_{\mathrm{r}}\right)$ ) then substituting into Equation (17) defining the radius of curvature $(\rho)$ of a flexible conductor under constant tension (T), Equation (18) could then be solved describing the geometric shape of the $\operatorname{coil}^{51}$ ( $\mathrm{z}$ is axis of toroidal symmetry).

$$
\begin{gathered}
f_{l}=\frac{\mu_{o} N I^{2}}{4 \pi R}\left[1+\frac{1}{N}\left(\cos \phi+\frac{R}{\rho} \ln \frac{1.284 R}{c_{r} N}\right)\right] \\
\rho_{l}=\frac{T}{f_{l}} \\
\rho_{l} \frac{d^{2} \rho}{d z^{2}}= \pm \frac{\mu_{o} N I_{c}}{2 \pi}\left[1+\left(\frac{d \rho}{d z}\right)^{2}\right]^{3 / 2}
\end{gathered}
$$

The geometry of the D-shaped TF coils were determined by curve fitted polynomials to the solutions of Equations (16) through (18) of the form of Equation (19). Equation (19) was parameterized for four essential coil dimensions $\left(\mathrm{P}_{\mathrm{i}}\right)$, where the outermost coil location $\left(\rho_{2} / R_{2}\right)$ for the $\phi=0$ position was set as the independent variable.

$$
\frac{P_{i}}{R_{2}}=\sum_{j=0}^{4} A_{j}\left(\frac{\rho_{2}}{R_{2}}\right)^{j}
$$

The radial build resulting from Equation (19) produced a design with an excessive $\mathrm{R}_{1}$ (for the $\mathrm{H}_{2}$ propellant channel and central strengtheners) and insufficient volume for the cylindrical C-G shield. As a result, $\mathrm{R}_{1}$ was decreased and the other primary dimensions were adjusted accordingly to maintain the general D shape. The tension loads, however, were recalculated for the actual TF coil positions for consistency, even though this produced greater loads and thus required more massive strengtheners. In addition, both vertical dimensions had to be increased $50 \%$ to accommodate the large elongation (3.0) of the reactor design. The original algorithm (Equation (16)) was not found to be conducive to highly elongated toroids. The result of this approach to sizing and placing TF coils may not have been rigorously correct, but was reasonably self consistent and accounted for the primary current-driven tension forces on the coils.

The results from this algorithm were also used to estimate the compression loads exerted by the central conductor on the cylindrical and truncated conical transition strengtheners. The force per unit lengths calculated were taken as compression values along both titanium structures. The thickness of the truncated conical transition strengtheners was determined by straight-line transitioning the compression loads within the central conductor strengthener to the bending loads within the I-beam strengtheners of the TF coils.

The high current (9.2 MA) carried in the coils that produced the tension loads necessitated strengtheners made from the highest yield strength material suitable for low temperature applications. The Titanium alloy, Ti-6Al-4V, was selected for its high yield strength $\left(19 \cdot 10^{8} \mathrm{~N} / \mathrm{m}^{2}\right.$, annealed or solution treated) at cryogenic temperatures ${ }^{52}$. The Ti-6Al-4V cross section design chosen was that of an AISC W-36 shape I-beam (Figure 13), mounted onto the outboard side of each TF coil. The W-36 is one of the largest standard I-beams available and was used due its large section modulus $\left(0.052 \mathrm{~m}^{3}\right){ }^{53}$ To approximate the distributed tension loading, each strengthener beam was envisioned as a curved support, matching each elliptical TF coil, and was designed for the maximum bending moment (evaluated at the reactor mid-plane). The tension forces calculated by the algorithm were averaged to create a uniform load distribution, which was counteracted by structural supports from the seven PF coils and the two truncated conical transition strengtheners (supporting the beam ends) from the central conductor. The resulting bending moment relation was derived to be (Equation 20, where $f_{\text {outer }}$ and $f_{\text {inner }}$ were the midplane and top tension force per unit lengths respectively, and 1 was the total length of the I-beam). The maximum bending moment was $\sim 10^{8} \quad \mathrm{~N}-\mathrm{m}$. Exploration of less massive strengthener concepts was deferred. The 12 TF coil mass was $\sim 129 \mathrm{mt}$ (Table 12). 


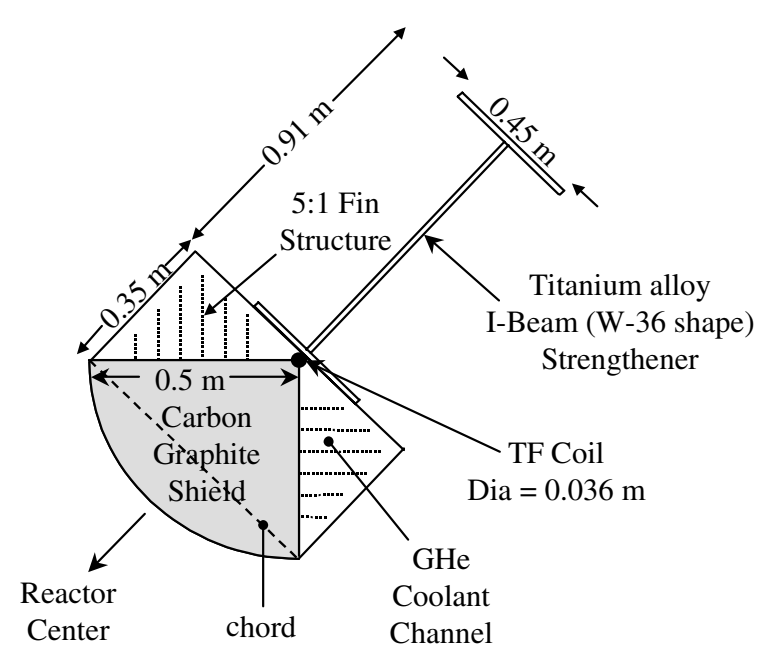

Figure 13: TF Coil Cross Section

$$
M=\left(f_{\text {outer }}+f_{\text {inner }}\right) l^{2} / 144
$$

Minimal analysis was done on the PF coils and their additional function to serve as cross bracing for the TF coils. The attractive Ampere's law-driven forces between the TF coils were calculated but were found to be less than $6 \%$ of those of the tension loads. Cross bracing for these as well as the "overturning" forces were assumed to be accounted for by the design, including the additional mass penalty. Based on ITER and similar test reactors, seven PF coils were assumed, with coil/shielding designs and masses (including crossbracing) scaled to $60 \%$ of the TF coils as recommended by similar advanced small aspect ratio reactor studies. ${ }^{54}$ The cross sectional makeup was assumed to be identical to the TF coils/strengtheners, but without C-G shields, since the TF coil shields would shadow much of the PF coils. The total $7 \mathrm{PF}$ coil/cross-bracing mass was $77 \mathrm{mt}$.

Table 12: Fusion Reactor Mass Properties

\begin{tabular}{lr}
\hline First wall & 14 \\
Toroidal field coils (12) & 129 \\
Poloidal field coils (7) and cross bracing & 77 \\
Neutron and radiation shielding & 90 \\
- & --- \\
Total (mt) & 310 \\
\hline
\end{tabular}

The outboard C-G radiation shield was a $1 / 4$ sector cylinder design so that TF coils would be protected from neutron (and Bremsstrahlung) radiation from within a $90^{\circ}$ angle centered on the reactor central axis. (Figure 13). This design minimized shield mass while also permitted neutrons that were not impacting the coils to pass directly to space. A minimum $50 \mathrm{~cm}$ blanket thickness was recommended for $\mathrm{D}^{3} \mathrm{He}$ fueled reactors. ${ }^{55,56}$ The less than $4 \pi$ shielding design meant that human operations could not be conducted around the reactor without protection while the reactor was operating. However, the convergence of the shields at the forward (crew module-facing) pole of the reactor provided an estimated $60^{\circ}$ shadow shield. This was sufficient protection for limited human operations around the vehicle aft and protracting systems (i.e. propellant tankage and radiators). An equivalent amount of shielding was removed in the corresponding location aft of the reactor since the largely skeletal divertor/ magnetic nozzle coils had their own shielding. The total C-G shield mass was $90 \mathrm{mt}$.

\section{Heat Transfer}

A heat transfer analysis was performed by first assessing the amount of radiation that passed directly to space without intercepting structure. The reactor's physical layout and structural materials were designed to maximize this direct flow. For the radiation that was intercepted by structure and thermalized, a GHe coolant system was designed to efficiently transfer heat at high temperature to minimize radiator mass.

A geometric analysis was made of the amount of neutron and Bremsstrahlung power that was intercepted by the inboard reactor assembly and the $\mathrm{TF} / \mathrm{PF}$ coils. These quantities were then subtracted from the total radiation powers to solve for the amounts that could pass freely to space. For simplicity, all radiation fluxes were assumed to be normal to the surface of the first wall. Equation 21 was the resulting relation to calculate the fraction of surface area facing inboard (i.e. all surface element normals with a component towards the reactor centerline) of a torus $\left(\mathrm{S}_{\mathrm{in} / \text { total }}\right)$. For the Discovery II, $\mathrm{S}_{\mathrm{in} / \text { total }}=3 / 8$. For simplicity and conservativeness, it was assumed that all inboard radiation fluxes were completely absorbed by the inboard assembly.

$$
S_{\text {in/total }}=\frac{R-a / 2}{2 R}
$$

The fractions of surface area outboard of the reactor that intercepted radiation were calculated separately for the neutron and Bremsstrahlung fluxes. The fraction of neutrons absorbed was based only on the projected reactor facing area of the titanium strengtheners behind the $\mathrm{C}-\mathrm{G}$ shield. The fraction of Bremsstrahlung absorbed was based on the entire projected reactor facing area of the $\mathrm{C}-\mathrm{G}$ radiation shield, since the soft $\mathrm{x}$-rays would be totally absorbed by the $\mathrm{C}-\mathrm{G}$ and $\mathrm{W}_{2} \mathrm{~B}_{5}$ material. The results showed that the fractions of outboard bound neutron and 
Bremsstrahlung powers intercepted by the TF coils $\left(\mathrm{S}_{\text {intercp/out }}\right)$ were functions of $\mathrm{R}$, a, and the projected area based on the TF coil chord (s) (titanium strengthener flange for neutron radiation and $\mathrm{C}-\mathrm{G}$ chord for Bremsstrahlung radiation). (Equation 22) The PF coils were not analyzed separately. The intercepted radiation fractions were assumed to be $10 \%$ of the TF values.

$$
S_{\text {intercp/out }}=\frac{6 s}{\pi(R+a / 2)}
$$

Figure 10 illustrates that $46 \%$ of the outboard bound Bremsstrahlung power $(27 \%$ of the total generated) and $71 \%$ of the outboard bound neutron power (34\% of the total generated) was radiated directly to space without need of a heat rejection system. These values, when combined with the synchrotron loss fraction, indicate that $35 \%$ of the total radiation power may be radiated directly to space without need of a heat rejection system.

A GHe coolant system was created to transfer the remaining radiation (65\% of total produced) that was thermalized to the heat rejection system (radiators) and power conversion system at high temperature. GHe was chosen so that it could be used directly as the working fluid for the Brayton power conversion equipment due to its chemical inertness. High-pressure (7.5 $\mathrm{MPa}$ ) operation was required, however, due helium's low heat capacity. Three commingled coolant loops transferred $\sim 92 \%$ of the 1,215 MWt of thermal heat from the first wall, inboard assembly, and TF/PF coils to the radiators. Only $96 \mathrm{MWt}$ was bled off of the loops and sent to the power conversion cycle. The loops all had reactor exit and entrance temperatures $\left(\mathrm{T}_{\text {hot }}\right.$ and $\mathrm{T}_{\text {cold }}$ ) of 1,700 and $997{ }^{\circ} \mathrm{K}$ respectively. The total mass flow rate of the three loops was $333 \mathrm{~kg} / \mathrm{sec}$.

The GHe coolant was sent to a lightweight carbon-carbon, parallel duct heat pipe radiator panel array. The radiator operated at an average wall temperature of $1,325{ }^{\circ} \mathrm{K}$ and had a total radiating surface area of $4,070 \mathrm{~m}^{2}$. Using a planar configuration, this area was arranged as eight panels, rejecting heat from both sides. Each panel was $10 \mathrm{~m}$ wide and $25 \mathrm{~m}$ long; the length limited by launch packaging, deployment, and rigidity imposed constraints. The radiator was adjacent to the lower temperature power conversion radiator and the cryotankage, in the interest of minimizing coolant line lengths and concomitant losses for most of the mass flow. The amount of thermal radiation heating to the cryotankage was deferred to later study. The radiator mass was $4.5 \mathrm{mt}$. A lowpressure ratio fan, operating at 9,000 rpm circulated the $\mathrm{GHe}$ at $\sim 306 \mathrm{~kg} / \mathrm{sec}$. The mass of the drive motor and fan were 4 and $3 \mathrm{mt}$ respectively. The required fan power for the coolant loop was 23.6 MW (with 92\% efficient fans) with an assumed pressure ratio of 1.035 required for circulation of the coolant flow against a system pressure drop (3.5\%) (Table 13).

\section{Table 13: Coolant System Characteristics}

\begin{tabular}{lc}
\hline Coolant & $\mathrm{GHe}$ \\
Pressure $(\mathrm{MPa})$ & 7.5 \\
Temperature $($ hot gas $)\left({ }^{\circ} \mathrm{K}\right)$ & 1,700 \\
Temperature $($ cold gas $)\left({ }^{\circ} \mathrm{K}\right)$ & 997 \\
Mass flow rate $(\mathrm{kg} / \mathrm{sec})$ & 306.4 \\
Power transferred $\left(\mathrm{MW}_{\mathrm{t}}\right)$ & 1,119 \\
Radiator type & $\mathrm{C}-\mathrm{C}$ heatpipe \\
Average temperature $\left({ }^{\circ} \mathrm{K}\right)$ & 1,325 \\
Total area $\left(\mathrm{m}^{2}\right)$ & 4,070 \\
Motor type & 3 phase AC, $600 \mathrm{~Hz}$ \\
Fan efficiency $(\%)$ & 92 \\
System pressure loss $(\%)$ & 3.5 \\
Fan type & axial, single stage \\
Fan speed $(\mathrm{rpm})$ & 9,000 \\
Fan power $(\mathrm{MWe})$ & 23.6 \\
\hline
\end{tabular}

The first wall loop transferred $405 \mathrm{MWt}$ (including $96 \mathrm{MWt}$ to the Brayton power conversion system) at low flow speed $(\mathrm{M \#}=0.02)$. The double-hull design of the $\mathrm{C}-\mathrm{G}$ first wall was created primarily to dissipate the significant thermalized synchrotron radiation (268 MWt) on the plasma-facing surface. The average temperature difference between the wall and coolant film temperature was $477^{\circ} \mathrm{K}$, permitting good heat transfer. The heat transfer coefficient $\left(h_{c}\right)$ was calculated to be $1,804 \mathrm{~W} /\left(\mathrm{m}^{2}{ }^{\circ} \mathrm{K}\right)$ using the long duct, turbulent flow relation (a function of thermal conductivity $\left(\mathrm{k}_{\mathrm{f}}\right)$, hydraulic diameter $\left(\mathrm{D}_{\mathrm{H}}\right)$, Nusselt number $\left(\mathrm{N}_{\mathrm{u}}\right)$, Reynolds number $\left(\mathrm{R}_{\mathrm{e}}\right)$, and Prandtl number $\left(\mathrm{P}_{\mathrm{r}}\right)$ ) (Equation 23) ${ }^{57}$. Table 14 summarizes the heat transfer data for all three coolant loops.

$$
\overline{h_{c}}=\frac{k_{f}}{D_{H}}\left(N_{u}\right)=\frac{k_{f}}{D_{H}} 0.023\left(R_{e}\right)^{0.8}\left(P_{r}\right)^{1 / 3}
$$

The inboard assembly/central conductor loop transferred $444 \mathrm{MWt}$ of thermalized neutron and Bremsstrahlung power at high flow speed $(\mathrm{M \#}=0.16)$. The cylindrical design of the inboard heat transfer coolant channel was bounded by the inner edge of the $\mathrm{W}_{2} \mathrm{~B}_{5}$ coated $\mathrm{C}-\mathrm{G}$ shield and an inboard $\mathrm{C}-\mathrm{G}$ wall. This design was created primarily to dissipate the significant thermalized Bremstrahlung radiation (357 MWt) largely in the $\mathrm{W}_{2} \mathrm{~B}_{5}$ layer. Of the three coolant loops, this loop represented the greatest challenge due to the minimal volume available to transfer a prodigious amount of heat. This was accomplished by a high velocity 
$\sim 340 \mathrm{~m} / \mathrm{sec}$ flow and a very high heat transfer coefficient $9,190 \mathrm{~W} /\left(\mathrm{m}^{2}{ }^{\circ} \mathrm{K}\right)$; incorporating a $2: 1$ ratio thin-fin, shell-like structure to increase the hot surface area. The average temperature difference between the wall and coolant film temperature was $562{ }^{\circ} \mathrm{K}$.

Table 14: Heat Transfer Characteristics (Reactor Side)

\begin{tabular}{lccc}
\hline Coolant loop & $1^{\text {st }}$ Wall & Inboard & TF/PF \\
\hline Coolant & GHe & same & same \\
Pressure (MPa) & 7.5 & same & same \\
Temperature (hot gas) ${ }^{\circ} \mathrm{K}$ & 1,700 & same & same \\
Temperature (cold gas) ${ }^{\circ} \mathrm{K}$ & 1,000 & same & same \\
Temperature (wall) ${ }^{\circ} \mathrm{K}$ & 1,824 & 1,912 & 1,759 \\
Mass flow rate $(\mathrm{kg} / \mathrm{sec})$ & 111 & 122 & 100 \\
Velocity (m/sec) & 44.6 & 341.3 & 25.0 \\
Mach number & 0.02 & 0.16 & 0.01 \\
Reynolds number & 124,663 & 953,987 & 48,292 \\
Nusselt number & 238 & 1212 & 111 \\
Heat trans coef $\left(\mathrm{W} / \mathrm{m}^{2}{ }^{\circ} \mathrm{K}\right)$ & 1,804 & 9,190 & 1,224 \\
Heat transferred $(\mathrm{MW})$ & $309+96$ & 444 & 366 \\
\hline
\end{tabular}

The TF/PF coil loop transferred $366 \mathrm{MWt}$ of thermalized neutron and Bremsstrahlung power at very low flow speed $(\mathrm{M \#}=0.01 ; 25 \mathrm{~m} / \mathrm{sec})$. The $\mathrm{TF}$ coil coolant channel was bounded by the outer edge of the $\mathrm{W}_{2} \mathrm{~B}_{5}$ coated $\mathrm{C}-\mathrm{G}$ shield and an outboard $\mathrm{C}-\mathrm{G}$ wall. This design was similar to the inboard assembly, though without the imperative of attenuating all radiation. Most of the thermalized radiation was Bremstrahlung (323 MWt) largely within the $\mathrm{W}_{2} \mathrm{~B}_{5}$ layer. Of the three coolant loops, this loop was the least demanding due to the ample coolant volume available for heat transfer. The heat transfer coefficient was $1,224 \mathrm{~W} /\left(\mathrm{m}^{2}{ }^{\circ} \mathrm{K}\right)$; incorporating a thin-fin, shell-like structure that increased hot surface area by a factor of $5: 1$. The average temperature difference between the wall and coolant film temperature was $409^{\circ} \mathrm{K}$.

\section{Coaxial Helicity Ejection}

Coaxial Helicity Ejection (CHE) was used to permit control of the plasma current and "the amount of plasma kinetic power exhausted into the magnetic nozzle (plasma energy and particle loss rate from the core)". ${ }^{30}$ Magnetic helicity (approximately equal to the product of toroidal and poloidal magnetic fluxes) can be injected into a plasma by maintaining a voltage across the divertor while injecting current into its channel. The inverse process, ejecting helicity (which normally occurs on shutdown), can theoretically provide a means for extracting power out of the plasma. CHE (in conjunction with bootstrap current overdrive) is anticipated to provide a direct means for controlling both the exhausted transport power into the magnetic nozzle as well as the plasma current. This should facilitate propulsion operations while still sustaining reactor conditions. For example, increased jet power could be achieved by increased fusion power (via increased fueling rate), which would increase bootstrap current. The increased bootstrap current would permit increased exhausted transport power through $\mathrm{CHE}{ }^{30}$ Precisely how $\mathrm{CHE}$ would operate in conjunction with bootstrap current overdrive and reactor fueling is the focus of the theoretical and experimental work.

To examine the potential for CHE, planning has commenced at DOE's Princeton Plasma Physics Lab (PPPL) in cooperation with NASA GRC. A series of small, proof of concept experiments on the newly completed National Spherical Torus Experiment (NSTX) reactor are currently being evaluated. They are intended to provide essential experimental data to establish the scientific theory and assess the feasibility of CHE. The initial experiment will focus on how plasma near the divertor plate can be ejected, including measurements of changes in local magnetic field, movements of current towards the scrape off layer, and establishing plasma state conditions. Validated theoretical models and experiment diagnostics will then be used in follow-on tests mutually beneficial to both space propulsion applications and terrestrial fusion power research. If successful, CHE operation will be integrated with the recently initiated magnetic nozzle proof of concept experiment.

\section{Divertor}

By diverting plasma in the scrape off layer via CHE into the propulsion system, fusion energy served to directly heat propulsive mass without first going through intermediate, material heat flux-limited, lowefficiency power conversion equipment. An efficient divertor thus enabled a direct thrust approach to space propulsion by transferring high energy charged particles out of the reactor and into the reservoir of the magnetic nozzle. As will be shown, $\sim 98 \%$ of the particles exiting the reactor were un-reacted $\mathrm{D}$ and ${ }^{3} \mathrm{He}$ ions and electrons. The remaining particle densities (neutral impurities, ${ }^{4} \mathrm{He}$, and $\mathrm{p}$ ash) had to be kept low to maintain good confinement and propulsion performance. As these particles cooled and migrated to the outer field lines in the scrape-off layer, they eventually were pulled into the divertor and out of the reactor. $\mathrm{T}$ and $\mathrm{n}$ in the scrape-off were calculated to be $\mathrm{T}<4 \mathrm{keV}$, and $\mathrm{n}_{\mathrm{i}}<2 \cdot 10^{20} / \mathrm{m}^{3}$ (Figure 14).

The open divertor design was only conceptual. Basic plasma, MHD, and related fusion science analysis remain to be performed through the proposed $\mathrm{CHE}$ 

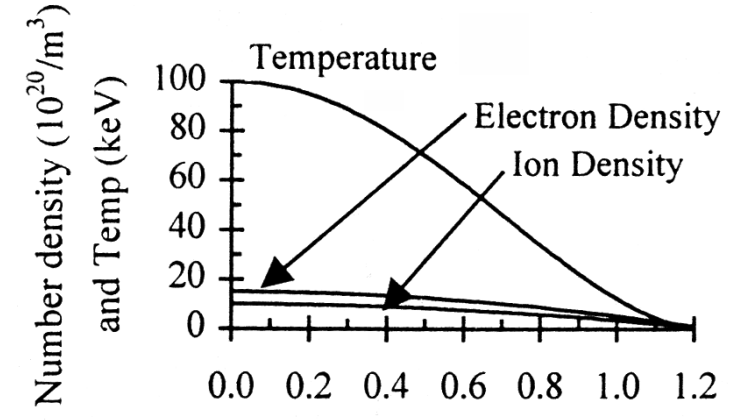

\section{Minor Radius (m)}

\section{Figure 14: Plasma Temperature and Number Density Radial Profiles}

experiment and related efforts. The divertor's geometric design (single null, located in the reactor aft) was patterned somewhat after ITER's. ${ }^{58}$ Three PF coil-like divertor coils (carrying reduced current through a smaller coil radius) were within the reactor's minor radius, between the TF coils to divert the magnetic field outside of the reactor. The field lines exited and reentered the reactor through the double annulus, carrying the exhaust plasma to the magnetic nozzle. The estimated combined width of the double annulus and middle coil was $0.5 \mathrm{~m}$, scaled from ITER geometry. ${ }^{59}$ The divertor coils' plasma facing material had to withstand high temperatures and high erosion rates. Carbon fiber composites, such as Aerolor A05, ${ }^{60}$ are likely candidates to tolerate surface temperatures up to $1,500{ }^{\circ} \mathrm{C}$ with peak heat loads of at least $5 \mathrm{MW} / \mathrm{m}^{2}$ and transient heat loads potentially as great as $20 \mathrm{MW} / \mathrm{m}^{2}{ }^{58}$ Total mass of all three coils and other components (structure, power lines, etc.) was set to $3 \mathrm{mt}$. (see Table 15)

Table 15: Divertor Characteristics

\begin{tabular}{lc}
\hline Configuration & Open, single null \\
Structural material & TZM \\
Plasma facing material & Aerolor A05 \\
Number of coils & 3 \\
Plasma temperature $(\mathrm{keV})$ & $<4$ \\
Plasma density $\left(10^{20} / \mathrm{m}^{3}\right)$ & $<2$ \\
Annulus width $(\mathrm{m})$ & 0.5 \\
Mass $(\mathrm{mt})$ & 3 \\
\hline
\end{tabular}

The divertor's operational concept was fundamentally different than ITER's or any of those used in today's experimental reactors. Today's reactors are not designed to exhaust vast quantities of transport power, operate in steady state, accommodate immense wall loading without assistance of radiation from injected inert gas, or exhaust plasma without collision with a target. In addition, today's divertor structural designs and resulting masses are driven by requirements that are not expected to be a primary issue with a space propulsion concept. ITER's divertor consists of 60 cassettes weighing $25 \mathrm{mt}$ each. ${ }^{58,60,61}$ Their mass is attributed to both withstanding the immense structural loadings initiated by disruptions and also shielding the vacuum vessel from neutron radiation. ${ }^{58}$ It was assumed that steady state operation of the reactor concept would at least minimize if not eliminate disruptions altogether. And since there will be no vacuum vessel, the other reason was mute.

The divertor, therefore, was envisioned as a series of structures fabricated from a molybdenum alloy know for its suitability for very high temperature applications such as radiation shields ("TZM", Mo0.5 Ti-0.1Zr) covered with Aerolor A05 plasma facing material. The coils were assumed to be the same crosssectional design as the TF coils, although at a much lower current density. Since the divertor coils were inboard of the TF coils, they must be designed be of sufficient field strength to extract plasma scrape off without having a detrimental affect on the primary field and the fusion process. In addition, the calculated plasma density in the divertor was two orders of magnitude lower than that required in the downstream reservoir. In order to prevent this significant adverse gradient from reversing the flow, some plasma pumping method $^{62}$ must be employed. Resolution of both issues is mandatory for successful overall concept.

\section{MAGNETIC NOZZLE}

\section{Overview}

The conversion of the reactor's transport power into directed thrust was accomplished in two steps by the magnetic nozzle. In the first step, the nozzle mixed high enthalpy transport plasma from the divertor with the injected hydrogen propellant in order to reduce the excessive temperature and increase total charged propellant mass flow. In the second step, it converted the propellant enthalpy into directed thrust by accelerating the flow through converging/diverging magnetic field lines. In addition, its magnetic field prevented the high temperature plasma from coming in contact with the nozzle's coils and structural members that make up the thrust chamber. Thus for a fully ionized flow, the lines of magnetic flux also served as the containment device, minimizing heat transfer loses and the need for actively cooled structure. 
The $\mathrm{I}_{\mathrm{sp}}$ 's of 20,000 to $50,000 \mathrm{lb}_{\mathrm{f}} \mathrm{sec} / \mathrm{lb}_{\mathrm{m}}$ and corresponding $\alpha$ 's required for multi-month travel to the outer planets required ion reservoir temperatures $\left(\mathrm{T}_{\text {res }}\right)$ of 100's eV. As was discussed, the too great temperature and too small number density plasma layers that entered the divertor had to be adjusted prior to acceleration through the nozzle so as to produce the mission appropriate $I_{\text {sp. }}$. This was accomplished by heating/ionizing slush hydrogen thrust augmentation propellant by the escaping reactor plasma. This then produced the desired values of bulk plasma temperature (thus $I_{\mathrm{sp}}$ ) and mass flow rate (thus thrust-to-weight). The slush hydrogen propellant was warmed with residual heat from absorbed neutron and Bremsstrahlung radiation by its pass through the reactor centerline. Although the propellant warming was beneficial, the primary reason for channeling the propellant along the centerline was to align the bulk $(\sim 98 \%)$ of the propulsive mass flow velocity and downstream magnetic nozzle thrust vectors. The propellant was then injected into the reservoir along the nozzle centerline.

A conception of a magnetic nozzle is illustrated in Figure 15. The "reservoir" of the magnetic nozzle was somewhat analogous to a conventional liquid chemical rocket engine's combustion chamber. Adjacent to the reactor's divertor, it consisted of two small radius superconducting coils of the same design and materials as the TF coils. Forming an "effective" $10 \mathrm{~cm}$ radius solenoid, they provided the meridional magnetic field to confine the converging propellant and reactor plasma streams until their temperatures equilibrated. The reservoir was in large part a "virtual" chamber due to its mostly skeletal design where magnetic field lines defined the flow boundary for charged particles passing through. This design minimized mass and heating concerns but also placed a premium on rapid, effective ionization and enthalpy

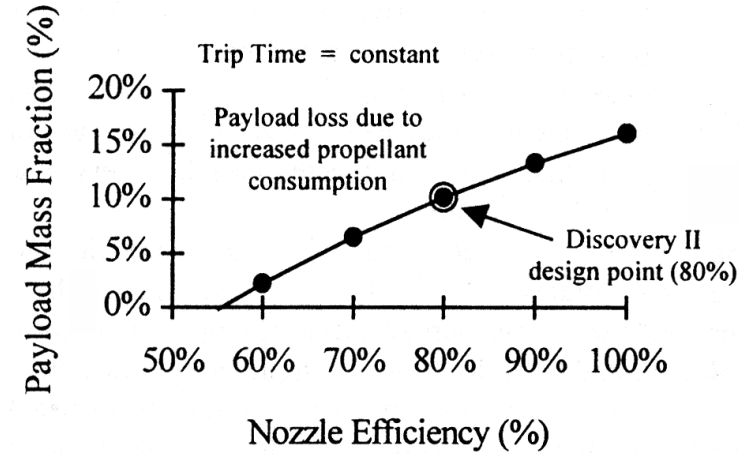

Figure 16: Efficiency vs. Payload Ratio

equilibration (i.e. neutrals were lost through the sides). The second small radius coil also constituted the "throat" for the nozzle, where choked flow (sonic) conditions existed. An arbitrarily larger radius third coil formed the downstream, diverging section and provided additional curvature to the magnetic field. The entire length of the assembly was somewhat arbitrarily set to $12 \mathrm{~m}$ with a total mass estimated at $3 \mathrm{mt}$. The nozzle coils were considerably less massive than the TF/PF coils due to negligible neutron radiation, significantly lower required magnetic field (thus lower coil current and structural loads), and lightweight Al-GrEp composite structure. The entire layout, including number of coils, geometric and magnetic field curvature, was largely notional and was intended to foster discussion, analysis, and experimentation into its salient aspects. Until mature design concepts with experimental data are available, magnetic nozzle designs, operations, and $\eta_{\mathrm{J}}$ for fusion propulsion will be largely speculative.

Much of the potential jet power could have been lost unless the internal energy was efficiently

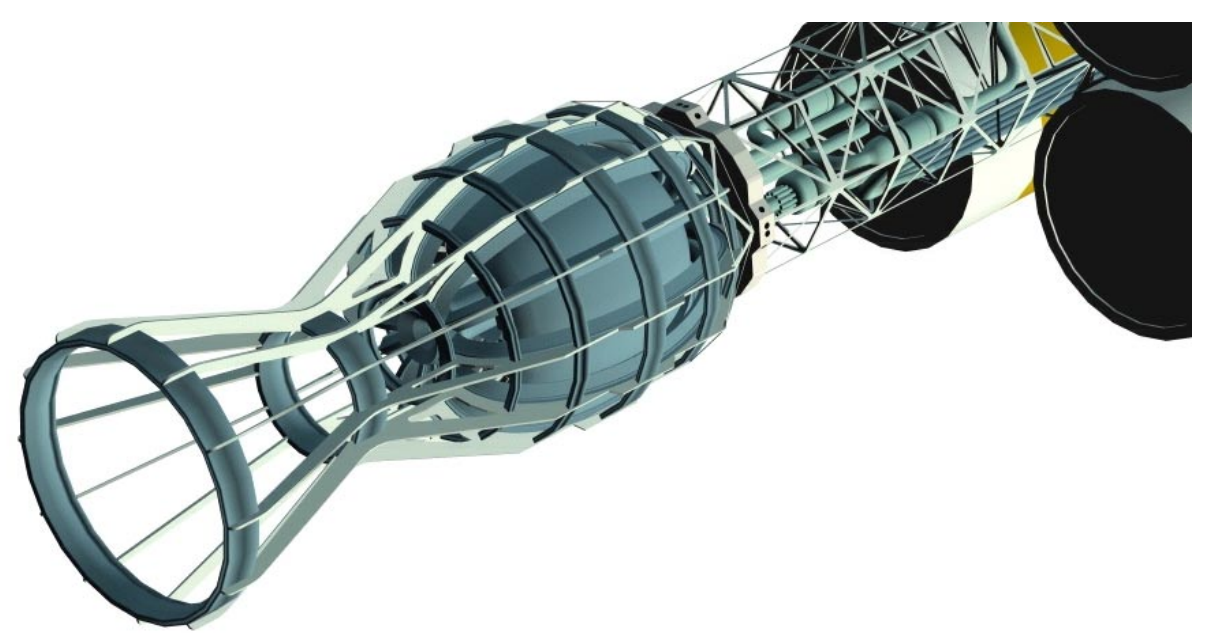

Figure 15: Magnetic Nozzle, Spherical Torus Reactor, and Brayton Power Conversion 
converted into accelerated axial flow. Estimates of the relative importance of $\eta_{J}$ on piloted interplanetary travel have been reported ${ }^{4}$. It has been shown that low $\eta_{J}$ is particularly detrimental to payload mass fraction, since decreased $\eta_{J}$ (at constant thrust) significantly increased propellant consumption. Figure 16 illustrates the profound effect of decreased $\eta_{\mathrm{J}}$ on payload mass fraction for the Discovery II. At $\eta_{\mathrm{j}}$ less than $55 \%$, the payload mass ratio vanished, underscoring the necessity of an efficient propulsion system even for otherwise respectable power supplying reactor concepts. Some theoretical analysis projecting and defining requirements for efficient magnetic nozzle operation are available, however much critical theory development is yet to be done.

\section{Theory Development}

Theory development was initiated at the Los Alamos National Lab (LANL) to support certain critical aspects of a new experiment at Ohio State University (OSU) and the vehicle design effort at NASA GRC. The theory development is focused on characterizing the necessary plasma conditions for efficient propulsion operation. Efficient propulsion will require axially symmetric plasma flow, sufficient containment by the magnetic field to minimize transport losses across field lines, prevention of plasma/structure contact, and successful detachment from field lines upon nozzle exit. Certain gradient-driven micro-instabilities are expected to negatively impact flow symmetry and instigate micro-turbulent, time-dependent fluctuations in the electric and magnetic fields. ${ }^{63}$ These fluctuations could cause diffusive scattering of plasma into the plasmafield line layer (the "current layer"). The saturation level of the fluctuations will determine the "anomalous resistivity", which then will determine the amount of mixing between the plasma and the field within the current layer. Sufficient mixing could adversely impact the flow process by precipitating a "detachment problem" for the plasma as it exits the nozzle. ${ }^{63}$ Inefficient detachment of plasma exhaust from field lines could significantly reduce propulsive thrust. This problem lacks sufficient theoretical and experimental definition and is a primary obstacle in the development of a practical fusion propulsion system.

Related theory defining these processes exists but has not been applied to MHD codes in sufficient fidelity to support space propulsion applications such as the Discovery II. Furthermore, sparse experimental data to substantiate the theory compels the current experimental effort at OSU.

\section{Experiment and Simulations}

Magnetic nozzle experimentation applicable to fusion-class propulsion was recently initiated ${ }^{64}$ by a team at Ohio State University (OSU) in conjunction with the theory development at LANL and vehicle design at NASA GRC. A series of three small, proof of concept experiments are focused on providing key experimental data for GW jet power level, quasi-steady plasma flows. The first of these experiments will measure fundamental physical parameters associated with generating and accelerating such plasmas. Of primary importance is obtaining experimental data with correct scaling of dimensionless variables (magnetic Reynolds number, current layer characteristic scale lengths, etc.) to permit extrapolation into the regime of a full scale Discovery II. The OSU facility provides an almost unique capability in this manner. Measurements will be made of plasma number density (thus mass flow rate) and velocity distribution data as functions of axial and radial position downstream of the throat. By integrating these quantities, $\mathrm{P}_{\text {jet }}$ and $\mathrm{F}$ can be calculated, inferring a value for $\eta_{\mathrm{J}}$. A variety of operating parameters should be investigated, such as reservoir temperature $\left(\mathrm{T}_{\text {res }}\right)$, density $\left(\rho_{\text {res }}\right)$, magnetic field strength $\left(\mathrm{B}_{\mathrm{res}}\right)$, choice of propellants, and others. This data will serve to test and direct LANL's theoretical work governing the nature of the plasma's current layer and its overall effect on flow conditions. Experimental and theoretical work is planned to proceed in parallel.

A 1.6 MJ capacitor bank with a nominal discharge pulse length of $1.6 \mathrm{msec}$ powers the OSU facility. ${ }^{65,66,67}$ An existing inverse-pinch switch has been modified to serve as the magnetoplasmadynamic (MPD) source test article. Along with a solenoid that will serve as a rudimentary magnetic nozzle, the assembly will be the test bed powered by the capacitor bank. Ongoing analysis will identify the required hardware modifications to the facility (such as MPD switch/magnetic nozzle dimensions), limiting operating parameters (magnetic field strength, maximum allowable stresses, etc.), and required diagnostics. Initially using helium (eventually hydrogen) ions to mimic the exhaust plasma, these experiments will enable estimation of a nozzle's performance that will be correlated to a fusion propulsion system. Further experimentation will include designing and testing a sub-scale magnetic nozzle similar to the one used for this concept, as well as designing and testing propellant injection for thrust augmentation.

Initial effort at OSU has focused primarily on analytic modeling of the test articles within a high 
fidelity $2 \frac{1}{2} \mathrm{D}$, time dependent, magnetohydrodynamic (MHD) simulation to prepare for the experiment. Initial simulations have indicated that the OSU test facility will be capable of accelerating plasma up to specific impulses of $20,000 \mathrm{lb}_{\mathrm{f}} \mathrm{sec} / \mathrm{lb}_{\mathrm{m}}$ for a duration sufficient to measure plasma state variables and physical phenomena in a comparable regime of fusion space propulsion. ${ }^{65}$ Until experimental data is available to correlate theoretical models, the performance of direct fusion propulsion systems will be largely conjecture.

\section{Magnetic Nozzle Flow and Performance Model}

Theoretical models of how a magnetic nozzle would operate and perform, as well as the primary obstacles anticipated in its development, were the subjects of a lengthy analysis. ${ }^{68}$ Idealized 1-D, MHDmodified fluid dynamic equations for a magnetic nozzle with an applied meridional magnetic field were derived in that work. Various simplifying assumptions were made such as neglecting charged particle-neutral collisions, assuming a fluid (as opposed to a kinetic) model, and neglecting various loss mechanisms such as transport, radiative, resistive, and Hall effect. A simple, single turn coil was assumed to form the throat of the magnetic nozzle. Using this approach, along with other supporting analysis, ${ }^{69,70,71}$ the flow conditions at key points through the nozzle were estimated. Isentropic flow was assumed throughout, with an arbitrary nozzle efficiency of $80 \%$ assumed (see Figure 16). For simplicity, it was assumed that the $20 \%$ nozzle loss took place at propellant-fuel enthalpy transfer.

The required downstream conditions for the exiting flow $\left(\eta_{\mathrm{J}} \alpha=6.9 \mathrm{~kW} / \mathrm{kg}\right.$ and $\mathrm{I}_{\mathrm{sp}}=35,435 \mathrm{lb}_{\mathrm{f}}$ $\mathrm{sec} / \mathrm{lb}_{\mathrm{m}}$ for the Jupiter mission) were used to initiate the flow calculations. By working back up through the nozzle, the required fuel entry conditions from the divertor were found. These conditions were required to match the transport power available from the reactor. The 1-D model revealed that the propellant exit velocity $\left(\mathrm{V}_{\mathrm{ex}}\right)$ was approximately twice the throat (sonic) velocity $\left(\mathrm{V}_{\text {throat }}\right)$, shown in Equation (24). ${ }^{68}$

$$
I_{s p}=\frac{V_{e x}}{g_{c}}=\frac{2 V_{\text {throat }}}{g_{c}}
$$

The related throat state variables (where $T_{\text {throat }}, \rho_{\text {throat }}$, $(\mathrm{PR})_{\text {throat }}, \mathrm{B}_{\text {throat }}$, and $\mathrm{A}_{\text {throat }}$ are the temperature, density, pressure, magnetic field, and cross-sectional area in the throat, and $\mathrm{R}$ is the gas constant for monatomic hydrogen (1.0078 amu)) are given by Equations 25-28:

$$
\begin{aligned}
& T_{\text {throat }}=\frac{V_{\text {throat }}^{2}}{\gamma R} \\
&(P R)_{\text {throat }}=\frac{F}{2 \gamma A_{\text {throat }}} \\
& \rho_{\text {throat }}=\frac{(P R)_{\text {throat }}}{R T_{\text {throat }}} \\
& B_{\text {throat }}=\sqrt{2 \mu_{o}(P R)_{\text {throat }}} \quad(\beta=1)
\end{aligned}
$$

The magnetic field lines created an "effective" $10 \mathrm{~cm}$ throat radius, with a structural throat radius of $2 \mathrm{~m}$. The $10 \mathrm{~cm}$ radius was chosen so that the throat cross sectional area power flux of $1.54 \cdot 10^{5} \mathrm{MW} / \mathrm{m}^{2}$ was comparable to proposed NTR designs $\left(1.16 \cdot 10^{5} \mathrm{MW} / \mathrm{m}^{2}\right)^{72}$ and within extrapolation of current cryogenic engines such as the Space Shuttle Main Engine $\left(5.14 \bullet 10^{4} \mathrm{MW} / \mathrm{m}^{2}\right){ }^{73}$ A $\beta=1$ condition was assumed to calculate the magnetic field strength and required current for throat and upstream reservoir magnet coils. A specific heat ratio $(\gamma)$ of $5 / 3$ was used for a monatomic gas of three degrees of freedom.

The reservoir conditions were solved using Equations (29) and (30), and Equations (27) and (28) using reservoir values $(\mathrm{M}$ is throat Mach number $\equiv 1)$. The $\mathrm{T}_{\text {res }}$ was $252 \mathrm{eV}$, an order of magnitude lower than that at the scrape off layer. The required reservoir number density was $1.35 \cdot 10^{22} / \mathrm{m}^{3}$, two orders of magnitude greater than that in the scrape off layer. 


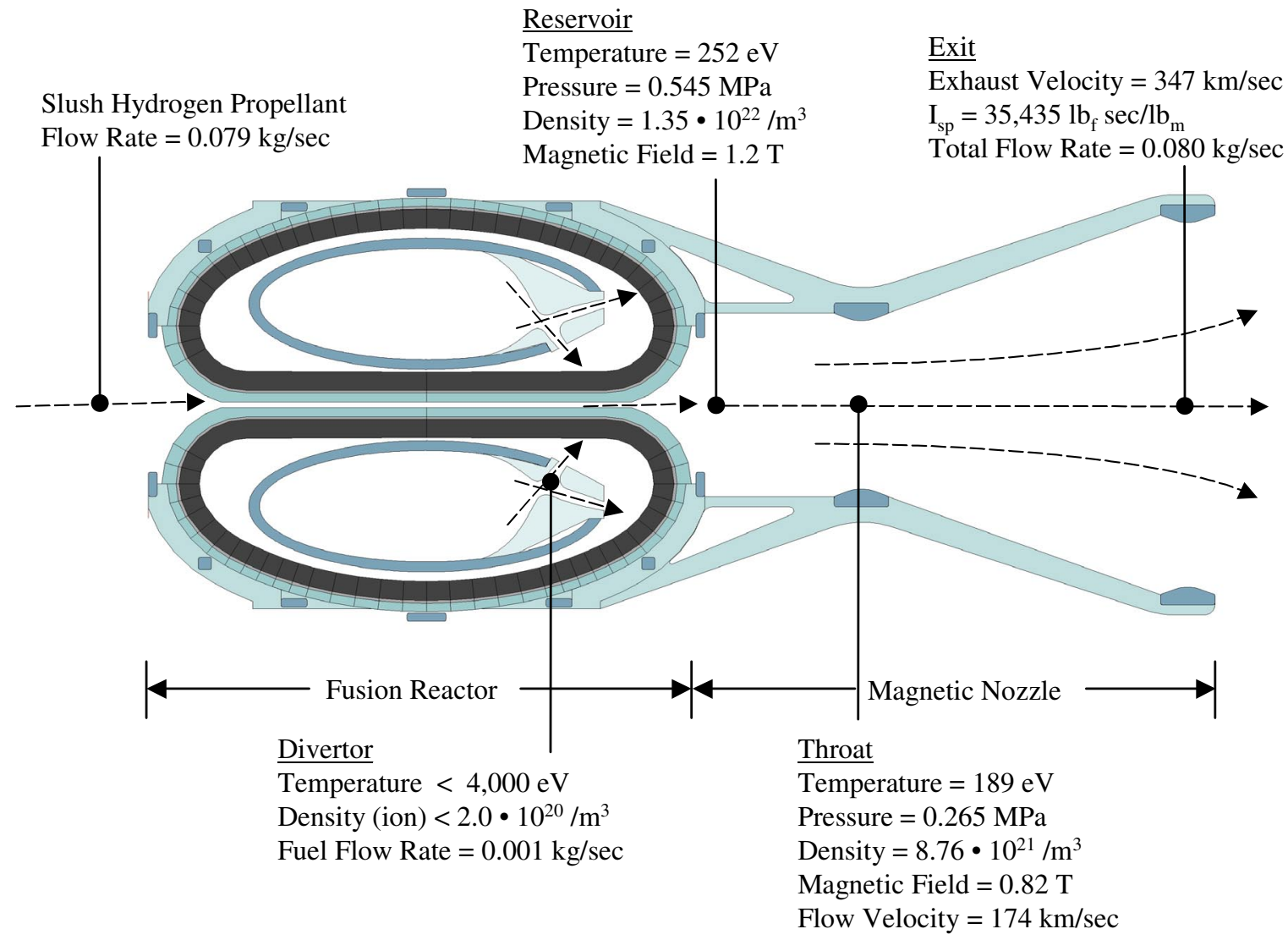

Figure 17: Plasma State Conditions Through Magnetic Nozzle

$$
\begin{gathered}
T_{\text {res }}=\left[1+\frac{\gamma-1}{2} M^{2}\right] T_{\text {throat }} \quad(M=1) \\
(P R)_{\text {res }}=\left[1+\frac{\gamma-1}{2} M^{2}\right]^{\frac{\gamma}{\gamma-1}}(P R)_{\text {throat }} \quad(M=1)
\end{gathered}
$$

The reservoir flow power $\left(\mathrm{P}_{\mathrm{res}}\right)$ and thermal velocity $\left(\mathrm{v}_{\text {res }}\right)$, which are related by the stagnation enthalpy $\left(\mathrm{h}_{\mathrm{res}}\right)$, can be estimated by Equations (31) and (32). The flow power can then be related to the available transport power $\left(\mathrm{P}_{\text {trans }}\right)$ from the reactor by Equation (33). Thus the jet power and state conditions throughout the nozzle can be correlated to the power delivered to the reservoir by the divertor (Figure 17). The $20 \%$ loss associated with the enthalpy transfer was assumed to exit the nozzle in the form of neutrals, ions with velocity vectors not strictly aligned with the thrust vector, collisions with support structure, line radiation, charge exchange, and other losses.

$$
\begin{gathered}
P_{\text {res }}=\dot{m}_{\text {total }} h_{\text {res }}=\dot{m}_{\text {total }} \frac{\gamma}{\gamma-1} R T_{\text {res }} \\
v_{\text {res }}=\sqrt{2 h_{\text {res }}} \\
P_{\text {res }}=\eta_{J} P_{\text {trans }}=\eta_{J} \frac{1}{2} \dot{m}_{\text {fuel }} v_{\text {fuel }}^{2}=\frac{1}{2} \dot{m}_{\text {total }} v_{\text {res }}^{2}
\end{gathered}
$$

The augmentation propellant mass flow rate

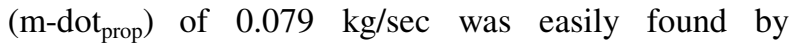

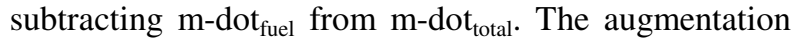
propellant was $\sim 78$ times that of the fuel, emphasizing that a significant portion of the magnetic nozzle system must be dedicated towards accommodating the injection of augmentation propellant. This great a mass infusion into the flow stream (compared to the relatively low flow rate from the reactor) may be great enough to adversely affect the plasma conditions back into the reactor. This could represent a significant operational problem and warrants future assessment. 


\section{REACTION CONTROL}

Positive control of a vehicle's attitude requires arresting various torques associated with natural perturbations as well as applying torques to achieve vehicle orientations and assist in steering. Most of the significant natural perturbations present in planetary orbit (such as gravity gradient-driven apsidal and nodal drifts, atmospheric drag, magnetic field interactions, etc.) are either significantly diminished or not applicable to interplanetary travel. The torque associated with solar radiation pressure was assessed due to the considerable surface area of the radiator. It was found that even the worst case orientation at Earth solar distance, the radiation torque was negligible.

Main propulsion steering assistance would, however, be needed. Though the single nozzle configuration would not be able to perform roll control, pitch and yaw was accomplished by selective, periodic reconfiguration of the nozzle's magnetic field geometry $^{74}$. This appeared to be the desirable way to steer the vehicle, given that the complex coupling between the magnetic nozzle and the reactor's divertor would make gimballing problematic. The method of operation, including the reconfiguration of individual coil currents to pitch/yaw steer, has yet to be defined.

A separate reaction control system (RCS) was added for roll control. (This system was also used for minor pitch and yaw attitude control when the main propulsion system was not operating, such as during planetary parking orbit or emergency re-start. Multiple, cross coupled firings would be required to null-out residual rates in this case.) Currently, RCS propulsion technology is transitioning from monopropellants to more advanced electric propulsion. Since the Discovery II was significantly more massive than today's spacecraft (requiring more impulse) and large quantities of electric power and hydrogen were available, the higher $\mathrm{I}_{\mathrm{sp}}$ hydrogen fueled arcjet was used for the RCS.

A vehicle system control model was not available to guide the design of the RCS from a "needs up", so a "top down" approach was used to determine how much control authority should exist for a reasonable impulse, power, propellant, and mass allocation. A design of a $8 \mathrm{MW} /$ thruster (i.e. two thrusters firing per couple, with one fore couple and one aft couple) yielded a thrust level of over $80 \mathrm{lb}_{\mathrm{f}}$ per thruster, with an impulse of $2,490 \mathrm{lb}_{\mathrm{f}}$ sec per thruster. The RCS thruster design was based on the NASA LeRC $30 \mathrm{~kW}$ hydrogen arcjet program. That program produced thrusters delivering $\mathrm{I}_{\mathrm{sp}}$ 's up to $1,460 \mathrm{lb}_{\mathrm{f}}$ $\mathrm{sec} / \mathrm{lb} \mathrm{m}$ and efficiencies of $30+\% .^{75}$ Using these values, mass and mass flow rates per thruster were extrapolated to be $2 \mathrm{mt}$ and $0.025 \mathrm{~kg} / \mathrm{sec}$ respectively. (Table 16)
Table 16: Reaction Control Characteristics

\begin{tabular}{|c|c|}
\hline Number of thruster units & 2 \\
\hline Number of thruster clusters per unit & 2 \\
\hline Number of thrusters per cluster & 2 \\
\hline Number of thrusters (total) & 8 \\
\hline Thruster type & arcjet \\
\hline Propellant & hydrogen \\
\hline Power per thruster (MW) & 8 \\
\hline Thrust $\left(1 b_{f}\right)$ & 83 \\
\hline Specific impulse $\left(\mathrm{lb}_{\mathrm{f}} \mathrm{sec} / \mathrm{lb} \mathrm{b}_{\mathrm{m}}\right)$ & 1,480 \\
\hline Mass flow rate $(\mathrm{kg} / \mathrm{sec})$ & 0.025 \\
\hline Efficiency $(\%)$ & 33.3 \\
\hline \multicolumn{2}{|l|}{ Roll maneuver } \\
\hline Angular velocity (deg/min) & 1.24 \\
\hline Torque $(\mathrm{N} \mathrm{m})$ & 2,200 \\
\hline Firing time (sec) & 30 \\
\hline Thrusters per maneuver & 4 \\
\hline Battery bank supplied energy (MJ) & 960 \\
\hline Number of start/stop rolls per day & 3 \\
\hline Total propellant mass $(\mathrm{mt})$ & 20 \\
\hline Total system dry mass ( 8 thrusters) (mt) & 16 \\
\hline
\end{tabular}

The RCS was composed of two units of four thrusters each. Each unit housed two thruster clusters $180^{\circ}$ apart. Each cluster contained two thrusters, oriented $180^{\circ}$ from the other. Full roll control was provided with both units firing simultaneously. The aft unit was mounted on the aft end of the truss network, forward of the reactor. The forward unit was adjacent to the avionics suite. (Initially the forward RCS unit was placed forward of the propellant tankage, since $~ 90 \%$ of the vehicle's fully loaded mass ( $~ 80 \%$ of its dry mass) was aft of that point.) This new location offered greater vehicle control due to greater lever arm. Assessment of the truss network's ability to withstand the resulting bending moments was deferred.

An angular velocity of $\sim 1 \%$ minute could be produced by four RCS thrusters firing about the minimum axis (roll) with a torque of $\sim 2,200 \mathrm{~N}-\mathrm{m}$ and a firing time of 30 seconds. The thrusters were powered by discharging the dual use battery bank, which was capable of supplying the $\sim 32 \mathrm{MW}$ input power to the four thrusters. The total propellant consumption, assuming three 30 second start/stop roll corrections per day, every day (for 1 year) was $\sim 6 \mathrm{mt}$. Pitch and yaw maneuvers performed by the magnetic nozzle during steady state interplanetary cruise were assumed to consume propellant at rates comparable to the RCS thrusters. The total amount of hydrogen for $6 \mathrm{D}$ reaction control was therefore $20 \mathrm{mt}$. The total mass of the 8 thruster RCS was $16 \mathrm{mt}$, assuming all power processing was performed by the battery bank system. 


\section{POWER CONVERSION}

The primary function of the power conversion system was to utilize some of the Bremsstrahlung and neutron radiation by "thermalizing" this energy flux so that a fraction could be converted into electrical output power. A closed Brayton thermodynamic cycle (referred to as a Closed Cycle Gas Turbine (CCGT)), was selected to accomplish the thermal-to-electric energy conversion. The CCGT was chosen on the basis of proven design and fabrication experience. In addition, CCGT systems have the advantage of a significant performance and reliability database and the ability to use an inert gas (gaseous helium (GHe)) as the working fluid. The GHe was used to transport heat energy (cooling the inboard assembly and TF/PF coils) at high temperature directly to the turbine.

The CCGT power system was modeled using the BRMAPS code developed at NASA GRC by one of the authors (Juhasz). The code's power flow design is illustrated in Figure 18. The total thermal power supplied to the CCGT conversion system was $96 \mathrm{MW}_{\mathrm{t}}$ at $1700{ }^{\circ} \mathrm{K}$. Of this total thermal power, the gas turbine power system converted 28.6 MW to electrical output power using a turbo-alternator. Alternator shaft-toelectrical/other power conversion losses totaling $\sim 2.5 \mathrm{MW}$ were included in the $\sim 67 \mathrm{MW}_{\mathrm{t}}$ rejected to space by flat plate heat pipe radiators that had a total radiating surface area of $10,000 \mathrm{~m}^{2}$. The conceptual design of the power conversion system was patterned after helium turbine designs for nuclear power plants advanced in Germany three decades ago. ${ }^{48,49}$ Some units were actually constructed at $\sim 25 \mathrm{MW}_{\text {e }}$ power levels. ${ }^{76}$

The salient design and performance parameters (BRMAPS inputs) for the power conversion system were: a peak temperature (turbine inlet) of $1700^{\circ} \mathrm{K}$, and pressure of $7.5 \mathrm{MPa}(74 \mathrm{~atm})$. The cycle temperature and pressure ratios of 3.5 and 4.95 respectively, yielded a cycle thermodynamic efficiency of $\sim 29.8 \%$. With the peak cycle pressure (i.e. compressor exit pressure) set at $74 \mathrm{~atm}$, the resultant inlet pressure was $14.95 \mathrm{~atm}$, and the total mass flow rate was $26.3 \mathrm{~kg} / \mathrm{sec}$ of He. Cycle state points are shown in Figure 18.

A flat plate radiator design was chosen that used lightweight carbon-carbon, parallel duct heat pipes. This radiator technology has been proposed as the appropriate match for closed Brayton cycle with a high temperature, gas reactor heat source. ${ }^{51}$ The radiator parameters were derived from successful fabrication and laboratory demonstration tests of a heat pipe radiator element. These tests confirmed a radiator specific mass of $1.5 \mathrm{~kg} / \mathrm{m}^{2}$ and suggested further improvement to $<1 \mathrm{~kg} / \mathrm{m}^{2}$ may be possible (based on radiation from both sides). Thus a radiator specific mass value of $1.0 \mathrm{~kg} / \mathrm{m}^{2}$ was assumed here. Other parameters for the power system radiators are shown in Table 17 and Figure 18.

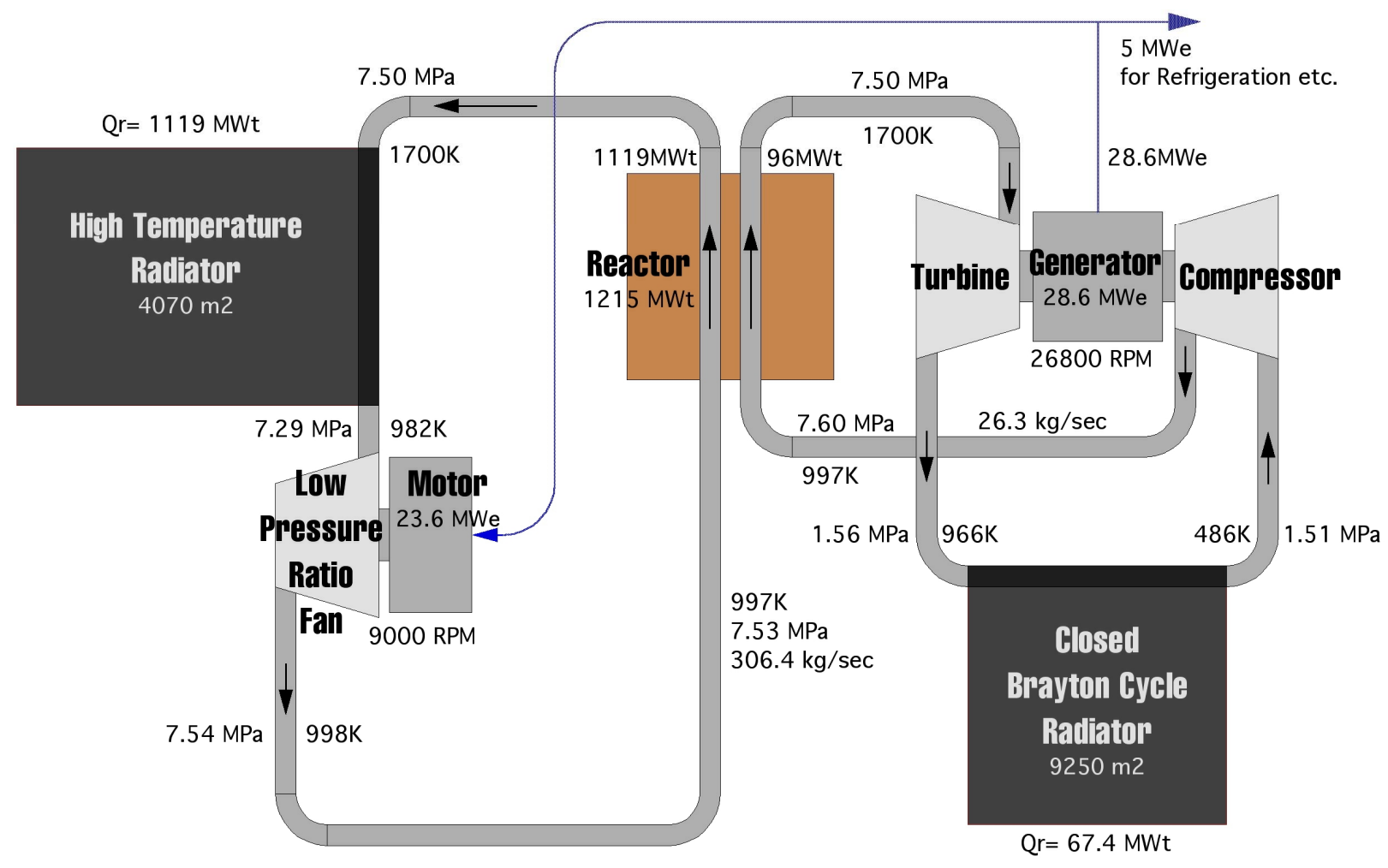

Figure 18: Closed Brayton Cycle Power Conversion and Reactor Coolant Systems 
Table 17: Other Power Conversion System Characteristics

\begin{tabular}{lc}
\hline Radiator type & Heat pipe \\
Radiator material & carbon - carbon \\
Radiator specific mass $\left(\mathrm{kg} / \mathrm{m}^{2}\right)$ & 1.0 \\
Emissivity & 0.9 \\
Turboshaft speed $(\mathrm{rpm})$ & 26,800 \\
Voltage $(\mathrm{kVAC})$ & 10 \\
AC frequency $(\mathrm{Hz})$ & 1,200 \\
\hline
\end{tabular}

GHe working fluid entered each radiator at $966^{\circ} \mathrm{K}$. Note that the input assumptions for the design of this radiator were based on the worst case spacecraftto-sun distance of $1 \mathrm{AU}$; maximum insolation angle of $+/-30^{\circ}$; radiator surface emissivity of 0.9 ; absorptivityto-emissivity ratio, $\alpha / \varepsilon$, of 0.2 ; fin effectiveness of 0.95 ; beginning-of-life excess area of $110 \%$. Thus the total radiating area was $9,250 \mathrm{~m}^{2}$ for the turboset, and $750 \mathrm{~m}^{2}$ for the power conditioning radiator (utilizing mediumhigh temperature electronics, capable of operating at $\left.480{ }^{\circ} \mathrm{K}\right)$. The combined rectangular panel two-sided radiator area was $10,000 \mathrm{~m}^{2}$. This area was arranged as twelve flat panels, rejecting heat from both sides. Each panel was $162 / 3 \mathrm{~m}$ wide, $25 \mathrm{~m}$ long (length limited by payload fairing, deployment, and rigidity constraints).

The turbine hub-to-tip ratios were set at 0.93 , yielding a turboset casing diameter of $0.8 \mathrm{~m}$ and turbine drive length (including the alternator-generator) of $\sim 3 \mathrm{~m}$. With the turbo shaft rotational speed of $26,800 \mathrm{rpm}$, the alternator was driven at synchronous speed to generate $1200 \mathrm{~Hz}$ - 3-phase AC power with a 4-pole alternator and design terminal voltage of $10 \mathrm{kVAC}$. Table 18 illustrates the power conversion system's mass properties, with a total mass of $30 \mathrm{mt}$.

Table 18: Power Conversion Mass Properties

\begin{tabular}{lr}
\hline Compressor & 3 \\
Turbine & 1 \\
Alternator & 3 \\
Management and distribution & 6 \\
Radiator & 9 \\
Power conditioning radiator & 1 \\
Support structure & 2 \\
Flywheel & 5 \\
---------------------- & -- \\
Total $(\mathrm{mt})$ & 30 \\
\hline
\end{tabular}

It was advantageous to generate most of the power at the voltage demanded by the largest loads. The power consumed by the GHe coolant loop motor/fan $\left(\sim 23.6 \mathrm{MW}_{\mathrm{e}}\right)$ represented over $80 \%$ of the total power output of the power conversion system. As a result, the power was generated in the appropriate form (voltage, amperage, and phase) for the motor in order to minimize heavy power conversion transformers and associated systems. Voltage transformers, however, were included in power management and distribution. To counteract gyroscopic torque on start-up, a highspeed $(60,000 \mathrm{rpm})$ flywheel momentum storage system was included. These systems controlled angular momentum and unbalanced torques associated with high-speed turbomachinery start-up, and prevented transfer of torsion loads into lightweight structure such as the central truss network. ${ }^{77}$

\section{HIGH HARMONIC FAST WAVE HEATING}

High Harmonic Fast Wave (HHFW) heating is a promising method of non-inductive plasma heating due to its potential for high heating efficiency (driven plasma current to injected power), high system efficiency (power output to power input), comparatively low system mass, and efficient volumetric packaging. The HHFW system was used for start-up and to provide some control of the plasma current radial profile, since the bootstrap current overdrive profile would not usually match the desired equilibrium profile. ${ }^{30}$ The overdriven profile regions would have to be nulled out, while the under-driven regions would have their currents augmented. Based partially on planning for the PPPL NSTX reactor ${ }^{30,78}$ and the ARIES series of conceptual designs, ${ }^{79,80}$ assessments of the operating process were made and power consumption/mass properties were estimated.

The HHFW system supplied power at high resonances of the ion cyclotron frequency. A HHFW system operating at 5 to $20^{\text {th }}$ harmonics of the ion cyclotron frequency should have excellent absorption within spherical torus-like plasmas in order to drive electrons and heat ions. ${ }^{30}$ Lower harmonics could be used to provide heating for the plasma core. Though not as mature as other technologies (i.e. neutral beams), the HHFW experience database continues to develop with the potential for an up to factor of ten efficiency improvement over today's systems. A projected heating efficiency of $0.1 \mathrm{~A}$ (driven)/W (injected) was used. ${ }^{30}$ The forecasted system efficiency used was comparable to that forecasted for neutral beam systems: $~ 70 \%$ (power out to wall plug). ${ }^{79}$ In addition, the HHFW system components were generally more amenable to reactor integration, where neutron-sensitive equipment could be placed behind reactor shielding by flexible transmission lines. Volumetric and orientation concerns were generally less demanding than neutral beams (with 
no long beam-lines at right angles to the reactor midplane requiring awkward integration).

An effective reduction in first wall reflectivity due to the wall surface occupied by the HHFW system was estimated to be $\sim 1 \% .^{79}$ In addition, synchrotron radiation could adversely impact HHFW antennas upon shine-through. A solution to both problems might be multiple Faraday strips designed to be preferentially transparent to HHFW but reflective of synchrotron radiation. ${ }^{79}$ Due to the relative proximity of the frequencies, this remained an issue for further study.

A start-up power of $108 \mathrm{MW}$ was required from the HHFW to affect the overdriven current of 10.8 MA. With $70 \%$ system efficiency, a peak value of 154 MW of electrical power had to be supplied to the HHFW. The large HHFW power requirement was the primary design driver for the start-up battery bank. Though the estimate of bootstrap current fraction (and thus required injection power) was reasonable, great leverage exists in this area for system optimization.

The mass of the HHFW system proper (antennas/shields, transmission lines, RF generators, etc.) was expected to be minimal, ${ }^{81}$ though a quantitative assessment could not be completed in time for this study. Some of the more massive power conditioning equipment was book-kept under the startup power system. The remainder was set at $5 \mathrm{mt}$.

\section{PROPELLANT CRYO-TANKAGE}

The slush hydrogen propellant cryo-tankage was based on a pre-existing conceptual design ${ }^{1}$, itself predicated on operational or previously designed conceptual liquid hydrogen propellant tanks. ${ }^{82}$ The large quantity of propellant needed for the vehicle rendered even the largest past, current, or proposed liquid hydrogen tank designs unsuitable. The largest liquid hydrogen tank ever built and flown is the hydrogen component of the Space Shuttle's External Tank (ET), a $27.5 \mathrm{ft}(8.4 \mathrm{~m})$ diameter, $96.7 \mathrm{ft}(29.5 \mathrm{~m})$ long structure which can accommodate $103 \mathrm{mt}$ of propellant. ${ }^{83}$ The Saturn V's S-II hydrogen tank, a $33 \mathrm{ft}(10 \mathrm{~m})$ diameter, $53 \mathrm{ft}(16 \mathrm{~m})$ long structure accommodated $70 \mathrm{mt}$ of propellant. ${ }^{84}$ In order to carry the significant quantity required for the Discovery II (861 mt), multiple tanks of an even greater capacity were needed. Therefore, a conceptual design was made of the largest tank that appeared reasonable on the grounds of experience, engineering judgment, and ground transportation concerns.

An existing conceptual design of liquid hydrogen tankage done in part by the co-authors ${ }^{1}$ and
Table 19: Slush Hydrogen Propellant Tankage Characteristics

\begin{tabular}{lc}
\hline Number of tanks & 4 \\
Delivery mode & HLLV (robotic) \\
Length (end to end) $(\mathrm{m})$ & 37 \\
Length (barrel section) $(\mathrm{m})$ & 30 \\
Diameter $(\mathrm{m})$ & 10 \\
Volume $\left(\mathrm{m}^{3}\right)$ & 2,715 \\
Pressure $(\mathrm{psi})$ & 49.7 \\
Ullage $(\%)$ & 2.5 \\
Tank material & $\mathrm{GrEp}$ \\
Yield strength (ksi) & 80 \\
Axial loading (maximum) $(\mathrm{g}$ 's) & 4 \\
Wall thickness (maximum) $(\mathrm{mm})$ & 6.1 \\
Micro-meteoroid shielding material & Aluminum \\
Multi-layer insulation $(\mathrm{cm})$ & 5 \\
Operating temperature $\left({ }^{\circ} \mathrm{K}\right)$ & 14 \\
Refrigerant & $\mathrm{GHe}$ \\
Refrigeration power $(\mathrm{kW})$ & 29.5 \\
Number of settling motors $\left(500 \mathrm{lb}_{\mathrm{f}}\right)$ & 2 \\
Number of attitude motors $\left(50 \mathrm{lb}_{\mathrm{f}}\right)$ & 24 \\
Flight performance reserve $(\%)$ & 1 \\
Residuals and loses $(\%)$ & 3 \\
Total tankable propellant $(\mathrm{mt})$ & 215 \\
Dry mass (mt) & 22 \\
\hline
\end{tabular}

based on an NTR vehicle concept, ${ }^{82}$ was adopted for slush hydrogen tankage. Support systems, net mass, and power required to accommodate slush hydrogen above and beyond those for liquid hydrogen were for the most part negligible. ${ }^{85}$ One concern, however, was allowing sufficient additional tank volume and strength to accommodate slush that would re-liquefy during launch through docking with the Discovery II. Due to its ascent dependency and lack of launch scenario definition, no design provisions were made for this concern at this time. Tankage pressure and wall thickness were designed, however, to accommodate a $4 \mathrm{~g}$ acceleration during Earth-to-orbit launch; thus were probably conservative compared to interplanetary cruise. The original NTR tankage design was for a human Mars mission. A graphite-epoxy (GrEp) IM7/977-2 composite hydrogen tank material was used to obtain considerable mass savings over advanced aluminum alloys. ${ }^{1}$ The $10 \mathrm{~m}$ diameter was maintained in order not to significantly impact manufacturing and ground transportation limits. The 3:1 aspect ratio (cylindrical barrel section length-to-diameter) yielded a total tank length of $37 \mathrm{~m}$. The total tankable slush hydrogen propellant was calculated to be $215 \mathrm{mt}$. (Table 19). 
Table 20: Propellant Tankage Dry Mass Properties (single tank)

Structure (barrels, domes, insulation, skirts, etc.) 16 Electrical, power, fluid systems (avionics, etc.) 2 Reaction control (thrusters, propellants, etc.) 2 Interface hardware (OMV, launch attachments)

Total (mt)

Table 20 lists subsystem masses for a single cryo-tank with a dry mass of $22 \mathrm{mt}$. The subsystems were based on and modified from three studies, all of which were based primarily on the two Centaur upper stage configurations flying on the then-current Atlas/Centaur and Titan IV/Centaur expendable launch vehicles. In the resulting tank design, over $46 \%$ of the total mass was attributed to the composite tank (upper and lower domes, plus barrel section), and $22 \%$ attributed to the $5 \mathrm{~cm}$ multi-layer cryo-insulation plus aluminum micro-meteoroid shielding (sized for Mars orbit "mean space temperatures" to approximate the range of Earth to Saturn environment). Other components/subsystems included skirts, feedlines, autogenous bleed, zero-g vent, purge, etc. A significant amount of mass and system complexity was required by considering the means for delivering the propellant to the departure orbit via an HLLV. The fully loaded propellant tankage must be launched with all the requisite ETO-related systems (avionics, telemetry, range safety, etc.). Upon separation from the HLLV, the cryo-tankage must be capable of controlling its own attitude, rendezvous, and dock with the Discovery II, thus requiring a reaction control system and interface hardware. This included two $500 \mathrm{lb}_{\mathrm{f}}$ thrusters for rendezvous/settling and twenty four $50 \mathrm{lb}_{\mathrm{f}}$ thrusters for attitude control. Launch vehicle interface hardware was derived from Orbital Maneuvering Vehicle study data and also estimated truss attach hardware.

Table 21: Propellant Tankage Wet Mass Properties (single tank)

\begin{tabular}{llrr}
\hline Propellant & & 215 \\
& main impulse & 207 & \\
& flight perf. reserve & 2 & \\
Stage Dry & residuals/losses & 6 & \\
Adapter & & & 22 \\
Contingency & (30\% of dry mass) & 8 \\
----------- & & ----- \\
GLOW (mt) & & 251 \\
\hline
\end{tabular}

Table 21 illustrates the mass summary for the fully loaded cryo-tankage in its ETO launch configuration (i.e., including adapter and associated contingency). Added to the useable, main impulse propellant was a flight performance reserve (FPR) of $1 \%$ (of main impulse propellant), consistent with past mission experience to accommodate in-flight dispersions. Estimates of residuals and chill-down losses were also included and made up 3\% (of the total tankable propellant). The adapter was sized to accommodate launch loads and was chargeable to the propellant tankage payload, although it would only be used from ETO and not retained by the Discovery II. A $30 \%$ weight growth allowance was assessed on the tankage dry mass and adapter. The gross liftoff weight (GLOW) of a fully loaded cryo-tankage payload was $251 \mathrm{mt}$, of which $207 \mathrm{mt}$ was slush hydrogen available for main impulse propulsion.

\section{REFRIGERATION}

Refrigeration was used to perform the initial cool down of these systems prior to reactor ignition, remove any residual reactor heat not transferred by the GHe coolant during steady state operation, and remove ambient (outer space) heating. Considerable extrapolation was required from current space-qualified, long life, mechanical cryocooler technology. ${ }^{86}$ These refrigeration systems are typically used for very small ( $\sim .1$ to $\sim 10$. W) heat loads, primarily IR sensors, low temperature optics, and other instrumentation. Published mass and power scalings were used despite the considerable extrapolations required, rather than applying large ground based systems that rely on utilizing the ambient terrestrial environment. A gaseous helium Stirling cycle system (an extrapolation from an MMS $4 \mathrm{~K}$ model $)^{86}$ with a $3^{\circ} \mathrm{K}$ low temperature sink $\left(T_{L}\right)$ was used to refrigerate the solid/liquid $\mathrm{D}^{3} \mathrm{He}$ fuel pellets. A gaseous helium Stirling cycle system (an extrapolation from the Ball Hybrid J-T/Stirling system $)^{86}$ with a $\mathrm{T}_{\mathrm{L}}$ of $10^{\circ} \mathrm{K}$ was used to refrigerate the slush hydrogen propellant. A liquid nitrogen, multistage Joule-Thomson (J-T) system (somewhat similar to a Ball liquid nitrogen-based COOLLAR 65/120 J-T system $)^{86}$ with a $\mathrm{T}_{\mathrm{L}}$ of $64.3^{\circ} \mathrm{K}$ was used to refrigerate the $65^{\circ} \mathrm{K}, \quad 25 \mathrm{TF} / \mathrm{PF} /$ divertor/magnetic nozzle superconducting coils and the central conductor.

To estimate refrigeration system electrical power and mass requirements, scaling laws derived from a variety of cryocoolers (though largely from Creare single and multi-stage reverse Brayton system units) were used. $^{87}$ (The inconsistency in the basis of scaling laws and the proposed systems is noted, but was deemed necessary given the paucity of appropriate data.) Multi-stage reverse Brayton systems appeared to have the lowest specific masses compared to Stirling 
systems, particularly at higher cooling capacities. ${ }^{86}$ An ambient heating temperature of $172^{\circ} \mathrm{K}$ was used (corresponding to average Mars orbit heat flux) to approximate an effective mission average, outer solar system thermal environment. With the tankage insulation and shielding described earlier, approximately $0.0725 \mathrm{~W} / \mathrm{m}^{2}$ was the average thermal power per (multilayer insulated) area to be removed (Q) from the propellant and fuel tanks using Equation 34 (where $\mathrm{C}_{1}=8.95 \mathrm{E}-08$ and $\mathrm{C}_{2}=5.39 \mathrm{E}-10$ ). ${ }^{87}$ The corresponding heat to be rejected was $519 \mathrm{~W}_{\mathrm{th}}$, and $13 \mathrm{~W}_{\text {th }}$ from the propellant and fuel tankage respectively, and was small compared to residual reactor steady state heating that reached the YBCO superconductor and its G-C substrate (Table 22). Radiation analysis (Equation 13) found that over $88 \mathrm{~kW}$ and $45 \mathrm{~kW}$ residual neutron heat was deposited in the YBCO central conductor and G-C substrate respectively, and represented almost all of the refrigeration requirement.

$$
\begin{gathered}
Q=\left[C_{1} \bar{N}^{2.56}\left(T_{H}^{2}-T_{C}^{2}\right) / 2+C_{2} \varepsilon\left(T_{H}^{4.67}-T_{C}^{4.67}\right)\right] \\
*(f / N)
\end{gathered}
$$

The electrical power $\left(\mathrm{P}_{\text {refrig }}\right)$ required to operate the refrigeration systems was calculated by Equation (35), where the heat rejection temperature $\left(\mathrm{T}_{\mathrm{H}}\right)$ was chosen to be $350{ }^{\circ} \mathrm{K}$, sufficiently greater than the ambient outer solar system background. The coefficient of performance (COP) for the refrigerators was set at $15 \%$ of the Carnot COP, where current technology values of $10 \%$ are typical and predicted near term efficiencies are $\sim 20 \%{ }^{87} 3,950 \mathrm{kWe}$ of electrical power was required to refrigerate the superconducting coils. The significantly smaller propellant and fuel tankage requirements were $118 \mathrm{kWe}$ and $10 \mathrm{kWe}$ respectively (Table 22).

$$
\begin{gathered}
P_{\text {refrig }}=\frac{Q}{0.15}\left[\frac{T_{H}-T_{L}}{T_{L}}\right] \\
M_{\text {refrig }}=0.9 \bullet 171.85 T_{L}^{-0.85} Q^{0.52}
\end{gathered}
$$

The masses of the refrigeration $\left(\mathrm{M}_{\text {refrig }}\right)$ systems were determined from Equation (36) and included all subsystems except power source and radiator ${ }^{87}$. Since the additional radiator mass was assumed to be small compared to the main array, the total refrigeration power (operation and rejected heat) to be rejected was just added to the main array. The masses of the
Table 22: Refrigeration Characteristics

\begin{tabular}{lccc}
\hline Refrigeration loop & $\mathrm{TF} / \mathrm{PF}$ & $\mathrm{SLH}_{2}$ & $\mathrm{D}^{3} \mathrm{He}$ \\
\hline Cycle & $\mathrm{J}-\mathrm{T}$ & Stirling & Stirling \\
Refrigerant & $\mathrm{LN}_{2}$ & $\mathrm{GHe}$ & $\mathrm{GHe}$ \\
COP $(\%$ Carnot) & 15 & same & same \\
Temperature (hot) $\left({ }^{\circ} \mathrm{K}\right)$ & 350 & same & same \\
Temperature (cold) $\left({ }^{\circ} \mathrm{K}\right)$ & 64.3 & 10 & 3 \\
Temperature (wall) $\left({ }^{\circ} \mathrm{K}\right)$ & 65 & 14 & 4 \\
Heat transferred $\left(\mathrm{W}_{\mathrm{th}}\right)$ & 133,350 & 519 & 13 \\
Power required $\left(\mathrm{kW}_{\mathrm{e}}\right)$ & 3,950 & 118 & 10 \\
Mass $(\mathrm{mt})$ & 1.66 & 0.45 & 0.19 \\
\hline
\end{tabular}

superconducting coils, propellant tankage, and fuel tankage refrigeration systems were $1.66 \mathrm{mt}, 0.45 \mathrm{mt}$, and $0.19 \mathrm{mt}$ respectively (Table 22).

\section{FUEL INJECTOR AND TANKAGE}

The preponderance of fuel injector design, fabrication, and operation experience has been with ${ }^{2} \mathrm{D}$, and DT fuelling. Two stage, light gas gun devices have accelerated $1-3 \mathrm{~mm}$ frozen ${ }^{2} \mathrm{D}$ pellets up to $2.5 \mathrm{~km} / \mathrm{sec}^{88,89}$ Alternate concepts such as electrothermal guns have been tested with plastic (Lexan polycarbonate) pellets to similar velocities. ${ }^{90}$ The gross dimensions and masses for most concepts were small compared to other major systems $(<1 \mathrm{~m}$ lengths and $<1 \mathrm{mt}$ masses). ${ }^{91,92}$

Very little effort, however, has be devoted to issues pertaining to injection of frozen $\mathrm{D}$ pellets encapsulating (liquid) ${ }^{3} \mathrm{He}$. It is thought that injecting such pellets during ignited, high temperature operation will be very difficult. ${ }^{25}$ An earlier conceptual design study of a mirror reactor propulsion concept suggested a two-stage mechanical (centrifugal) and electromagnetic railgun injector be used for $\mathrm{D}^{3} \mathrm{He}$ pellets. ${ }^{93}$ This study and others have suggested a potential approach involving frozen light metal shelled (lithium deuteride) pellets encapsulating ${ }^{3} \mathrm{He}$ fluid. ${ }^{25,93}$ Estimated final velocities between 10 and $30 \mathrm{~km} / \mathrm{sec}$ with acceleration lengths limited to up to $20 \mathrm{~m}$ forced one study to use light metal shelling to mitigate barrel heating and stress on the pellet. The light metal shelling did have a detrimental affect $\left(17 \%\right.$ loss in jet power) ${ }^{93}$ on reactor performance even at small $(<6 \%)$ pellet mass fraction. Pellet evaporation is not thought to be a major potential contributor to fuel depolarization. ${ }^{25}$

The pellet injector design for the Discovery II was based on a promising concept for large pellets and high velocities: a plasma armature electromagnetic railgun. These devices have demonstrated accelerating $1 \mathrm{~cm}$-sized polycarbonate pellets up to $\sim 7 \mathrm{~km} / \mathrm{sec}$, with 
energy transfer efficiencies of $\sim 10 \%$ and constant accelerations up to $\sim 4.5 \cdot 10^{6} \mathrm{~g}$ 's. ${ }^{94,95}$ They are, however, not re-useable at these demanding levels due to significant performance-driven erosion of the bore. Also, acceleration of frozen $D$ pellets has been to only half these velocities due to wall and electrode erosion, not particle disassembly. ${ }^{94}$ No pre-accelerator was used.

Table 23: Fuel Injector Characteristics

\begin{tabular}{lc}
\hline Injector type & EM railgun \\
Length $(\mathrm{m})$ & 185 \\
Bore height $(\mathrm{cm})$ & 3 \\
Power (steady state) $(\mathrm{kWe})$ & 507.5 \\
Fueling rate (steady state) $(\mathrm{Hz})$ & 1 \\
Pellet acceleration $(\mathrm{g}$ 's) & 27,580 \\
Final velocity $(\mathrm{km} / \mathrm{sec})$ & 10 \\
Energy transfer efficiency $(\%)$ & 10 \\
Injector mass (mt) & 5 \\
Power (start-up) $(\mathrm{kWe})$ & 101.5 \\
Fueling rate (start-up) $(\mathrm{Hz})$ & 0.2 \\
Pellet material & solid D, liquid ${ }^{3} \mathrm{He}$ \\
Pellet length (cube) $(\mathrm{cm})$ & 2.2 \\
Pellet mass $(\mathrm{g})$ & 1.015 \\
\hline
\end{tabular}

The pellet injector for the Discovery II operated at only $1 \%$ of acceleration levels of today's experimental devices; thus a frequency capability of 1 $\mathrm{Hz}$ was assumed. A square bore of height $3 \mathrm{~cm}$ was used for the cubic pellets that were $2.2 \mathrm{~cm}$ on a side. The pellets were $\sim 1 \mathrm{~g}$ each and were not pre-accelerated. A final pellet speed of $10 \mathrm{~km} / \mathrm{sec}$ was assumed to be required for adequate penetration to the

Table 24: Fuel Tankage Characteristics

\begin{tabular}{lc}
\hline Fuel tank length $(\mathrm{m})$ & 4.1 \\
Fuel tank diameter $(\mathrm{m})$ & 6 \\
Tank material & $\mathrm{GrEp}$ \\
Yield strength (ksi) & 80 \\
Axial loading (maximum) (g's) & 4 \\
Wall thickness (maximum) (mm) & 6.1 \\
Micro-meteoroid shielding material & Aluminum \\
Multi-layer insulation $(\mathrm{cm})$ & 5 \\
Operating temperature $\left({ }^{\circ} \mathrm{K}\right)$ & 4 \\
Refrigerant & $\mathrm{GHe}$ \\
Refrigeration power $(\mathrm{kWe})$ & 10.2 \\
Fractional burn up $(\%)$ & 2.2 \\
Flight performance reserve $(\%)$ & 1 \\
Residuals and loses $(\%)$ & 3 \\
Total tankable fuel $(\mathrm{mt})$ & 11 \\
Dry mass (mt) & $\sim 1$ \\
\hline
\end{tabular}

plasma core. The ample central truss length was thought to permit a sufficiently low pellet acceleration so as not to require light metal shelling. Using almost the entire length of the available central truss $(185 \mathrm{~m})$, a constant acceleration of 27,580 g's was required. The steady state power needed (assuming today's energy conversion efficiency of $10 \%$ ) was $507.5 \mathrm{kWe}$. Given the paucity of experimental hardware for this regime of operation and type of pellet, it was hypothesized that such an electromagnetic railgun injector would be no greater than $5 \mathrm{mt}$ in mass (including electrodes, magnets, arc initiator, etc.) (Table 23).

$$
f_{b}=\left[1+\frac{2}{n \tau<\sigma v>}\right]^{-1}
$$

To sustain a total fusion power of 7,895 MW, $\mathrm{D}^{3} \mathrm{He}$ fuel with a specific energy of $3.52 \cdot 10^{14} \mathrm{~J} / \mathrm{kg}$ must be consumed at a rate of $\sim 22 \mathrm{mg} / \mathrm{sec}$. The fraction of fuel burn up $\left(\mathrm{f}_{\mathrm{b}}\right)$ was calculated by integrating Equation (37) throughout the reactor core, where $\langle\sigma v\rangle$ is the Maxwellian averaged fusion reactivity for the $\mathrm{D}^{3} \mathrm{He}$ fusion reaction. The average $\mathrm{D}^{3} \mathrm{He}$ burn up fraction was calculated to be only $\sim 2.2 \%$, requiring a fuel mass flow rate $\left(\mathrm{m}^{-\mathrm{dot}_{\text {fuel }}}\right.$ ) of $\sim 1 \mathrm{~g} / \mathrm{sec}$. At this consumption rate, a 118 day trip to Jupiter required $11 \mathrm{mt}$ of $\mathrm{D}^{3} \mathrm{He}(\sim 6.7 \mathrm{mt}$ of ${ }^{3} \mathrm{He}$ ). (Note that $\sim 18 \mathrm{~kg} / \mathrm{yr}$ represents a rough estimate of the potential U.S. annual production capability of ${ }^{3} \mathrm{He} .{ }^{96}$ ) Tankage mass for the solid/liquid $\mathrm{D}^{3} \mathrm{He}$ fuel pellets was estimated by scaling from the slush hydrogen propellant tankage. For the Jupiter mission, the cylindrical fuel tank had a $3 \mathrm{~m}$ radius, $4.1 \mathrm{~m}$ length, and wet mass (including reserves, residuals, losses) of $1.5 \mathrm{mt}$. (Table 24)

\section{STARTUP/RE-START REACTOR AND BATTERY}

The startup system consisted of a $2 \mathrm{MW}_{\mathrm{e}}$, pellet bed nuclear fission reactor power system and a nickel hydrogen $(\mathrm{NiH})$ bipolar battery bank. Weeks prior to departure, the startup reactor was used to gradually refrigerate the $\mathrm{TF} / \mathrm{PF} /$ divertor/magnetic nozzle coils, initiate and ramp up their current, provide auxiliary power, and charge-up the battery. When all systems and crew were ready, RF heating initiated plasma formation in the core of the fusion reactor. Then the final plasma startup sequence was executed with a $\sim 6.5$ to $10 \mathrm{sec}$ battery discharge providing $\sim 1 \mathrm{GJ}$ of energy (154 $\mathrm{MW}_{\mathrm{e}}$ of input power) to the $\mathrm{HHFW}$ system. Together with fuel pellet injection (at $0.2 \mathrm{~Hz}$ for start-up), the reactor passed through breakeven and proceeded to ignition conditions.

Prior to current startup and HHFW heating to ignition conditions, $\sim 1 \mathrm{MW}$ of auxiliary RF heating at the electron cyclotron frequency was used to create a small volume of high conductivity plasma $\left(\mathrm{T}_{\mathrm{e}} \sim 100 \mathrm{eV}\right.$ 
and $\left.\mathrm{n}_{\mathrm{e}} \sim 10^{19} / \mathrm{m}^{3}\right)$. This plasma was outboard of the major radius and assisted in the current startup process. ${ }^{97}$ This plasma conditioning, referred to as preheating, permitted a small radius $\left(\mathrm{a}_{0} \sim 0.2\right.$ to $\left.0.4 \mathrm{~m}\right)$ current channel to be established with a relatively low initial loop voltage $(<25 \mathrm{~V}$ as opposed to $\sim 100-200 \mathrm{~V}$ without RF assist). With the onset of current initiation and establishment of the desired safety factor $q$ in the small current channel, the startup major radius near the outboard midplane was gradually shifted inward to $\mathrm{R}=2.48 \mathrm{~m}$. During this "expanding radius startup", 98 new layers of plasma were added to the warm core through ionization of a regulated gas feed. Major radius compression permitted minor radius expansion and a simultaneous increase in plasma current while a constant q was maintained. As the plasma minor radius grew in size, sufficiently high levels of current and plasma density were achieved to ensure adequate confinement of energetic protons from $\mathrm{D}^{3} \mathrm{He}$ fusion. Heating to ignition conditions could then commence.

A small fission reactor was the heat source for a dynamic energy conversion system that supplied power during startup and emergency re-start of the fusion reactor/propulsion system. During steady state, interplanetary cruise, the fission reactor power system continued to supply auxiliary power, primarily for fuel injection, RCS roll, battery re-charge, and other miscellaneous operations. A high temperature $\left(1700{ }^{\circ} \mathrm{K}\right)$ gas cooled reactor (HTGR) of the pellet bed type $^{99}$ with a thermal power of $\sim 6 \mathrm{MW}_{\mathrm{t}}$ was coupled to a regenerated (85\% effectiveness) "Closed Cycle Gas Turbine" CCGT power system. With a net thermal-toelectric conversion efficiency of $>35 \%$, it provided $2 \mathrm{MW}_{\mathrm{e}}$ of electrical power at an alternator terminal voltage of $5 \mathrm{kV}$. The reactor heated $\sim 2.1 \mathrm{~kg} / \mathrm{sec}$ of GHe flow to $1,700{ }^{\circ} \mathrm{K}$ in order to drive the gas turbine. An instrument rated shield was included, designed for a neutron fluence of $10^{15} \mathrm{n} / \mathrm{cm}^{2}$ and a gamma dose rate of 1 mega-rad at a $40 \mathrm{~m}$ separation distance over a period of 5 years. Owing to the high thermal power density of the HTGR $\left(>1 \mathrm{~kW}_{\mathrm{t}} / \mathrm{cm}^{3}\right)$ it was possible to enclose the entire 3 liter reactor volume, external to the cylindrical pressure vessel, with an additional $15 \mathrm{~cm}$ thick tungsten $4 \pi$ shield, resulting in an added shield mass of $\sim 1.4 \mathrm{mt}$. The $4 \pi$ shield reduced radiation exposure to safe levels should reactor subsystem service by crew be needed, as well as protection for equipment located adjacent to it. A flat plate heat pipe radiator of similar design as the main radiator provided the radiator total two-sided surface area of $706 \mathrm{~m}^{2}$. Note that the total surface area of the radiator is $10 \%$ larger at the beginning of the mission than required. Thus a loss of $10 \%$ of the initial heat pipe area due to micrometeoroid impacts can be absorbed without decreasing electric power output. The maximum operating pressure for this system was $\sim 4$ atm., casing diameters were $0.7 \mathrm{~m}$, and the total drive length was $3 \mathrm{~m}$. The optimum design speed for the turbo-alternator was $36,000 \mathrm{rpm}$. For electric power output, a directly driven 2-pole alternator generating 3-phase AC current at $600 \mathrm{~Hz}$ was used. The total system mass was calculated to be $\sim 10 \mathrm{mt}$, a third of which represented radiation shielding (Table 25).

\section{Table 25: Startup Fission Reactor and Power System Characteristics}

\begin{tabular}{lc}
\hline Reactor type & HTGR pellet bed \\
Thermal power $\left(\mathrm{MW}_{\mathrm{th}}\right)$ & 6 \\
Reactor volume $(\mathrm{l})$ & 3 \\
Radiation shielding $(\mathrm{n}, \gamma)$ (solid angle) & $4 \pi$ \\
Shielding material & Tungsten \\
Power cycle type & Regen CCGT \\
Electrical power $\left(\mathrm{MW}_{\mathrm{e}}\right)$ & 2 \\
Working fluid & $\mathrm{GHe}$ \\
Fluid temperature $(\mathrm{hot})\left({ }^{\circ} \mathrm{K}\right)$ & 1,700 \\
Fluid flow rate $(\mathrm{kg} / \mathrm{sec})$ & 2.1 \\
Cycle temperature ratio & 3.6 \\
Cycle pressure ratio & 2.5 \\
Power conversion efficiency $(\%)$ & 35.6 \\
Turbo-alternator speed (rpm) & 36,000 \\
AC frequency $(\mathrm{Hz})$ & 600 \\
Drive length $(\mathrm{m})$ & 3 \\
System diameter $(\mathrm{m})$ & 0.7 \\
Radiator surface area (two sided) $\left(\mathrm{m}^{2}\right)$ & 706 \\
Total system mass $(\mathrm{mt})$ & 10 \\
\hline
\end{tabular}

The $\mathrm{NiH}$ bipolar batteries were derivatives of devices designed and tested at NASA GRC. These $82 \%$ efficient, high peak power systems were capable of specific energies of $180 \mathrm{~kJ} / \mathrm{kg}$ and energy packaging densities of over $80 \mathrm{Whr} / \mathrm{liter}^{100,101}$ For the required startup energy pulse, a bank of volume $3.36 \mathrm{~m}^{3}$ with a mass of $5.6 \mathrm{mt}$ would be needed (Table 26).

Table 26: Battery Bank Characteristics

\begin{tabular}{lc}
\hline Battery type & $\mathrm{NiH}_{2}$ bipolar \\
Specific energy $(\mathrm{kJ} / \mathrm{kg})$ & 180 \\
Energy packing density $(\mathrm{W} \mathrm{hr} / \mathrm{l})$ & 82.6 \\
Discharge power (maximum) $(\mathrm{GW})$ & 1 \\
Discharge time $(\mathrm{sec})$ & $6.5-10$ \\
Power conversion efficiency $(\%)$ & 82 \\
Volume $\left(\mathrm{m}^{3}\right)$ & 3.36 \\
Mass $(\mathrm{mt})$ & 5.6 \\
\hline
\end{tabular}

Should the fusion reactor need to be re-started during the interplanetary transit, the same startup sequence would be followed. The startup reactor power would also be used to maintain the refrigeration of the $\mathrm{TF} / \mathrm{PF} /$ divertor/magnetic nozzle coils (to prevent them 
from "going normal") and propellant/fuel tankage, to provide crew accommodations, communications, and maintain other auxiliary systems. Should the battery bank fail to re-start the reactor, sufficient startup reactor power would be available to re-charge the bank in two hours for another attempt while maintaining power to the other essential systems.

\section{AVIONICS/COMMUNICATIONS}

The concept vehicle's avionics suite would be composed of primary/backup computers, guidance, navigation, and control system (GN\&C), tracking, data display, sequencing, and instrumentation. The exponential rate of growth in speed and capability of electronics and computer technology will no doubt enable future systems to be vastly superior to today's systems at only a fraction of the mass and power required. Thus minimal attention was devoted towards trying to extrapolate avionics system capabilities and requirements. A sufficient avionics suite of the future should be available with a total mass $<1 \mathrm{mt}$ with arbitrarily small power consumption.

The communication system was presumed to be a derivative of the recently developed Ka band (20 to $30 \mathrm{GHz}$ ) technology. This NASA GRC digital processing and storage communication technology is currently capable of integrated data, voice, and video with typical throughput rates in excess of $10^{8} \mathrm{bits} / \mathrm{sec}$ and up to $10^{10}$ bits $/ \mathrm{sec}^{102,103,104}$ Two $5 \mathrm{~m}$ diameter deployable $\mathrm{Ka}$ band dish antennas were used for the audio, video, and data communication system. Dual antennas were used to enable simultaneous two-way communications with the departing and arriving planets (and redundancy if necessary). Mounted on $12 \mathrm{~m}$ truss booms aft of the crew habitat payload, the dish antennas were positioned so that the rotating crew payload would not obstruct both simultaneously. Heating from the radiators was not addressed.

The total avionics/communication system mass was set at $2 \mathrm{mt}$. The power for the avionics suite was assumed to be comparable to the Space Shuttle orbiter available power ${ }^{105} 0.02 \mathrm{MW}$. The power required by the communication antennas was indeterminate, since antenna diameters and powers at the destinations were undefined. Should ground stations comparable to the Deep Space Network be used, the power and size requirements could be further reduced. Therefore, a communication power was set at $0.2 \mathrm{MW}$.

\section{WEIGHT GROWTH CONTINGENCY}

Weight growth contingency is the margin allocated to compensate for the inevitable growth in mass experienced by aerospace systems as designs mature and construction proceeds. Underestimates become apparent and technical problems are solved by incorporating solutions requiring additional mass. All new launch vehicle development programs carry such an allocation, though the percentage allowable varies as a function of component maturity. Experience with the development of eighteen major aerospace vehicles has demonstrated that from the point of initial contract proposal through acquisition of first unit, the total average weight growth experienced by military aerospace vehicles has been $25.5 \% .{ }^{106}$ For more than a two dozen major NASA manned and unmanned vehicles and spacecraft, from the point of phase C/D to first vehicle flight, most programs have experienced a similar $15 \%$ to $30 \%$ weight growth. ${ }^{107,108}$ Given the immaturity of the majority of technologies used on the Discovery II, past aerospace experience suggests a prudent minimum value of $30 \%$ weight growth allowance be assessed on the total dry mass of the propulsion system and the crew payload. Thus, weight growth contingencies of $149 \mathrm{mt}$ and $40 \mathrm{mt}$ were carried on the propulsion and payload systems respectively, representing significant mass property contributions.

\section{COMPARISON OF 2001: A SPACE ODYSSEY'S DISCOVERY vs. NASA's DISCOVERY II}

The motion picture epic 2001: A Space Odyssey and its accompanying novel are perhaps the most prominent $20^{\text {th }}$ century classics to have influenced the public's perception of "the beauty and complexity of space travel in the $21^{\text {st }}$ century" 109 . Director Stanley Kubrick's and science fiction author Arthur C. Clarke's focus on technical accuracy drove much of the behind the scenes work with advise by technical consultants from NASA and the aerospace industry. Clarke's experience in engineering complemented Kubrick's creativity and produced a story that validated the saying, 'good science fiction must first be good science'. A few thoughts on the 'good science' (good engineering) of their vehicle design effort, and how they compare with this NASA concept, are in order.

The mission of the Discovery spacecraft in 2001 was a Jupiter rendezvous in '.. a better part of a year" ${ }^{110}$. (The novel departed from the movie's plot in that the ultimate destination was Saturn via a gravity assist maneuver at Jupiter.) Though the propulsion system technology was not discussed in the movie, and only hinted at in the novel ('low-thrust plasma drive'), ${ }^{111}$ published accounts by Dr. Frederick Ordway indicated that his design for Discovery's propulsion system was based on a specific type of gas core nuclear fission technology (referred to as a "Cavradyne engine"). ${ }^{112}$ Gas core is a very advanced propulsion technology with an estimated capability somewhere 
between that of solid core nuclear fission and magnetic confinement nuclear fusion. Systems studies have estimated that gas core should be capable of $\mathrm{I}_{\mathrm{sp}}$ 's of $\sim 4,000$ to 6,000 seconds. ${ }^{113}$ This technology, however, would not operate via continuous thrust. A gas core engine would operate at high thrust $\left(\sim 10,000\right.$ 's $\left.\mathrm{lb}_{\mathrm{f}}\right)$ and is expected to have very long, but nonetheless discrete, burns of the order of 24 to 60 hours. ${ }^{113}$ Results elsewhere concluded that a gas core propulsion system operating between 2,000 and 5,000 seconds of $\mathrm{I}_{\mathrm{sp}}$ could perform a Jupiter rendezvous between 5 and 10 months respectively. ${ }^{114}$ It is therefore conceivable that a gas core-propelled spacecraft like Discovery could have accomplished the Jupiter mission with the trip time alluded to in the motion picture. Discovery II's trip times were shorter as would be expected with more advanced propulsion technology: 4 months to Jupiter and 7 months to Saturn, as discussed earlier. This stemmed from the greater specific power for this particular fusion vehicle concept, though as was mentioned, the $\alpha$ of Discovery was not known.

The overall size and layout of the vehicles were similar. The Discovery was $213 \mathrm{~m}$ (700 ft) long vs. the Discovery II's 240 m. Discovery's propulsion system appeared to be anything but modular and readily transportable by a HLLV however. Instead, it seemed to be the product of complex on-orbit assembly, rather than Discovery II's LEO docking after HLLV transport. Also, the generally assumed-to-be propellant tankage along Discovery's central spine appeared small by comparison to the cryo-tankage on Discovery II. It is also known that Clarke realized the need for a considerable expanse of radiators, but could not find a design that was aesthetically pleasing to the professional filmmakers. The radiators were eventually dropped altogether. On Discovery II, as with most nuclear-based propulsion concepts, radiators were a (large and heavy) indispensable part of the system.

The crew payload onboard Discovery was a $38 \mathrm{ft}$ diameter (5.8 $\mathrm{m}$ radius) sphere, containing an internal centrifuge. Ordway notes that he was aware of the need for a larger diameter to mitigate detrimental effects induced by Coriolis forces generated by short rotation arms. But the physical limits of the soundstage (and funds) dictated the eventual diameter of the centrifuge that became one of the better-known aspects of the movie. ${ }^{112}$ The $17 \mathrm{~m}$ radius used on Discovery II was more consistent with current technical opinions as was discussed earlier. With that greater radius, launching and assembling a spherical pressure vessel, or even a 'wheel', became unwieldy. This was why the Lab/Hab module with connecting tunnel concept was adopted. Thus Discovery II's artificial gravity design was considerably more conservative, particularly in terms of gravity gradient where its radial dimension was three times Discovery's and only $1 / 5$ of its gravity.

A considerable amount of engineering thought went into Discovery's crew accommodations. Ordway and his team performed thousands of man-hours designing layouts including health monitoring, flight instrumentation, storage, entertainment, medical, hibernation, gymnasium, food preparation, storm shelter, and other systems for the crew. ${ }^{112}$ The effort expended on these particular systems for Discovery II, by contrast, paled in comparison and was limited to merely scalings from current NASA design studies.

All things considered, the amount of credible engineering design that was used in 2001: A Space Odyssey's Discovery vehicle was remarkable. Though a different propulsion technology, it could be said that the effort by Ordway, Kubrick, Clarke, and the rest of the 2001 team to design a vehicle and a story that was 'good science' as well as 'good science fiction' appears in retrospect to have been successful.

\section{FUTURE AREAS OF ANALYSIS}

Due to time constraints, several potentially superior design solutions for various systems were not pursued. Further study will be performed and possibly incorporated into a future upgrade of the Discovery II. Future areas of analysis include: inclusion of preliminary results of the magnetic nozzle simulation and experiment data, examination of new single null open divertor concept, inclusion of CHE analysis, the creation of a ${ }^{3} \mathrm{He}$ recovery system, incorporation of a lower aspect ratio fusion reactor, and the redesign of the battery bank.

\section{CONCLUSIONS}

A conceptual vehicle system design predicated on a small aspect ratio spherical torus nuclear fusion reactor has the potential for enabling relatively fast outer solar system travel. The requirements for human missions to Jupiter (Saturn) were satisfied with a $172 \mathrm{mt}$ payload mass, a 1,690 (1,699) mt IMLEO, and 118 (212) day one way trip time. The vehicle concept provided high thrust $\left(4,000\right.$ to $\left.6,000 \mathrm{lb}_{\mathrm{f}}\right)$, high $\mathrm{I}_{\mathrm{sp}}$ $\left(35,000\right.$ to $\left.47,000 \mathrm{lb}_{\mathrm{f}} \mathrm{sec} / \mathrm{lb}_{\mathrm{m}}\right)$, and radial interplanetary transfers due to its high thrust-to-weight ( milli-g) capability. In situ refueling capability was required for all round trip missions. High orbit space basing was assumed. Design decisions were driven by the desire to maintain a balance between today's experimentally demonstrated technology and reasonable extrapolations to what might be available some 30 years from now. 
A complete vehicle concept was produced with analysis to a sufficient level to make certain assessments on general concept viability and research requirements. Systems analysis, design, and assessment was performed on an shielded/artificial gravity crew payload, central truss, nuclear fusion reactor, magnetic nozzle, power conversion (turbine, generator, radiator, conditioning), HHFW heating, refrigeration, tankage, avionics, startup fission reactor and battery bank, fuel pellet injection, communications, reaction control systems, mission design, assembly, and space operations. Detailed assessment was performed on reactor operations, including plasma characteristics, power balance/utilization, first wall, TF coils, radiation, and heat transfer. Overall feasibility of nuclear fusion propulsion systems must include assessments of related space infrastructure such as heavy lift launch vehicles, space based orbit transfer vehicles, and in situ resource utilization with associated cost of their operations.

An experiment is currently underway at Ohio State University to explore critical plasma conditions and flow behavior associated with very high power magnetic nozzles. Related theory development pertaining to plasma instabilities is also underway at Los Alamos National Lab. Another related experiment at Princeton Plasma Physics Lab's National Spherical Torus Experiment is under study.

Critical areas of research upon which the feasibility of this concept rests include: the demonstration of ignited, long term steady state, spinpolarized $\mathrm{D}^{3} \mathrm{He}$ fueled nuclear fusion reactor operation; the successful incorporation of a single null, open divertor capable of transferring large quantities of transport power to a direct propulsion system; the determination whether high density downstream propellant infusion adversely impacts fusion reactor operation; the identification of operations incompatible with spin polarized fuel; the assessment of feasibility of Coaxial Helicity Ejection; the fabrication of monolithic, high current, higher temperature $\left(65^{\circ} \mathrm{K}\right)$, superconductors; construction of high frequency-high velocity $\mathrm{D}^{3} \mathrm{He}$ pellet injection; development of ISRU infrastructure (particularly ${ }^{3} \mathrm{He}$ mining); and the successful demonstration of a high thrust, high efficiency magnetic nozzle. Once such technical issues are explored in depth, a judgment can be rendered as to the practicality of a solar system-class, nuclear fusionbased transportation system for the $21^{\text {st }}$ century.

\section{REFERENCES}

${ }^{1}$ Williams, C.H., "Trajectory Design and Mission Analysis of Fast, Outer Solar System Travel", AAS paper 01-161, February 2001.

${ }^{2}$ Williams, C.H. et al., "A Spherical Torus Nuclear Fusion Reactor Space Propulsion Vehicle Concept for Fast Interplanetary Piloted and Robotic Missions", AIAA paper 99-2704, June 1999.

${ }^{3}$ Williams, C.H. et al., “A Spherical Torus Nuclear Fusion Reactor Space Propulsion Vehicle Concept for Fast Interplanetary Travel", NASA TM-208831, NASA Lewis Research Center, Cleveland, OH, December 1998.

${ }^{4}$ Williams, C.H. and Borowski, S.K., "An Assessment of Space Propulsion Fusion Concepts and Desired Operating Parameters for Fast Solar System Travel", AIAA paper 97-3074, July 1997.

${ }^{5}$ Williams, C.H. and Borowski, S.K., "CommerciallyDriven Human Interplanetary Propulsion Systems: Rationale, Concept, Technology, and Performance Requirements", paper \#58, $13^{\text {th }}$ Symposium on Space Nuclear Power and Propulsion, Albuquerque, NM, January 1996.

${ }^{6}$ Williams, C.H., "An Analytic Approximation to Very High Specific Impulse and Specific Power Interplanetary Space Mission Analysis", NASA TM107058, NASA Lewis Research Center, Cleveland, OH, September 1995.

${ }^{7}$ Borowski, S.K., "A Comparison of Fusion/Antiproton Propulsion Systems for Interplanetary Travel", AIAA paper 87-1814, July 1987.

${ }^{8}$ Williams, S.N., "An Introduction to the Use of VARITOP," Jet Propulsion Lab, JPL doc. D-11475, January 1994.

${ }^{9}$ George, J., NASA Lewis Research Center, personal communication, June 1997.

${ }^{10}$ Hyde, R.A., “A Laser-Fusion Rocket for Interplanetary Propulsion", $34^{\text {th }} \quad$ International Astronautical Federation, Budapest, Hungary, October 1983. 
${ }^{11}$ Isakowitz, S.J., "International Reference Guide to Space Launch Systems”, AIAA, 1991.

${ }^{12}$ Borowski, S.K. et al., "Nuclear Thermal Rocket/Vehicle Design Options for Future NASA Missions to the Moon and Mars", NASA TM-107071, September 1995.

${ }^{13}$ Kulcinski, G.L., "Importance of Helium-3 for the Future", Report of the NASA Lunar Energy Enterprise Case Study Task Force, NASA TM-101652, July 1989.

${ }^{14}$ Parkinson, R.C., "Project Daedalus: Propellant Acquisition Techniques", Journal of the British Interplanetary Society, supplement, 1978.

${ }^{15}$ Clark, B.C., Lockheed Martin Corp., personal communication, May 2001.

${ }^{16}$ Borowski, S.K., et al., "Artificial Gravity Vehicle Design Option for NASA's Human Mars Mission Using 'Bimodal' NTR Propulsion", AIAA paper 99-2545, June 1999.

${ }^{17}$ Paloski, W.H. et al, "Artificial Gravity Workshop," Proceedings and Recommendations, January 1999.

${ }^{18}$ Clark, B.C., "Concept 6: An Artificial Gravity Mars Spaceship", Space Technology, vol. 11, no. 4, 1991.

${ }^{19}$ Wilson, J.W., et al., "Shielding Strategies for Human Space Exploration", NASA Conference Publication 3360, December 1997.

${ }^{20}$ Stanford, M., and Jones, J.A., "Space Radiation Concerns for Manned Exploration", Acta Astronautica, Vol. 45, No. 1, 1999.

${ }^{21}$ Howe, S.D., "Nuclear Rocket to Mars", Aerospace America, August 2000.

${ }^{22}$ Watson, J., "Design Considerations for an Astronaut Monorail System for Large Space Structures and the Structural Characterization of its Positioning Arm", masters thesis (engineering mechanics), Old Dominion University, August 1992.

${ }^{23}$ Borowski, S.K. and Strickler, D.J., "A ProfileAveraged, Steady State Model for Studying Ignited- and Driven-Tokamak Operations", unpublished ORNL paper, 1985.
${ }^{24}$ Hale, Gerald, Los Alamos National Lab, personal communication, February 1998.

${ }^{25}$ Mitarai, O., et al., "Spin Polarization Effect on Ignition Access Condition for D-T and $\mathrm{D}^{3} \mathrm{He}$ Tokamak Fusion Reactors", Fusion Technology, July 1992.

${ }^{26}$ Christiansen, J.P., et al., "Global Energy Confinement H-Mode Database for ITER", Nuclear Fusion, vol. 32, no. 2, 1992.

${ }^{27}$ Kaye, S.M., et al., "ITER L Mode Confinement Database", Nuclear Fusion, vol. 37, no. 9, 1997.

${ }^{28}$ McCool, S.C., et al., "A Mega-Ampere Spherical Tokamak Design for Beta-Limit and Confinement Studies", Transactions of Fusion Technology, vol. 27, April 1995.

${ }^{29}$ Grisham, L., Princeton Plasma Physics Lab, personal communication, March 1998.

${ }^{30}$ Peng, M., Princeton Plasma Physics Lab, email, May 1999.

${ }^{31}$ Stambaugh, R.D. et al., "The Spherical Torus Approach to Magnetic Fusion Development", Fusion Technology, vol. 30, December 1996.

${ }^{32}$ Anonymous brochure, "Beryllium Optical Materials", Brush Wellman Inc., undated.

${ }^{33}$ Dombrowski, D.E. et al., "Thermomechanical Properties of Beryllium", Atomic and Plasma-Material Interaction Data for Fusion, vol. 5, IAEA TR-1182, February 1995.

${ }^{34}$ Anonymous brochure, "Designing With Beryllium", Brush Wellman, Inc., 1997.

${ }^{35}$ Krajcik, R.A., "The Effect of a Metallic Reflector Upon Cyclotron Radiation", Nuclear Fusion, vol. 13, 1973.

${ }^{36}$ McAllister, L.E., Engineered Materials Handbook: Composites, Vol. 1, ASM International Pub., 1987.

${ }^{37}$ Ulrickson, M., Sandia National Lab, personal communication, March 1999.

${ }^{38}$ Dombrowski, D.E., Brush Wellman Inc., personal communication, March 1999. 
${ }^{39}$ Santarius, J., University of Wisconsin, personal communication, March 1999.

${ }^{40}$ Longhurst, G., Idaho National Engineering Lab, personal communication, March 1999.

${ }^{41}$ LaMarsh, J.R., Introduction to Nuclear Engineering, Addison-Wesley Pub., 1977.

${ }^{42}$ Hughes, D.J., et al., Neutron Cross Sections, BNL325, vol. 5, July 1955.

${ }^{43}$ Lide, D.R., CRC Handbook of Chemistry and Physics, 81st edition, CRC Press, 2000-2001.

${ }^{44}$ Powell, J., et al., "DEALS: a Demountable Externally Anchored Low Stress Superconducting Magnet System for Fusion Reactors", BNL-50616, January 1977.

${ }^{45}$ Service, R.F., "New Superconductor Stands Up to Magnetic Fields”, Science, May 1995.

${ }^{46}$ Awaji, S., et al., "Crossover from Intrinsic to Extrinsic Pinning for $\mathrm{YBa}_{2} \mathrm{Cu}_{3} \mathrm{O}_{7}$ Films”, Cryogenics, vol. 39, 1999.

${ }^{47}$ Puzniak, R., et al., "Enhancement of Critical Current Density in Fast Neutron Irradiated Melt-Textured $\mathrm{YBa}_{2} \mathrm{Cu}_{3} \mathrm{O}_{7-\mathrm{x}}$, Cryogenics, vol. 33, No. 3, 1993.

${ }^{48}$ Wacenovsky, M., et al., "Influence of Fast Neutron Irradiation on Critical Currents and Irreversibility Lines in MPMG-Processed $\mathrm{YBa}_{2} \mathrm{Cu}_{3} \mathrm{O}_{7}$ Superconductors", Cryogenics, vol. 33, No. 1, 1993.

${ }^{49}$ Tipler, P.A., Physics, Worth Pub., 1976.

${ }^{50}$ Moses, R.W. and Young, W.C., "Analytic Expressions for Magnetic Forces on Sectored Toroidal Coils", Proc. Eng. Probl. Fusion Res., 6 ${ }^{\text {th }}$ IEEE, 1976.

${ }^{51}$ Teller, E., editor, Fusion, vol.1, Academic Press Pub., 1981.

${ }^{52}$ Boyer, R., et al., editors, "Materials Properties Handbook: Titanium Alloys", ASM International Pub, 1994.

${ }^{53}$ Manual of Steel Construction Allowable Stress Design, $9^{\text {th }}$ edition, American Institute of Steel Construction, September 1989.
${ }^{54}$ Devoto, R.S. et al., "Projections for a Steady State Tokamak Reactor Based on ITER", UCID 21519, December 1989.

${ }^{55}$ Roth, J.R., Introduction to Fusion Energy, Ibis Pub., 1988.

${ }^{56}$ Baker, C.C., et al., "Technology Implications of Advanced Fusion Fuel Cycles", Proc. International Conf. On Plasma Science, IEEE, 1979.

${ }^{57}$ Kreith, F., Principles of Heat Transfer, Harper \& Row Pub., 1973.

${ }^{58}$ Post, D. et al., "The ITER Power and Particle Control System", Fusion Technology, December 1996.

${ }^{59}$ Araki, M., et al., "Analytical Study of Electromagnetic Forces Induced By Eddy Current in ITER Divertor", Fusion Technology, December 1996.

${ }^{60}$ Sevier, D.L. et al., "Implications of Steady State Operation on Divertor Design", Fusion Technology, December 1996.

${ }^{61}$ Green, L., Westinghouse Science and Technology Center, personal communication, May 1998.

${ }^{62}$ Turchi, P.J., Ohio State University, and Peng, M., Princeton Plasma Physics Lab, personal communication, June 1999.

${ }^{63}$ Gerwin, R., "Summary Report on Plasma PropellantMagnetic Nozzle Interface", DOE preprint, Los Alamos National Lab, April 1999.

${ }^{64}$ Cikanek, H.A., "Lewis Research Center Revised Submissions to the Advanced Technology Lead Center for the Fiscal Year 2000 Budget Call", NASA official letter, June 1998.

${ }^{65}$ Turchi, P.J., et al., "Gigawatt, Quasi-Steady Plasma Flow Facility for Fusion Rocket Simulations", AIAA paper 98-3592, July 1998.

${ }^{66}$ Turchi, P.J., et al., "Gigawatt, Quasi-Steady Plasma Flow Facility for Fusion Rocket Simulations", AIAA paper 98-3592, July 1999. 
${ }^{67}$ Mikellides, I.G., et al., "Theoretical Design of a Magnetic-Nozzle Acceleration System for Fusion Propulsion", AIAA paper 2000-3367, July 2000.

${ }^{68}$ Gerwin, R.A., et al., "Characterization of Plasma Flow Through Magnetic Nozzles", Astronautics Lab (AFSC) AL-TR-89-092, final report, February 1990.

${ }^{69}$ Kamhawi, H., Ohio State University, unpublished notes, April 1997.

${ }^{70}$ Turchi, P.J., Ohio State University, unpublished notes, April 1997.

${ }^{71}$ Turchi, P.J., Ohio State University, email, June 1998.

${ }^{72}$ Borowski, S.K. and Dudzinski, L.A., "“2001: A Space Odyssey' Revisited - The Feasibility of 24 Hour Commuter Flights to the Moon Using NTR Propulsion with LUNOX Afterburners", AIAA paper 97-2956, July 1997.

${ }^{73}$ Sutton, G.P. and Ross D.M., Rocket Propulsion Elements, John Wiley \& Sons Pub., 1976.

${ }^{74}$ Turchi, P.J., Ohio State University, personal communication, June 1998.

${ }^{75}$ Sankovic, J.M., et al., "Hydrogen Arcjet Technology", NASA TM-105340, October 1991.

${ }^{76}$ Boehm, E., "Gas Turbines for Nuclear Power Plants", off-print for Euratom Review, February 1968.

${ }^{77}$ Dolce, J.L., “A Power Management and Distribution Concept for Electrically-Propelled Interplanetary Vehicles", NASA GRC presentation, February 2001.

${ }^{78}$ Spitzer, J. et al., "Engineering Design of the National Spherical Tokamak Experiment", Fusion Technology, vol. 30, December 1996.

${ }^{79}$ Mau, T.K. et al., "The Radio-Frequency CurrentDrive System for the ARIES-I Tokamak Power Reactor", Fusion Engineering and Design, vol. 24, 1994.

${ }^{80}$ Mau, T.K. et al., "Current Drive Studies for the ARIES Steady State Tokamak Reactors", Fusion Engineering and Design, vol. 25, 1994.
${ }^{81}$ Swain, D.W., Oak Ridge National Lab, e-mail, May 1999.

82، Nuclear Reactor/Propulsion System Interaction", General Dynamics Space Systems Division, final report, November 1993.

83، "Space Shuttle System Summary", Rockwell International Space Systems Group, May 1980.

84"Saturn V News Reference", NASA Marshall Space Flight Center, et al., December 1968.

${ }^{85}$ Kudlac, M., NASA Lewis Research Center, personal communication, June 1998.

${ }^{86}$ Glaister, D.S., et al., "An Assessment of the State of Cryocooler Technology for Space Applications", AIAA paper 98-5203, 1998.

${ }^{87}$ Kittel, P., et al., "Cryocoolers for Human and Robotic Missions to Mars”, Cryocoolers 10, 1999.

${ }^{88}$ Frattolillo, A., et al., "Development of a Two-Stage Pneumatic Repeating Pellet Injector for the Refueling of Long-Pulse Magnetic Confinement Fusion Devices", Fusion Technology, December 1997.

${ }^{89}$ Combs, S.K., et al., "Pellet Injector Research and Development at ORNL”, Fusion Technology, 1994.

${ }^{90}$ Kincaid, R.W. and Bourham, M.A., "Electrothermal Plasma Gun as a Pellet Injector", Fusion Technology, 1994.

${ }^{91}$ Bourham, M.A., North Caroline State University, personal communication, June 1998.

${ }^{92}$ Milora, S.L., Oak Ridge National Lab, personal communication, June 1998.

${ }^{93}$ Carpenter S.A. and Deveny, M.E., "Mirror Fusion Propulsion System (MFPS): An Option for the Space Exploration Initiative (SEI)", Space Technology, vol. 14, No. 1, Elsevier Science Ltd. Pub., 1994.

${ }^{94}$ Drobyshevski, E.M., et al., "Electromagnetic Launch of MM-Size Pellets to Great Velocities", Fusion Technology, Vol. 26, November 1994. 
${ }^{95}$ Hawke, R.S., et al., "Summary of EM Launcher Experiments Performed at LLNL", IEEE Transactions on Magnetics, Vol. MAG-22, No. 6, November 1986.

${ }^{96}$ Wittenberg, L.J., et al., "Lunar Source of ${ }^{3} \mathrm{He}$ for Commercial Fusion Power", Fusion Technology, September 1986.

${ }^{97}$ Borowski, S.K., Peng, Y-K. M., and Kammash, T., "Radio-Frequency-Assisted Current Startup in the Fusion Engineering Device," Fusion Technology, Vol. 6, July 1984.

${ }^{98}$ Borowski, S.K., "Inductive Current Startup in Large Tokamaks With Expanding Minor Radius and RF Assist," Oak Ridge National Laboratory Report FEDC83/8, February 1984.

${ }^{99}$ Juhasz, A.J., El-Genk, M.S., and Harper, W., "Closed Brayton Cycle Power System with a High Temperature Pellet Bed Reactor Heat Source for Nuclear-Electric Propulsion Applications", NASA TM-105933, October 1992.

${ }^{100}$ Cataldo, R.L., "Test Results of a Ten Cell Bipolar Nickel-Hydrogen Battery", NASA TM-83384, August 1983.

${ }^{101}$ Cataldo, R.L., NASA Lewis Research Center, personal communication, June 1998.

102،Advanced Communication Technology Satellite", NASA Fact Sheet, ACTS Pub \#301.

${ }^{103}$ Cook, D. and Bauer, B., “Advanced Communications Technology Satellite Program Overview”, April 1993.

${ }^{104}$ Kunath, R., NASA Lewis Research Center, personal communication, June 1998.

105“'Space Shuttle System Summary”, Rockwell International, May 1980.

${ }^{106}$ Hawkins, K., "Space Vehicle and Associated Subsystem Weight Growth", SAWE paper 1816, May 1988.

${ }^{107}$ Anonymous presentation, "MSFC Programs Weight Growth History", April 1979.
${ }^{108}$ Smith R.E. et al., "Weight Control and Weight Histories of Marshall Space Flight Center Projects", NASA MSFC-RPT-1553 A, July 1991.

${ }^{109}$ TV Guide, TV Guide Magazine Group, N.Y., N.Y., date unknown.

${ }^{110}$ S. Kubrick, 2001:A Space Odyssey, Metro-GoldwynMayer, Borehamwood, England,1968.

${ }^{111}$ A.C. Clarke, 2001: A Space Odyssey, New American Library, New York, 1968.

${ }^{112}$ F.I. Ordway, "2001: A Space Odyssey”, Spaceflight, Vol. 12, No. 3, Mar. 1970, pp. 110-117.

${ }^{113}$ R.G. Ragsdale, "To Mars in 30 Days by Gas-Core Nuclear Rocket", Astronautics and Aeronautics, Jan. 1972, pp. 65-71.

${ }^{114}$ W.E. Moeckel, "Comparison of Advanced Propulsion Concepts for Deep Space Exploration", Journal of Spacecraft, Vol. 9, No. 12, Dec. 1972, pp. 863-868. 


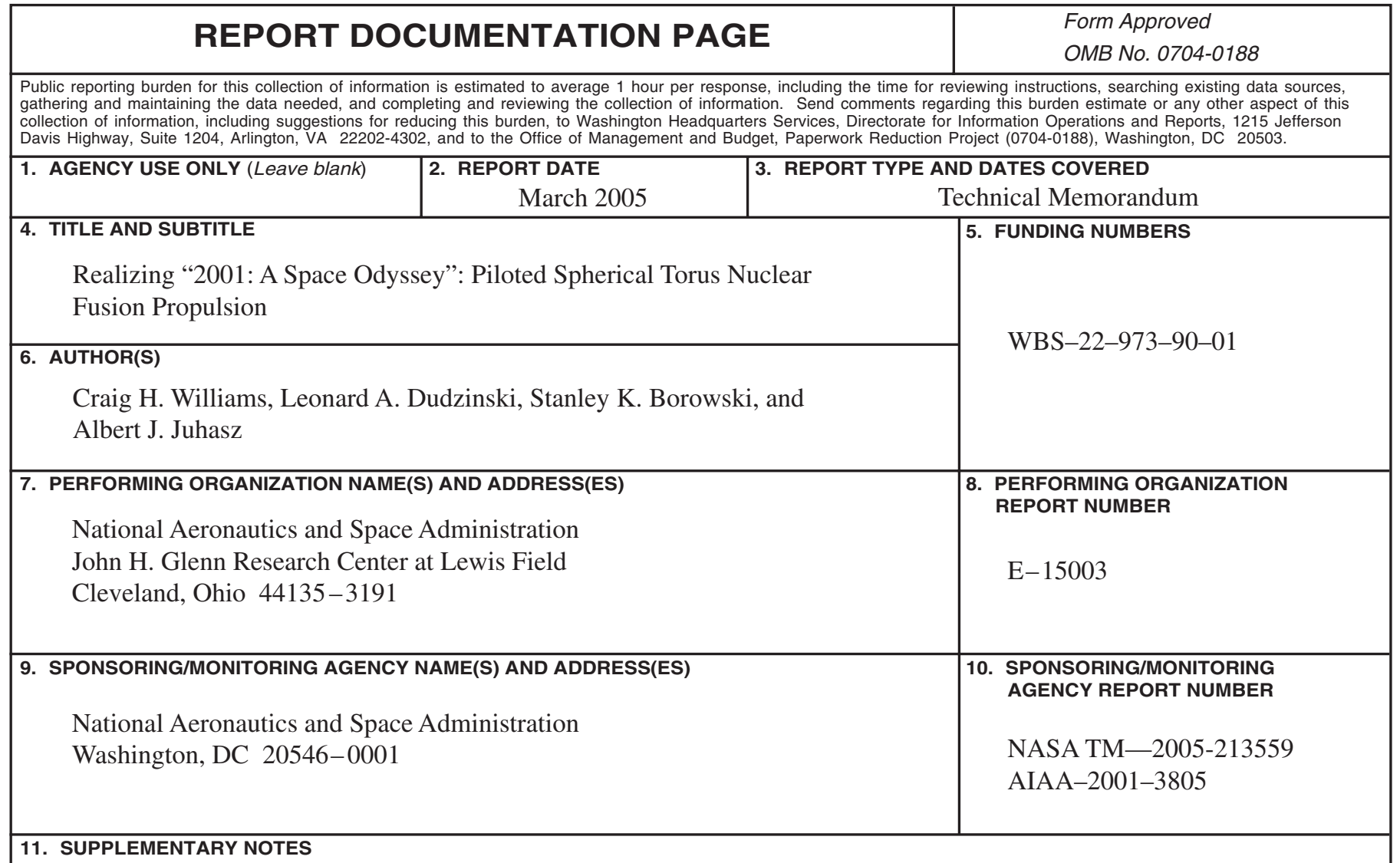

Prepared for the 37th Joint Propulsion Conference and Exhibit cosponsored by the AIAA, SAE, AIChE, and ASME, Salt Lake City, Utah, July 8-11, 2001. Responsible person, Craig H. Williams, organization code PBM, 216-977-7063.

\begin{tabular}{|l|l}
\hline 12a. DISTRIBUTION/AVAILABILITY STATEMENT & 12b. DISTRIBUTION CODE
\end{tabular}

Unclassified - Unlimited

Subject Category: 15

Available electronically at http://gltrs.grc.nasa.gov

This publication is available from the NASA Center for AeroSpace Information, 301-621-0390.

13. ABSTRACT (Maximum 200 words)

A conceptual vehicle design enabling fast, piloted outer solar system travel was created predicated on a small aspect ratio spherical torus nuclear fusion reactor. The initial requirements were satisfied by the vehicle concept, which could deliver a $172 \mathrm{mt}$ crew payload from Earth to Jupiter rendezvous in 118 days, with an initial mass in low Earth orbit of 1,690 mt. Engineering conceptual design, analysis, and assessment was performed on all major systems including artificial gravity payload, central truss, nuclear fusion reactor, power conversion, magnetic nozzle, fast wave plasma heating, tankage, fuel pellet injector, startup/re-start fission reactor and battery bank, refrigeration, reaction control, communications, mission design, and space operations. Detailed fusion reactor design included analysis of plasma characteristics, power balance/ utilization, first wall, toroidal field coils, heat transfer, and neutron/x-ray radiation. Technical comparisons are made between the vehicle concept and the interplanetary spacecraft depicted in the motion picture 2001: A Space Odyssey.

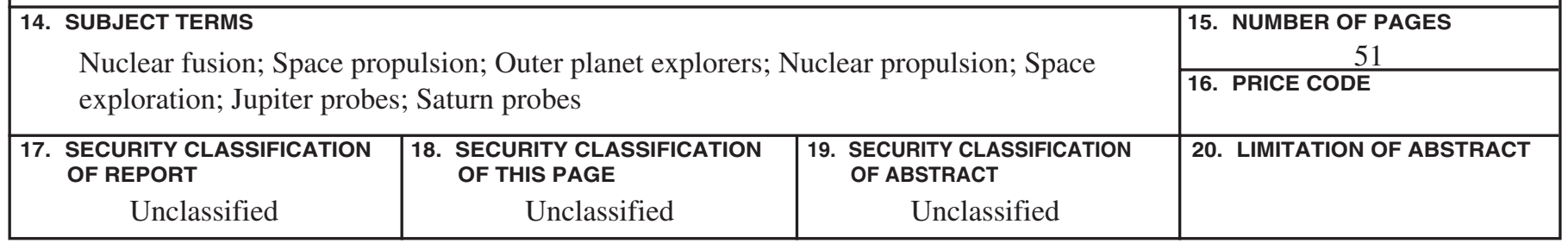



